\title{
45. NEOGENE PLANKTONIC FORAMINIFERS FROM THE WESTERN TROPICAL INDIAN OCEAN, LEG $115^{1}$
}

\author{
Edith Vincent ${ }^{2}$ and Monique Toumarkine ${ }^{3}$
}

\section{INTRODUCTION}

Neogene calcareous sediments were recovered at 12 sites in the western Indian Ocean (Table 1). Approximately $100010-\mathrm{cm}^{3}$ samples, at a sampling interval of about $1.5 \mathrm{~m}$, were collected from one hole at each site for planktonic foraminifer analysis. Samples were washed through a $63-\mu \mathrm{m}$ mesh sieve. Dried samples were weighted before and after washing to obtain the percentage of sand-size components, which are given in Table 2. The distribution of selected planktonic key species in each hole is reported in Tables 3-14, where the occurrence of a species in a sample is indicated with a cross ( $\mathrm{x})$ and a slash sign $(/)$ indicates that this species is extremely rare or presents some taxonomic ambiguity.

A visual estimate of the abundance of various components other than planktonic foraminifers is also given in Tables 3-14, with the abbreviations R (rare), F (few), C (common), and A (abundant). An estimate of the foraminiferal fauna preservation state is reported at the right of each table using a qualitative preservation scale as follows: G (good), M (moderately good), P (poor), and VP (very poor). Also reported are the abundances of planktonic foraminifer fragments and foraminifers embedded in a calcareous matrix, both of which are a function of the preservation state (the first reflecting the intensity of carbonate dissolution and the second, the occurrence of recrystallization).

Table 15 summarizes the biostratigraphic zonal assignments.

An overview of planktonic foraminifer distribution in Neogene sediments from Ocean Drilling Program (ODP) Leg 115 has been presented in Backman, Duncan, et al. (1988) based on a preliminary examination of core-catcher samples. From the more detailed investigations conducted in this study, only tables and species illustrations are presented in this report because of publication deadlines. Taxonomic and biostratigraphic discussions will be presented elsewhere.

\section{ACKNOWLEDGMENTS}

Funding for this study was provided by INSU/CNRS under DBT and IST programs. This is Contribution CNRS-INSUDBT No. 127. We thank E. Masure for her friendly help and expertise in obtaining scanning electron microscope photographs.

\section{REFERENCES}

Backman, J., Duncan, R. A., et al., 1988. Proc. ODP, Init. Repts., 115 College Station, TX (Ocean Drilling Program).

Barron, J. A., Keller, G., and Dunn, D. A., 1985a. A multiple microfossil biochronology for the Miocene. In Kennett, J. P. (Ed.), The Miocene Ocean: Paleoceanography and Biogeography. Mem. Geol. Soc. Am., 163:21-36.

\footnotetext{
${ }^{1}$ Duncan, R. A., Backman, J., Peterson, L. C., et al., 1990. Proc. ODP, Sci. Results, 115: College Station, TX (Ocean Drilling Program).

2 Département de Géologie Dynamique, Université Pierre et Marie Curie, Paris, France.

${ }^{3}$ Institut Géologique Albert de Lapparent, 21 rue d'Assas, 75006 Paris, France.
}

Barron, J. A., Nigrini, C. A., Pujos, A., Saito, T., Theyer, F., Thomas, E., and Weinreich, N., 1985b. Synthesis of biostratigraphy, central equatorial Pacific, Deep Sea Drilling Project Leg 85: refinement of Oligocene to Quaternary biochronology. In Mayer, L., Theyer, F., Thomas, E., et al., Init. Repts. DSDP, 85: Washington (U.S. Govt. Printing Office), 905-934.

Berggren, W. A., Kent, D. V., and Van Couvering, J. A., 1985. The Neogene: Part 2. Neogene geochronology and chronostratigraphy. In Snelling, N. J. (Ed), The Chronology of the Geological Record. Mem. Geol. Soc. (London), 211-260.

Blow, W. H., 1969, Late middle Eocene to recent planktonic foraminiferal biostratigraphy. In Brönnimann, P., and Renz, H. H. (Eds.), First International Conference on Planktonic Microfossils, Geneve: Leiden (E. J. Brill), 199-421.

Borsetti, A. M., 1959. Tre Nuovi Foraminiferi Planctonici dell'Oligocene Piacentino., G. Geol., 27:205-212.

Kennett, J. P., and Srinivasan, M. S., 1983. Neogene planktonic foraminifera: New York (Hutchinson Ross).

LeRoy, L. W., 1944. Miocene foraminifera from Sumatra and Java, Netherlands East Indies. Q. Colo. Sch. Mines, 39(3):1-113.

Date of initial receipt: 16 November 1989

Date of acceptance: 12 January 1990

Ms 115B-143

\section{SPECIES LIST}

Most of the references cited in the species list may be found in Kennett and Srinivasan (1983). Those not given by these authors are listed in this paper.

\section{Beella digitata (Brady)}

(Plate 1, Fig. 1)

Globigerina digitata Brady, 1879, p. 599, Pl. 80, Figs. 6-10.

Candeina nitida (d'Orbigny)

(Plate 1, Fig. 2)

Candeina nitida d'Orbigny, 1839, p. 107, Pl. 2, Figs. 27-28.

Cassigerinella chipolensis (Cushman and Ponton)

Cassigerinella chipolensis Cushman and Ponton, 1932, p. 98 , Pl. 15, Fig. 2.

Catapsydrax dissimilis (Cushman and Bermudez) (Plate 1, Figs. 3 and 4)

Globigerina dissimilis Cushman and Bermudez, 1937, p. 25, Pl. 3, Figs. 4-6.

Catapsydrax stainforthi (Bolli, Loeblich, and Tappan)

(Plate 1, Figs. 5 and 10)

Catapsydrax stainforthi Bolli, Loeblich, and Tappan, 1957, p. 37, Pl. 7, Fig. 11.

Clavatorella bermudezi Bolli

(Plate 6, Figs. 1-3)

Hastigerinella bermudezi Bolli, 1957, p. 112, Pl. 25, Fig. 1. 
Table 1. Holes from Leg 115 analyzed for Neogene planktonic foraminifers.

\begin{tabular}{|c|c|c|c|c|c|c|}
\hline \multirow[b]{2}{*}{ Geographical area } & \multirow[b]{2}{*}{ Hole } & \multirow[b]{2}{*}{ Latitude } & \multirow[b]{2}{*}{ Longitude } & \multirow[b]{2}{*}{$\begin{array}{l}\text { Water depth } \\
\text { (m) }\end{array}$} & \multicolumn{2}{|c|}{ Neogene sequences } \\
\hline & & & & & Cores & $\begin{array}{l}\text { Approximate } \\
\text { thickness (m) }\end{array}$ \\
\hline \multirow{2}{*}{ Southern Mascarene Plateau } & $705 \mathrm{~A}$ & $13^{\circ} 10.02^{\prime} \mathrm{S}$ & $61^{\circ} 23.02^{\prime} \mathrm{E}$ & 2320.3 & $1 \mathrm{H}-3 \mathrm{H}$ & \multirow{2}{*}{$\begin{array}{l}27 \\
3.3\end{array}$} \\
\hline & $706 \mathrm{~A}$ & $13^{\circ} 06.85^{\prime} \mathrm{S}$ & $61^{\circ} 22.26^{\prime} \mathrm{E}$ & 2504.3 & $1 \mathrm{H}-2 \mathrm{H}$ & \\
\hline \multirow{5}{*}{$\begin{array}{l}\text { Northern Mascarene } \\
\text { Plateau-Madingley Rise }\end{array}$} & $707 \mathrm{~A}$ & $07^{\circ} 32.72^{\prime} \mathrm{S}$ & $59^{\circ} 01.01^{\prime} \mathrm{E}$ & 1552.3 & $1 \mathrm{H}-15 \mathrm{H}$ & 142 \\
\hline & $708 \mathrm{~A}$ & $05^{\circ} 27.35^{\prime} \mathrm{S}$ & $59^{\circ} 56.63^{\prime} \mathrm{E}$ & 4109.3 & $1 \mathrm{H}-8 \mathrm{H}, 9 \mathrm{X}-20 \mathrm{X}$ & 185 \\
\hline & $709 \mathrm{C}$ & $03^{\circ} 54.90^{\prime} \mathrm{S}$ & $60^{\circ} 33.10^{\prime} \mathrm{E}$ & 3048.8 & $1 \mathrm{H}-20 \mathrm{H}, 21 \mathrm{X}-22 \mathrm{X}$ & 203 \\
\hline & $710 \mathrm{~A}$ & $04^{\circ} 18.70^{\prime} \mathrm{S}$ & $60^{\circ} 58.80^{\prime} \mathrm{E}$ & 3824.3 & $1 \mathrm{H}-13 \mathrm{H}, 14 \mathrm{X}-16 \mathrm{X}$ & 152 \\
\hline & $711 \mathrm{~A}$ & $02^{\circ} 44.56^{\prime} \mathrm{S}$ & $61^{\circ} 09.78^{\prime} \mathrm{E}$ & 4429.8 & $1 \mathrm{H}-10 \mathrm{H}$ & 95 \\
\hline \multirow{2}{*}{ Northern Margin Chagos Bank } & $712 \mathrm{~A}$ & $04^{\circ} 12.99^{\prime} \mathrm{S}$ & $73^{\circ} 24.38^{\prime} \mathrm{E}$ & 2904.3 & $1 \mathrm{R}-6 \mathrm{R}$ & 58 \\
\hline & $713 \mathrm{~A}$ & $04^{\circ} 11.58^{\prime} \mathrm{S}$ & $73^{\circ} 23.65^{\prime} \mathrm{E}$ & 2915.3 & $1 R-4 R$ & 31 \\
\hline \multirow{3}{*}{ Maldives Ridge } & $714 \mathrm{~A}$ & $05^{\circ} 03.60^{\prime} \mathrm{N}$ & $73^{\circ} 47.20^{\prime} \mathrm{E}$ & 2038.3 & $1 \mathrm{H}-13 \mathrm{H}, 14 \mathrm{X}-22 \mathrm{X}$ & 197 \\
\hline & $715 \mathrm{~A}$ & $05^{\circ} 04.89^{\prime} \mathrm{N}$ & $73^{\circ} 49.88^{\prime} \mathrm{E}$ & 2266.3 & $1 \mathrm{R}-11 \mathrm{R}$ & 105 \\
\hline & $716 \mathrm{~B}$ & $04^{\circ} 56.00^{\prime} \mathrm{N}$ & $73^{\circ} 17.01^{\prime} \mathrm{E}$ & 544.3 & $1 \mathrm{H}-28 \mathrm{H}$ & 264 \\
\hline
\end{tabular}

$\mathrm{H}=$ advanced hydraulic piston coring, $\mathrm{X}=$ extended core barrel, and $\mathrm{R}=$ rotary coring barrel.

Dentoglobigerina altispira altispira (Cushman and Jarvis) (Plate 6, Figs. 4-6)

Globigerina altispira Cushman and Jarvis, 1936, p. 5, Pl. 1, Fig. 13.

Dentoglobigerina altispira globosa Bolli

(Plate 6, Figs. 7 and 8)

Globoquadrina altispira globosa Bolli, 1957, p. 111, Pl. 24, Figs. 9-10.

Dentoglobigerina galavisi (Bermudez)

Globigerina galavisi Bermudez, 1960, p. 1183, Pl. 4, Fig. 3.

Dentoglobigerina globularis (Bermudez)

(Plate 6, Figs. 9 and 10)

Globoquadrina altispira globularis Bermudez, 1960, p. 1311, Pl. 13,

Figs. 4-6; Blow, 1969, pp. 339-340.

Globigerina angulisuturalis Bolli

Globigerina ciperoensis angulisuturalis Bolli, 1957, p. 109, Pl. 22, Fig. 11 .

Globigerina angustiumbilicata Bolli

Globigerina ciperoensis angustiumbilicata Bolli, 1957, p. 109, Pl. 22, Figs. 12-13.

Globigerina bulloides d'Orbigny

Globigerina bulloides d'Orbigny, 1826, p. 277; Banner and Blow, 1960, Pl. 1, Figs. 1-4.

Globigerina ciperoensis Bolli

Globigerina ciperoensis Bolli, 1954, p. 1, Figs. 3-6.

Globigerina euapertura Jenkins

Globigerina euapertura Jenkins, 1960, p. 351, Pl. 1, Fig. 8.

Globigerina praebulloides Blow

Globigerina praebulloides Blow, 1959, p. 180, Pl. 8, Fig. 47; Pl. 9, Fig. 48.

Globigerina quinqueloba Natland

Globigerina quinqueloba Natland, 1938, p. 149, Pl. 6, Fig. 7.
Globigerinatella insueta (Cushman and Stainforth)

Globigerinatella insueta Cushman and Stainforth, 1945, p. 69, Pl. 13, Figs. 7-9.

Globigerinella adamsi (Banner and Blow)

(Plate 1, Figs. 11 and 12)

Hastigerina (Bolliella) adamsi Banner and Blow, 1959, Fig. 4.

Globigerinella calida (Parker)

(Plate 1, Figs. 13-15)

Globigerina calida Parker, 1962, p. 221, PI. 1, Figs. 9-13 and 15.

Globigerinella praesiphonifera (Blow)

Hastigerina $(H$.$) siphonifera praesiphonifera Blow, 1969, p. 408, Pl. 54,$ Figs. 7-9.

Globigerinella siphonifera (d'Orbigny)

(Plate 1, Figs. 16 and 17)

Globigerina siphonifera d'Orbigny, 1839, PI. 4, Figs. 15-18.

Globigerina aequilateralis Brady, 1884, Pl. 80, Figs. 18-21.

Hastigerina $(H$.) siphonifera (d'Orbigny), Banner and Blow, 1960, Figs. 2 (lectotype), 3.

Globigerinita glutinata (Egger)

(Plate 1, Figs. 8 and 9)

Globigerina glutinata Egger, 1893, p. 371, Pl. 13, Figs. 19-21.

Globigerinoides altiaperturus Bolli

(Plate 1, Figs. 18-20)

Globigerinoides triloba altiapertura Bolli, 1957, p. 113, Pl. 25, Fig. 7.

Globigerinoides bisphericus Todd

Globigerinoides bispherica Todd, 1954, in Todd et al., 1954, p. 681, Pl. 1, Figs. 1 and 4.

Globigerinoides conglobatus (Brady)

(Plate 1, Figs. 21 and 22)

Globigerina conglobata Brady, 1879, p. 28b; 1884, Pl. 80, Figs. 1-5.

Globigerinoides diminutus Bolli

(Plate 1, Figs. 23-25)

Globigerinoides diminuta Bolli, 1957, p. 114, Pl. 25, Fig. 11. 
Table 2. Percentages of the sand-size fraction $(>63 \mu \mathrm{m})$ in Neogene sediments of Holes 705A, 706A, 707A, 708A, 709C, 710A, 711A, 712A, $713 \mathrm{~A}, 714 \mathrm{~A}, 715 \mathrm{~A}$, and $716 \mathrm{~B}$.

\begin{tabular}{|c|c|c|c|c|c|}
\hline \multirow{3}{*}{\multicolumn{3}{|c|}{$\begin{array}{l}\text { ments of Holes 705A, 706A, 707A, } \\
708 \mathrm{~A}, 709 \mathrm{C}, 710 \mathrm{~A}, 711 \mathrm{~A}, 712 \mathrm{~A} \text {, } \\
713 \mathrm{~A}, 714 \mathrm{~A}, 715 \mathrm{~A} \text {, and 716B. }\end{array}$}} & \\
\hline & & & $\begin{array}{l}\text { Core, section, } \\
\text { interval }(\mathrm{cm})\end{array}$ & $\begin{array}{l}\text { Depth } \\
\text { (mbsf) }\end{array}$ & $\begin{array}{c}>63 \mu \mathrm{m} \\
(\%)\end{array}$ \\
\hline & & & 115-708A- (Cont.) & & \\
\hline \multirow{2}{*}{$\begin{array}{l}\text { Core, section, } \\
\text { interval }(\mathrm{cm})\end{array}$} & Depth & $>63 \mu \mathrm{m}$ & $6 \mathrm{H}-3,105-110$ & 50.42 & 5.3 \\
\hline & (mbsf) & $(\%)$ & $6 \mathrm{H}-5,105-110$ & 53.15 & 5.2 \\
\hline \multirow{2}{*}{$115-707 \mathrm{~A}$ - } & & & $\begin{array}{l}6 \mathrm{H}-7,105-110 \\
7 \mathrm{H}-2,105-110\end{array}$ & $\begin{array}{l}55.65 \\
59.56\end{array}$ & $\begin{array}{l}4.2 \\
5.2\end{array}$ \\
\hline & & & $7 \mathrm{H}-3,105-110$ & 60.85 & 7.8 \\
\hline $2 \mathrm{H}-5,107-112$ & 13.67 & 76.2 & $7 \mathrm{H}-4,105-110$ & 61.54 & 6.1 \\
\hline $2 \mathrm{H}-6,107-112$ & 15.17 & 74.2 & $7 \mathrm{H}-5,105-110$ & 64.15 & 3.0 \\
\hline $3 \mathrm{H}-1,107-112$ & 17.27 & 82.4 & $7 \mathrm{H}-6,105-110$ & 65.30 & 4.0 \\
\hline $3 \mathrm{H}-2,107-112$ & 18.77 & 71.8 & $7 \mathrm{H}-7,105-110$ & 66.03 & 3.4 \\
\hline $3 \mathrm{H}-3,107-112$ & 20.27 & 68.2 & $\mathrm{sH}-1,105-110$ & 67.45 & 3.5 \\
\hline $3 \mathrm{H}-4,107-112$ & 21.77 & 67.9 & $8 \mathrm{H}-2,105-110$ & 68.95 & 4.0 \\
\hline $4 \mathrm{H}-1,107-112$ & 26.87 & 77.7 & $8 \mathrm{H}-6,105-110$ & 74.95 & 2.8 \\
\hline $4 \mathrm{H}-2,107-112$ & 28.37 & 67.0 & $9 \mathrm{H}-1,65-70$ & 76.65 & 2.8 \\
\hline $4 \mathrm{H}-3,107-112$ & 29.87 & 61.1 & $9 \mathrm{H}-\mathrm{CC}, 22-27$ & 79.31 & 1.9 \\
\hline $4 \mathrm{H}-4,107-112$ & 31.37 & 56.4 & $10 \mathrm{H}-1,106-111$ & 83.16 & 1.7 \\
\hline $5 \mathrm{H}-1,107-112$ & 36.57 & 65.7 & $10 \mathrm{H}-2,106-111$ & 84.66 & 1.5 \\
\hline SH-2, 107-112 & 38.07 & 62.3 & $12 \mathrm{H}-2,105-110$ & 103.95 & 3.0 \\
\hline $5 \mathrm{H}-3,107-112$ & 39.57 & 59.9 & $12 \mathrm{H}-3,105-110$ & 105.45 & 2.2 \\
\hline $5 \mathrm{H}-4,107-112$ & 41.07 & 52.9 & $12 \mathrm{H}-4,105-110$ & 106.95 & 2.3 \\
\hline $5 \mathrm{H}-5,107-112$ & 42.57 & 57.4 & $13 \mathrm{H}-1,12-16$ & 111.12 & 3.0 \\
\hline $5 \mathrm{H}-6,41-45$ & 43.41 & 55.5 & $13 \mathrm{H}-2,100-104$ & 112.46 & 2.6 \\
\hline $6 \mathrm{H}-1,107-112$ & 46.17 & 62.0 & $13 \mathrm{H}-3,46-50$ & 113.42 & 1.3 \\
\hline $6 \mathrm{H}-2,107-112$ & 47.67 & 57.3 & $13 \mathrm{H}-4,9-13$ & 114.55 & 1.8 \\
\hline $6 \mathrm{H}-3,107-112$ & 49.17 & 58.1 & $13 \mathrm{H}-5,83-86$ & 116.79 & 2.5 \\
\hline $6 \mathrm{H}-4,107-112$ & 50.67 & 58.1 & $14 \mathrm{H}-1,105-110$ & 121.65 & 1.1 \\
\hline $6 \mathrm{H}-5,107-112$ & 52.17 & 48.4 & $14 \mathrm{H}-2,105-110$ & 123.15 & 1.5 \\
\hline $6 \mathrm{H}-6,107-112$ & 53.67 & 52.4 & $14 \mathrm{H}-3,105-110$ & 124.65 & 3.3 \\
\hline $7 \mathrm{H}-1,107-112$ & 55.77 & 61.5 & $14 \mathrm{H}-4,105-110$ & 126.15 & 1.2 \\
\hline $7 \mathrm{H}-2,107-112$ & 57.27 & 49.7 & $14 \mathrm{H}-5,105-110$ & 127.65 & 1.2 \\
\hline $7 \mathrm{H}-3,107-112$ & 58.77 & 48.8 & $14 \mathrm{H}-6,135-140$ & 129.45 & 1.0 \\
\hline $7 \mathrm{H}-4,107-112$ & 60.27 & 39.1 & $15 \mathrm{H}-5,105-110$ & 137.35 & 0.5 \\
\hline $7 \mathrm{H}-5,107-112$ & 61.77 & 57.0 & $16 \mathrm{H}-5,108-112$ & 147.08 & 0.4 \\
\hline $7 \mathrm{H}-6,107-112$ & 63.27 & 44.7 & $17 \mathrm{H}-2,105-110$ & 152.15 & 0.8 \\
\hline $8 \mathrm{H}-1,107-112$ & 65.37 & 45.6 & $17 \mathrm{H}-3,105-110$ & 153.65 & 0.5 \\
\hline $8 \mathrm{H}-2,107-112$ & 66.87 & 49.5 & $17 \mathrm{H}-4,105-110$ & 155.15 & 0.7 \\
\hline $8 \mathrm{H}-3,107-112$ & 68.37 & 44.6 & $18 \mathrm{H}-3,105-110$ & 163.35 & 0.8 \\
\hline $8 \mathrm{H}-4,107-112$ & 69.87 & 49.0 & $19 \mathrm{H}-1,105-110$ & 169.65 & 5.0 \\
\hline $6 \mathrm{H}-5,107-112$ & 71.37 & 52.2 & $20 \mathrm{H}-3,7-11$ & 181.27 & 1.3 \\
\hline $8 \mathrm{H}-6,107-112$ & 72.87 & 44.3 & \multirow{2}{*}{\multicolumn{3}{|c|}{$115-709 \mathrm{C}-$}} \\
\hline $9 \mathrm{H}-1,107-112$ & 75.07 & 39.4 & & & \\
\hline $9 \mathrm{H}-2,107-112$ & 76.57 & 40.9 & & & \\
\hline $9 \mathrm{H}-3,107-112$ & 78.07 & 39.5 & $4 \mathrm{H}-1,60-65$ & 25.70 & 12.4 \\
\hline $9 \mathrm{H}-4,107-112$ & 79.57 & 45.0 & $4 \mathrm{H}-2,60-65$ & 27.20 & 12.2 \\
\hline $9 \mathrm{H}-5,107-112$ & 81.07 & 41.2 & $4 \mathrm{H}-4,60-65$ & 3020 & 6.9 \\
\hline $9 \mathrm{H}-6,107-112$ & 82.57 & 43.4 & $4 \mathrm{H}-5,60-65$ & 31.70 & 32.0 \\
\hline $10 \mathrm{H}-1,107-112$ & 84.67 & 52.8 & $4 \mathrm{H}-6,60-66$ & 33.20 & 11.2 \\
\hline $10 \mathrm{H}-2,107-112$ & 86.17 & 51.1 & $5 \mathrm{H}-1,60-65$ & 35.30 & 13.8 \\
\hline $10 \mathrm{H}-3,107-112$ & 87.67 & 47.0 & $5 \mathrm{H}-2,60-65$ & 36.80 & 21.5 \\
\hline $10 \mathrm{H}-4,107-112$ & 89.17 & 50.9 & $5 \mathrm{H}-3,60-65$ & 38.30 & 13.3 \\
\hline $10 \mathrm{H}-5,107-112$ & 90.67 & 54.5 & $5 \mathrm{H}-4,60-65$ & 39.80 & 12.6 \\
\hline $10 \mathrm{H}-6,80-85$ & 91.90 & 45.7 & $5 \mathrm{H}-5,60-65$ & 41.30 & 11.5 \\
\hline $11 \mathrm{H}-1,53-58$ & 93.73 & 50.3 & $5 \mathrm{H}-6,60-65$ & 42.80 & 11.2 \\
\hline $12 \mathrm{H}-1,107-112$ & 103.87 & 53.7 & $6 \mathrm{H}-1,60-65$ & 44.90 & 9.4 \\
\hline $12 \mathrm{H}-2,107-112$ & 105.37 & 59.0 & $6 \mathrm{H}-2,60-65$ & 46.40 & 9.5 \\
\hline $12 \mathrm{H}-3,107-112$ & 106.87 & 52.7 & $6 \mathrm{H}-3,60-65$ & 47.90 & 13.3 \\
\hline $12 \mathrm{H}-4,107-112$ & 108.37 & 43.1 & $6 \mathrm{H}-4,60-65$ & 49.40 & 8.0 \\
\hline $12 \mathrm{H}-5,107-112$ & 109.87 & 56.0 & $6 \mathrm{H}-5,60-65$ & 50.90 & 5.3 \\
\hline $12 \mathrm{H}-6,105-110$ & 111.35 & 59.6 & $6 \mathrm{H}-6,60-65$ & 52.40 & 11.8 \\
\hline $13 \mathrm{H}-1,107-112$ & 113.47 & 54.7 & $6 \mathrm{H}-7,60-65$ & 53.90 & 8.6 \\
\hline $13 \mathrm{H}-2,107-112$ & 114.97 & 54.3 & $7 \mathrm{H}-1,60-65$ & 54.50 & 16.4 \\
\hline $13 \mathrm{H}-3,107-112$ & 116.47 & 54.6 & $7 \mathrm{H}-2,60-65$ & 56.00 & 12.2 \\
\hline $13 \mathrm{H}-4,107-112$ & 11797 & 53.8 & $7 \mathrm{H}-3,60-65$ & 57.50 & 14.0 \\
\hline $13 \mathrm{H}-5,107-112$ & 119.47 & 42.9 & $7 \mathrm{H}-4,60-65$ & 59.00 & 8.2 \\
\hline $14 \mathrm{H}-1,107-112$ & 123.07 & 42.3 & $7 \mathrm{H}-5,60-65$ & 60.50 & 6.4 \\
\hline $14 \mathrm{H}-2,107-112$ & 124.57 & 44.5 & $7 \mathrm{H}-6,60-65$ & 62.00 & 4.7 \\
\hline $14 \mathrm{H}-3,107-112$ & 126.07 & 58.1 & $8 \mathrm{H}-2,60-65$ & 65.70 & 4.7 \\
\hline $14 \mathrm{H}-4,107-112$ & 127.57 & 64.4 & $8 \mathrm{H}-3,60-65$ & 67.20 & 5.9 \\
\hline $15 \mathrm{H}-1,107-112$ & 132.67 & 61.9 & $8 \mathrm{H}-4,60-65$ & 68.70 & 4.5 \\
\hline $15 \mathrm{H}-2,107-112$ & 134.17 & 43.6 & $8 \mathrm{H}-5,60-65$ & 70.20 & 5.9 \\
\hline $15 \mathrm{H}-3,107-112$ & 135.67 & 56.4 & $8 \mathrm{H}-6,60-65$ & 71.70 & 7.5 \\
\hline $15 \mathrm{H}-4,107-112$ & 137.17 & 47.7 & $8 \mathrm{H}-7,60-62$ & 73.20 & 11.8 \\
\hline \multirow[t]{2}{*}{$15 \mathrm{H}-5,107-112$} & 138.67 & 44.6 & $9 \mathrm{H}-2,60-65$ & 75.30 & 5.7 \\
\hline & & & $9 \mathrm{H}-3,60-65$ & 76.80 & 4.3 \\
\hline \multirow{2}{*}{ 115-708A- } & & & $9 \mathrm{H}-4,60-65$ & 78.30 & 5.8 \\
\hline & & & $9 \mathrm{H}-5,60-65$ & 79.80 & 4.3 \\
\hline $6 \mathrm{H}-2,105-110$ & 49.75 & 6.0 & $9 \mathrm{H}-6,60-65$ & 81.30 & 5.1 \\
\hline
\end{tabular}

Table 2 (continued).
Table 2 (continued).

\begin{tabular}{|c|c|c|}
\hline $\begin{array}{l}\text { Core, section, } \\
\text { interval }(\mathrm{cm})\end{array}$ & $\begin{array}{l}\text { Depth } \\
\text { (mbsf) }\end{array}$ & $\begin{array}{c}>63 \mu \mathrm{m} \\
(\%)\end{array}$ \\
\hline \multicolumn{3}{|l|}{ 115-709C-(Cont.) } \\
\hline $10 \mathrm{H}-1,60-65$ & 83.50 & 4.8 \\
\hline $10 \mathrm{H}-2,60-65$ & 85.00 & 5.0 \\
\hline $10 \mathrm{H}-3,60-65$ & 86.50 & 4.3 \\
\hline $10 \mathrm{H}-4,60-65$ & 88.00 & 3.2 \\
\hline $10 \mathrm{H}-5,60-65$ & 89.50 & 2.6 \\
\hline $10 \mathrm{H}-6,60-65$ & 91.00 & 12.5 \\
\hline $11 \mathrm{H}-1,60-65$ & 93.10 & 4.1 \\
\hline $11 \mathrm{H}-2,60-65$ & 94.60 & 5.6 \\
\hline $11 \mathrm{H}-3,60-65$ & 96.10 & 7.1 \\
\hline $11 \mathrm{H}-4,60-65$ & 97.60 & 6.8 \\
\hline $11 \mathrm{H}-5,60-65$ & 99.10 & 17.6 \\
\hline $12 \mathrm{H}-1,60-62$ & 102.80 & 13.2 \\
\hline $12 \mathrm{H}-1,120-125$ & 103.40 & 12.4 \\
\hline $12 \mathrm{H}-2,60-62$ & 104.30 & 12.5 \\
\hline $12 \mathrm{H}-2,120-125$ & 104.90 & 10.4 \\
\hline $12 \mathrm{H}-3,60-62$ & 105.80 & 10.7 \\
\hline $12 \mathrm{H}-3,120-125$ & 106.40 & 6.8 \\
\hline $12 \mathrm{H}-4,60-62$ & 107.30 & 19.9 \\
\hline $12 \mathrm{H}-4,120-125$ & 107.90 & 15.6 \\
\hline $12 \mathrm{H}-5,120-125$ & 109.40 & 20.5 \\
\hline $12 \mathrm{H}-6,60-62$ & 110.30 & 17.0 \\
\hline $12 \mathrm{H}-6,120-125$ & 110.90 & 17.3 \\
\hline $13 \mathrm{H}-1,60-62$ & 112.40 & 15.1 \\
\hline $13 \mathrm{H}-1,120-125$ & 113.00 & 6.1 \\
\hline $13 \mathrm{H}-2,60-62$ & 113.90 & 11.6 \\
\hline $13 \mathrm{H}-2,120-125$ & 114.50 & 8.2 \\
\hline $13 \mathrm{H}-3,60-62$ & 115.40 & 4.3 \\
\hline $13 \mathrm{H}-3,120-125$ & 116.00 & 4.1 \\
\hline $13 \mathrm{H}-4,60-62$ & 116.90 & 3.6 \\
\hline $13 \mathrm{H}-6,120-125$ & 120.50 & 9.5 \\
\hline $14 \mathrm{H}-1,60-62$ & 122.00 & 4.0 \\
\hline $14 \mathrm{H}-1,120-125$ & 122.60 & 6.2 \\
\hline $14 \mathrm{H}-2,60-62$ & 123.50 & 8.1 \\
\hline $14 \mathrm{H}-2,120-125$ & 124.10 & 10.9 \\
\hline $14 \mathrm{H}-3,60-62$ & 125.00 & 17.0 \\
\hline $14 \mathrm{H}-3,120-125$ & 125.60 & 10.5 \\
\hline $14 \mathrm{H}-4,60-62$ & 126.50 & 13.4 \\
\hline $14 \mathrm{H}-4,120-125$ & 127.10 & 6.6 \\
\hline $14 \mathrm{H}-5,60-62$ & 128.00 & 10.1 \\
\hline $14 \mathrm{H}-5,120-125$ & 128.60 & 7.7 \\
\hline $14 \mathrm{H}-6,60-62$ & 129.50 & 16.0 \\
\hline $14 \mathrm{H}-6,120-125$ & 130.10 & 5.7 \\
\hline $15 \mathrm{H}-1,60-65$ & 131.70 & 9.3 \\
\hline $15 \mathrm{H}-1,120-125$ & 132.30 & 13.0 \\
\hline $15 \mathrm{H}-2,60-65$ & 133.20 & 6.4 \\
\hline $15 \mathrm{H}-2,120-125$ & 133.80 & 8.6 \\
\hline $15 \mathrm{H}-3,60-65$ & 134.70 & 7.3 \\
\hline $15 \mathrm{H}-3,120-125$ & 135.30 & 2.9 \\
\hline $15 \mathrm{H}-4,60-65$ & 136.20 & 4.1 \\
\hline $15 \mathrm{H}-4,120-125$ & 136.80 & 8.6 \\
\hline $15 \mathrm{H}-5,60-65$ & 137.70 & 6.7 \\
\hline $15 \mathrm{H}-5,120-125$ & 138.30 & 5.8 \\
\hline $15 \mathrm{H}-6,60-65$ & 139.20 & 6.5 \\
\hline $15 \mathrm{H}-6,120-125$ & 139.80 & 3.7 \\
\hline $15 \mathrm{H}-7,25-30$ & 140.35 & 4.3 \\
\hline $16 \mathrm{H}-1,60-65$ & 141.40 & 11.0 \\
\hline $16 \mathrm{H}-1,120-125$ & 142.00 & 8.7 \\
\hline $16 \mathrm{H}-2,60-65$ & 142.90 & 7.8 \\
\hline $16 \mathrm{H}-2,120-125$ & 143.50 & 9.7 \\
\hline $16 \mathrm{H}-3,60-65$ & 144.40 & 2.6 \\
\hline $16 \mathrm{H}-3,120-125$ & 145.00 & 8.1 \\
\hline $16 \mathrm{H}-4,60-65$ & 145.90 & 8.5 \\
\hline $16 \mathrm{H}-4,120-125$ & 146.50 & 11.5 \\
\hline $16 \mathrm{H}-5,60-65$ & 147.40 & 6.8 \\
\hline $16 \mathrm{H}-5,120-125$ & 148.00 & 9.7 \\
\hline $16 \mathrm{H}-6,60-65$ & 148.90 & 12.7 \\
\hline $16 \mathrm{H}-6,120-125$ & 149.50 & 17.9 \\
\hline $16 \mathrm{H}-7,60-65$ & 150.40 & 10.0 \\
\hline $17 \mathrm{H}-1,60-65$ & 151.10 & 9.1 \\
\hline $17 \mathrm{H}-1,120-125$ & 151.70 & 9.1 \\
\hline $17 \mathrm{H}-2,60-65$ & 152.60 & 4.8 \\
\hline $17 \mathrm{H}-2,120-125$ & 153.20 & 5.6 \\
\hline $17 \mathrm{H}-3,60-65$ & 154.10 & 4.9 \\
\hline $17 \mathrm{H}-3,120-125$ & 154.70 & 6.0 \\
\hline $17 \mathrm{H}-4,60-65$ & 155.60 & 6.5 \\
\hline $17 \mathrm{H}-4,120-125$ & 156.20 & 5.7 \\
\hline
\end{tabular}


Table 2 (continued).

\begin{tabular}{|c|c|c|}
\hline $\begin{array}{l}\text { Core, section, } \\
\text { interval }(\mathrm{cm})\end{array}$ & $\begin{array}{l}\text { Depth } \\
\text { (mbsf) }\end{array}$ & $\begin{array}{c}>63 \mu \mathrm{m} \\
(\%)\end{array}$ \\
\hline \multicolumn{3}{|l|}{ 115-709C- (Cont.) } \\
\hline $17 \mathrm{H}-5,60-65$ & 157.10 & 20.5 \\
\hline $17 \mathrm{H}-5,120-125$ & 157.70 & 24.5 \\
\hline $17 \mathrm{H}-6,60-65$ & 158.60 & 8.5 \\
\hline $17 \mathrm{H}-6,120-125$ & 159.20 & 8.5 \\
\hline $18 \mathrm{H}-1,60-65$ & 160.80 & 9.9 \\
\hline $18 \mathrm{H}-1,120-125$ & 161.40 & 10.7 \\
\hline $18 \mathrm{H}-2,60-65$ & 162.30 & 5.5 \\
\hline $18 \mathrm{H}-2,120-125$ & 162.90 & 10.4 \\
\hline $18 \mathrm{H}-3,60-65$ & 163.80 & 11.2 \\
\hline $18 \mathrm{H}-3,120-125$ & 164.40 & 10.6 \\
\hline $18 \mathrm{H}-4,60-65$ & 165.30 & 33.2 \\
\hline $18 \mathrm{H}-4,120-125$ & 165.90 & 18.1 \\
\hline $18 \mathrm{H}-6,120-125$ & 168.90 & 7.6 \\
\hline $18 \mathrm{H}-7,60-65$ & 169.80 & 8.4 \\
\hline $19 \mathrm{H}-1,60-65$ & 170.50 & 4.8 \\
\hline $19 \mathrm{H}-1,120-125$ & 171.10 & 7.4 \\
\hline $19 \mathrm{H}-2,60-65$ & 172.00 & 4.5 \\
\hline $19 \mathrm{H}-2,120-125$ & 172.60 & 8.9 \\
\hline $19 \mathrm{H}-3,60-65$ & 173.50 & 6.8 \\
\hline $19 \mathrm{H}-3,120-125$ & 174.10 & 9.4 \\
\hline $19 \mathrm{H}-4,60-65$ & 175.00 & 5.2 \\
\hline $19 \mathrm{H}-4,120-125$ & 175.60 & 10 \\
\hline $19 \mathrm{H}-5,60-65$ & 176.50 & 7.8 \\
\hline $19 \mathrm{H}-5,120-125$ & 177.10 & 6.2 \\
\hline $19 \mathrm{H}-6,60-65$ & 178.00 & 9.6 \\
\hline $19 \mathrm{H}-6,120-125$ & 178.60 & 10.2 \\
\hline $19 \mathrm{H}-7,60-65$ & 179.50 & 6.4 \\
\hline $20 \mathrm{H}-1,60-65$ & 180.10 & 10.1 \\
\hline $20 \mathrm{H}-1,120-125$ & 180.70 & 11.3 \\
\hline $20 \mathrm{H}-2,60-65$ & 181.60 & 5.3 \\
\hline $20 \mathrm{H}-2,120-125$ & 182.20 & 3.4 \\
\hline $20 \mathrm{H}-3,60-65$ & 183.10 & 4.8 \\
\hline $20 \mathrm{H}-3,120-125$ & 183.70 & 9.5 \\
\hline $20 \mathrm{H}-4,60-65$ & 184.60 & 3.6 \\
\hline $20 \mathrm{H}-4,120-125$ & 185.20 & 8.1 \\
\hline $20 \mathrm{H}-5,60-65$ & 186.10 & 8.2 \\
\hline $20 \mathrm{H}-5,120-125$ & 186.70 & 6.9 \\
\hline $20 \mathrm{H}-6,60-65$ & 187.60 & 10.7 \\
\hline $20 \mathrm{H}-6,120-125$ & 188.20 & 12.8 \\
\hline $20 \mathrm{H}-7,60-65$ & 189.10 & 7.5 \\
\hline $21 X-1,60-65$ & 189.70 & 9.1 \\
\hline $21 \mathrm{X}-1,120-125$ & 190.30 & 7.9 \\
\hline $21 X-2,60-65$ & 191.20 & 8.5 \\
\hline $21 X-2,120-125$ & 191.80 & 6.9 \\
\hline $21 X-3,60-65$ & 192.70 & 10.8 \\
\hline $21 X-3,120-125$ & 193.30 & 8.3 \\
\hline $21 X-4,60-65$ & 194.20 & 9.7 \\
\hline $21 X-4,120-125$ & 194.80 & 10.6 \\
\hline $21 X-5,60-65$ & 195.70 & 9.0 \\
\hline $21 X-5,120-125$ & 196.30 & 13.1 \\
\hline $21 X-6,60-65$ & 197.20 & 10.1 \\
\hline $21 X-6,120-125$ & 197.80 & 10.5 \\
\hline $22 X-1,60-65$ & 199.30 & 6.6 \\
\hline $22 X-1,120-125$ & 199.90 & 6.2 \\
\hline $22 X-2,60-65$ & 200.80 & 8.3 \\
\hline $22 X-2,120-125$ & 201.40 & 8.5 \\
\hline $22 X-3,60-65$ & 202.30 & 5.5 \\
\hline $22 X-3,120-125$ & 202.90 & 9.3 \\
\hline $22 \times-4,60-65$ & 203.80 & 7.4 \\
\hline $22 X-4,120-125$ & 204.40 & 10.7 \\
\hline $22 X-5,60-65$ & 205.30 & 6.8 \\
\hline $22 X-5,120-125$ & 205.90 & 8.8 \\
\hline
\end{tabular}

\section{5-710A-}

$\begin{array}{lrr}1 \mathrm{H}-1,105-110 & 1.05 & 12.3 \\ 1 \mathrm{H}-2,90-95 & 2.40 & 11.9 \\ 1 \mathrm{H}-3,105-110 & 4.05 & 10.2 \\ 1 \mathrm{H}-4,105-110 & 5.55 & 24.5 \\ 1 \mathrm{H}-5,105-110 & 7.05 & 10.3 \\ 1 \mathrm{H}-6,105-110 & 8.55 & 12.4 \\ 2 \mathrm{H}-1,105-110 & 10.55 & 33.6 \\ 2 \mathrm{H}-2,105-110 & 12.05 & 15.1 \\ 2 \mathrm{H}-3,105-110 & 13.55 & 16.0 \\ 2 \mathrm{H}-4,105-110 & 15.05 & 20.4\end{array}$

Table 2 (continued).

\begin{tabular}{|c|c|c|}
\hline $\begin{array}{l}\text { Core, section, } \\
\text { interval }(\mathrm{cm})\end{array}$ & $\begin{array}{l}\text { Depth } \\
\text { (mbsf) }\end{array}$ & $\begin{array}{c}>63 \mu \mathrm{m} \\
(\%)\end{array}$ \\
\hline \multicolumn{3}{|l|}{ 115-710A-(Cont.) } \\
\hline $2 \mathrm{H}-5,105-110$ & 16.55 & 15.9 \\
\hline $2 \mathrm{H}-6,105-110$ & 18.05 & 13.4 \\
\hline $4 \mathrm{H}-1,105-110$ & 29.85 & 5.3 \\
\hline $4 \mathrm{H}-3,105-110$ & 32.85 & 8.4 \\
\hline $4 \mathrm{H}-4,105-110$ & 34.35 & 5.4 \\
\hline $4 \mathrm{H}-5,105-110$ & 35.85 & 3.9 \\
\hline $6 \mathrm{H}-1,105-110$ & 48.95 & 2.9 \\
\hline $6 \mathrm{H}-4,10-15$ & 52.50 & 2.3 \\
\hline $6 \mathrm{H}-5,40-45$ & 54.30 & 2.9 \\
\hline $6 \mathrm{H}-6,40-45$ & 55.80 & 2.0 \\
\hline $7 \mathrm{H}-1,105-110$ & 58.55 & 2.7 \\
\hline $7 \mathrm{H}-2,105-110$ & 60.05 & 2.0 \\
\hline $7 \mathrm{H}-3,105-110$ & 61.55 & 2.7 \\
\hline $7 \mathrm{H}-4,105-110$ & 63.05 & 1.8 \\
\hline $7 \mathrm{H}-5,105-110$ & 64.55 & 3.4 \\
\hline $7 \mathrm{H}-6,105-110$ & 66.05 & 3.0 \\
\hline $8 \mathrm{H}-1,105-110$ & 68.15 & 4.7 \\
\hline $8 \mathrm{H}-2,105-110$ & 69.65 & 2.0 \\
\hline $8 \mathrm{H}-3,105-110$ & 71.15 & 2.0 \\
\hline $8 \mathrm{H}-4,105-110$ & 72.65 & 4.1 \\
\hline $8 \mathrm{H}-5,105-110$ & 74.15 & 4.4 \\
\hline $8 \mathrm{H}-6,105-110$ & 75.65 & 5.0 \\
\hline $9 \mathrm{H}-1,105-110$ & 77.65 & 1.6 \\
\hline $9 \mathrm{H}-2,105-110$ & 79.15 & 0.8 \\
\hline $9 \mathrm{H}-3,105-110$ & 80.65 & 0.4 \\
\hline $9 \mathrm{H}-4,105-110$ & 82.15 & 0.9 \\
\hline $9 \mathrm{H}-5,105-110$ & 83.65 & 0.6 \\
\hline $9 \mathrm{H}-6,105-110$ & 85.15 & 2.9 \\
\hline $10 \mathrm{H}-1,105-110$ & 87.25 & 0.3 \\
\hline $10 \mathrm{H}-2,105-110$ & 88.75 & 0.8 \\
\hline $10 \mathrm{H}-3,105-110$ & 90.25 & 0.2 \\
\hline $10 \mathrm{H}-4,105-110$ & 91.75 & 0.4 \\
\hline $10 \mathrm{H}-5,105-110$ & 93.25 & 1.0 \\
\hline $10 \mathrm{H}-6,105-110$ & 94.75 & 0.8 \\
\hline $11 \mathrm{H}-1,105-110$ & 96.85 & 1.8 \\
\hline $11 \mathrm{H}-2,105-110$ & 98.35 & 0.2 \\
\hline $11 \mathrm{H}-3,105-110$ & 99.85 & 0.9 \\
\hline $11 \mathrm{H}-4,105-110$ & 101.35 & 0.2 \\
\hline $11 \mathrm{H}-5,105-110$ & 102.85 & 1.4 \\
\hline $11 \mathrm{H}-6,105-110$ & 104.35 & 1.4 \\
\hline $12 \mathrm{H}-1,105-110$ & 106.55 & 0.3 \\
\hline $12 \mathrm{H}-2,105-110$ & 108.05 & 0.5 \\
\hline $12 \mathrm{H}-3,105-110$ & 109.55 & 0.7 \\
\hline $12 \mathrm{H}-4,105-110$ & 111.05 & 0.3 \\
\hline $12 \mathrm{H}-5,105-110$ & 112.55 & 0.2 \\
\hline $12 \mathrm{H}-6,105-110$ & 114.05 & 0.3 \\
\hline $13 \mathrm{H}-1,105-110$ & 116.25 & 0.1 \\
\hline $13 \mathrm{H}-2,105-110$ & 117.75 & 0.6 \\
\hline $13 \mathrm{H}-3,105-110$ & 119.25 & 0.5 \\
\hline $13 \mathrm{H}-4,105-110$ & 120.75 & 0.4 \\
\hline $13 \mathrm{H}-5,105-110$ & 122.25 & 0.3 \\
\hline $13 \mathrm{H}-6,105-110$ & 123.75 & 0.2 \\
\hline $14 \mathrm{H}-1,105-110$ & 125.95 & 0.4 \\
\hline $14 \mathrm{H}-2,105-110$ & 127.45 & 0.3 \\
\hline $14 \mathrm{H}-3,105-110$ & 128.95 & 0.4 \\
\hline $14 \mathrm{H}-5,105-110$ & 131.95 & 1.3 \\
\hline $14 \mathrm{H}-6,105-110$ & 133.45 & 5.1 \\
\hline $15 \mathrm{H}-1 \quad 105-110$ & 135.65 & 5.4 \\
\hline $15 \mathrm{H}-2,105-110$ & 137.15 & 6.1 \\
\hline $15 \mathrm{H}-3,105-110$ & 138.65 & 4.1 \\
\hline $15 \mathrm{H}-4,105-110$ & 140.15 & 4.8 \\
\hline $16 \mathrm{H}-1,105-110$ & 143.15 & 2.9 \\
\hline $16 \mathrm{H}-2,105-110$ & 144.65 & 4.8 \\
\hline $16 \mathrm{H}-3,105-110$ & 146.15 & 5.9 \\
\hline $16 \mathrm{H}-4,105-110$ & 147.65 & 4.7 \\
\hline \multicolumn{3}{|l|}{$115-711 \mathrm{~A}-$} \\
\hline $1 \mathrm{H}-1,105-110$ & 1.05 & 7.4 \\
\hline $1 \mathrm{H}-2,105-110$ & 2.55 & 11.2 \\
\hline $1 \mathrm{H}-3,105-110$ & 4.05 & 2.2 \\
\hline $1 \mathrm{H}-4,105-110$ & 5.55 & 8.4 \\
\hline $1 \mathrm{H}-5,105-110$ & 7.05 & 10.2 \\
\hline $2 \mathrm{H}-1,105-110$ & 9.15 & 7.7 \\
\hline $2 \mathrm{H}-2,105-110$ & 10.65 & 3.5 \\
\hline
\end{tabular}

Table 2 (continued).

\begin{tabular}{|c|c|c|}
\hline $\begin{array}{l}\text { Core, section, } \\
\text { interval }(\mathrm{cm})\end{array}$ & $\begin{array}{l}\text { Depth } \\
\text { (mbsf) }\end{array}$ & $\begin{array}{c}>63 \mu \mathrm{m} \\
(\%)\end{array}$ \\
\hline \multicolumn{3}{|l|}{ 115-711A- (Cont.) } \\
\hline $2 \mathrm{H}-3,105-110$ & 12.15 & 1.0 \\
\hline $3 \mathrm{H}-3,105-110$ & 21.75 & 0.1 \\
\hline $4 \mathrm{H}-3,105-110$ & 31.35 & 0.3 \\
\hline $5 \mathrm{H}-3,105-110$ & 40.95 & 0.0 \\
\hline $6 \mathrm{H}-3,105-110$ & 50.65 & 0.3 \\
\hline $7 \mathrm{H}-3,105-110$ & 60.35 & 0.1 \\
\hline $8 \mathrm{H}-3,60-62$ & 68.10 & 0.2 \\
\hline $9 \mathrm{H}-3,105-110$ & 79.65 & 4.9 \\
\hline $10 \mathrm{H}-3,105-110$ & 89.25 & 0.3 \\
\hline \multicolumn{3}{|l|}{$115-712 \mathrm{~A}-$} \\
\hline $1 \mathrm{H}-1,133-138$ & 1.33 & 31.3 \\
\hline $1 \mathrm{H}-2,133-138$ & 2.83 & 78.7 \\
\hline $1 \mathrm{H}-3,133-138$ & 4.33 & 35.3 \\
\hline $1 \mathrm{H}-4,133-138$ & 5.83 & 27.5 \\
\hline $1 \mathrm{H}-5,133-138$ & 7.33 & 24.3 \\
\hline $1 \mathrm{H}-6,133-138$ & 8.83 & 26.3 \\
\hline $2 \mathrm{H}-1,105-110$ & 10.45 & 34.5 \\
\hline $2 \mathrm{H}-2,105-110$ & 11.95 & 14.6 \\
\hline $2 \mathrm{H}-3,105-110$ & 13.45 & 11.6 \\
\hline $2 \mathrm{H}-4,105-110$ & 15.08 & 20.7 \\
\hline $2 \mathrm{H}-5,105-110$ & 16.58 & 18.5 \\
\hline $3 \mathrm{H}-1,105-110$ & 20.05 & 15.9 \\
\hline $3 \mathrm{H}-2,105-110$ & 21.55 & 16.2 \\
\hline $3 \mathrm{H}-3,105-110$ & 23.05 & 8.9 \\
\hline $3 \mathrm{H}-4,105-110$ & 24.55 & 24.2 \\
\hline $4 \mathrm{H}-2,105-110$ & 31.15 & 39.2 \\
\hline $4 \mathrm{H}-3,32-37$ & 31.92 & 49.0 \\
\hline $5 \mathrm{H}-1,105-110$ & 39.35 & 33.5 \\
\hline $5 \mathrm{H}-2,105-110$ & 40.85 & 30.2 \\
\hline $6 \mathrm{H}-1,105-110$ & 48.95 & 80.4 \\
\hline $6 \mathrm{H}-2,105-110$ & 50.45 & 62.6 \\
\hline
\end{tabular}

115-713A-

$\begin{array}{lrl}2 \mathrm{H}-2,105-110 & 4.15 & 60.4 \\ 2 \mathrm{H}-3,105-110 & 5.65 & 50.9 \\ 2 \mathrm{H}-4,105-110 & 7.15 & 42.2 \\ 2 \mathrm{H}-6,83-88 & 9.93 & 49.4 \\ 3 \mathrm{H}-1,105-110 & 12.25 & 20.6 \\ 3 \mathrm{H}-2,105-110 & 13.75 & 21.5 \\ 3 \mathrm{H}-3,105-110 & 15.25 & 22.0 \\ 3 \mathrm{H}-4,105-110 & 16.75 & 23.5 \\ 3 \mathrm{H}-5,105-110 & 18.25 & 24.9 \\ 3 \mathrm{H}-6,105-110 & 19.75 & 19.3 \\ 4 \mathrm{H}-1,105-110 & 21.85 & 15.7 \\ 4 \mathrm{H}-2,105-110 & 23-35 & 17.4 \\ 4 \mathrm{H}-3,105-110 & 24.85 & 18.2 \\ 4 \mathrm{H}-4,105-110 & 26.35 & 18.8 \\ 4 \mathrm{H}-5,105-110 & 27.85 & 18.4 \\ 4 \mathrm{H}-6,105-110 & 29.35 & 13.1\end{array}$

115-714A-

$\begin{array}{lll}3 \mathrm{H}-1,110-115 & 13.50 & 34.4 \\ 3 \mathrm{H}-2,110-115 & 15.00 & 37.9 \\ 3 \mathrm{H}-3,110-115 & 16.50 & 32.2 \\ 3 \mathrm{H}-4,110-115 & 18.00 & 36.1 \\ 3 \mathrm{H}-5,98-103 & 19.38 & 51.4 \\ 3 \mathrm{H}-5,138-143 & 19.78 & 38.1 \\ 3 \mathrm{H}-6,20-25 & 20.10 & 46.4 \\ 3 \mathrm{H}-6,110-115 & 21.00 & 45.2 \\ 4 \mathrm{H}-2,20-25 & 22.38 & 36.8 \\ 4 \mathrm{H}-2,105-110 & 23.23 & 39.2 \\ 4 \mathrm{H}-3,105-110 & 24.68 & 34.5 \\ 4 \mathrm{H}-4,20-25 & 25.33 & 33.7 \\ 4 \mathrm{H}-4,105-110 & 26.18 & 37.2 \\ 4 \mathrm{H}-5,20-25 & 26.83 & 35.4 \\ 4 \mathrm{H}-5,105-110 & 27.68 & 38.7 \\ 4 \mathrm{H}-8,20-25 & 31.33 & 37.2 \\ 5 \mathrm{H}-1,20-25 & 31.90 & 49.2 \\ 5 \mathrm{H}-1,105-110 & 32.75 & 32.0 \\ 5 \mathrm{H}-2,20-25 & 33.40 & 28.2 \\ 5 \mathrm{H}-2,105-110 & 34.25 & 31.2 \\ 5 \mathrm{H}-3,20-25 & 34.90 & 41.8\end{array}$


Table 2 (continued).

\begin{tabular}{|c|c|c|}
\hline $\begin{array}{l}\text { Core, section, } \\
\text { interval }(\mathrm{cm})\end{array}$ & $\begin{array}{l}\text { Depth } \\
\text { (mbsf) }\end{array}$ & $\begin{array}{c}>63 \mu \mathrm{m} \\
(\%)\end{array}$ \\
\hline \multicolumn{3}{|l|}{ 115-714A- (Cont.) } \\
\hline $5 \mathrm{H}-3,105-110$ & 35.75 & 25.7 \\
\hline $5 \mathrm{H}-4,20-25$ & 36.40 & 34.5 \\
\hline $5 \mathrm{H}-4,105-110$ & 37.25 & 22.7 \\
\hline $5 \mathrm{H}-5,20-25$ & 37.90 & 46.3 \\
\hline $5 \mathrm{H}-5,105-110$ & 38.75 & 24.8 \\
\hline $5 \mathrm{H}-6,20-25$ & 39.40 & 37.4 \\
\hline $5 \mathrm{H}-6,110-115$ & 40.25 & 23.1 \\
\hline $5 \mathrm{H}-7,20-25$ & 40.90 & 42.9 \\
\hline $6 \mathrm{H}-1,20-25$ & 41.60 & 26.7 \\
\hline $6 \mathrm{H}-1,110-115$ & 42.45 & 17.0 \\
\hline $6 \mathrm{H}-2,20-25$ & 43.10 & 24.7 \\
\hline $6 \mathrm{H}-2,110-115$ & 43.95 & 28.7 \\
\hline $6 \mathrm{H}-3,20-25$ & 44.60 & 24.5 \\
\hline $6 \mathrm{H}-3,110-115$ & 45.45 & 15.1 \\
\hline $6 \mathrm{H}-4,20-25$ & 46.10 & 41.6 \\
\hline $6 \mathrm{H}-4,110-115$ & 46.95 & 38.0 \\
\hline $6 \mathrm{H}-5,20-25$ & 47.60 & 24.2 \\
\hline $6 \mathrm{H}-5,110-115$ & 48.45 & 25.8 \\
\hline $6 \mathrm{H}-6,20-25$ & 49.10 & 31.6 \\
\hline $6 \mathrm{H}-6,110-115$ & 49.95 & 45.6 \\
\hline $6 \mathrm{H}-7,20-25$ & 50.60 & 39.4 \\
\hline $7 \mathrm{H}-1,20-25$ & 51.20 & 36.2 \\
\hline $7 \mathrm{H}-1,110-115$ & 52.05 & 33.3 \\
\hline $7 \mathrm{H}-2,20-25$ & 52.70 & 34.5 \\
\hline $7 \mathrm{H}-3,110-115$ & 55.05 & 35.2 \\
\hline $7 \mathrm{H} \mathrm{4,2025}$ & 55.70 & 38.2 \\
\hline $7 \mathrm{H}-4,110-115$ & 56.55 & 31.5 \\
\hline $7 \mathrm{H}-5,20-25$ & 57.20 & 32.6 \\
\hline $7 \mathrm{H}-5,110-115$ & 58.05 & 34.1 \\
\hline $7 \mathrm{H}-6,20-25$ & 58.70 & 35.0 \\
\hline $7 \mathrm{H}-6,110-115$ & 59.55 & 28.2 \\
\hline $7 \mathrm{H}-7,20-25$ & 60.20 & 31.6 \\
\hline $8 \mathrm{H}-1,20-25$ & 60.80 & 29.0 \\
\hline $8 \mathrm{H}-1,110-115$ & 61.65 & 34.5 \\
\hline $8 \mathrm{H}-2,20-25$ & 62.30 & 36.7 \\
\hline $8 \mathrm{H}-2,110-115$ & 63.15 & 41.9 \\
\hline $8 \mathrm{H}-3,20-25$ & 63.80 & 23.0 \\
\hline $8 \mathrm{H}-3,110-115$ & 64.65 & 28.8 \\
\hline $8 \mathrm{H}-4,20-25$ & 65.30 & 23.9 \\
\hline $8 \mathrm{H}-4,110-115$ & 66.15 & 27.5 \\
\hline $8 \mathrm{H}-5,20-25$ & 66.80 & 30.1 \\
\hline $8 \mathrm{H}-5,110-115$ & 67.65 & 27.4 \\
\hline $8 \mathrm{H}-6,20-25$ & 68.30 & 20.2 \\
\hline $8 \mathrm{H}-6,110-115$ & 69.15 & 28.3 \\
\hline $8 \mathrm{H}-7,20-25$ & 69.80 & 44.6 \\
\hline $9 \mathrm{H}-1,20-25$ & 70.40 & 36.5 \\
\hline $9 \mathrm{H}-1,110-115$ & 71.25 & 34.0 \\
\hline $9 \mathrm{H}-2,20-25$ & 71.90 & 29.9 \\
\hline $9 \mathrm{H}-2,110-115$ & 72.75 & 26.3 \\
\hline $9 \mathrm{H}-3,20-25$ & 73.40 & 23.7 \\
\hline $9 \mathrm{H}-3,110-115$ & 74.25 & 32.8 \\
\hline $9 \mathrm{H}-4,20-25$ & 74.90 & 21.0 \\
\hline $10 \mathrm{H}-1,20-25$ & 80.00 & 22.2 \\
\hline $10 \mathrm{H}-1,110-115$ & 80.85 & 21.7 \\
\hline $10 \mathrm{H}-2,20-25$ & 81.50 & 27.0 \\
\hline $10 \mathrm{H}-2,110-115$ & 82.35 & 31.1 \\
\hline $10 \mathrm{H}-3,20-25$ & 83.00 & 23.3 \\
\hline $10 \mathrm{H}-4,20-25$ & 84.50 & 25.1 \\
\hline $10 \mathrm{H}-4,110-115$ & 85.35 & 26.3 \\
\hline $10 \mathrm{H}-5,20-25$ & 86.00 & 30.8 \\
\hline $10 \mathrm{H}-5,110-115$ & 86.85 & 54.7 \\
\hline $10 \mathrm{H}-6,20-25$ & 87.50 & 30.2 \\
\hline $10 \mathrm{H}-6,110-115$ & 88.35 & 18.2 \\
\hline $10 \mathrm{H}-7,20-25$ & 89.00 & 30.0 \\
\hline $11 \mathrm{H}-1,20-25$ & 89.70 & 22.5 \\
\hline $11 \mathrm{H}-1,110-115$ & 90.55 & 29.2 \\
\hline $11 \mathrm{H}-2,20-25$ & 91.20 & 28.3 \\
\hline $11 \mathrm{H}-2,110-115$ & 92.05 & 30.0 \\
\hline $11 \mathrm{H}-3,20-25$ & 92.70 & 52.7 \\
\hline $11 \mathrm{H}-3,110-115$ & 93.55 & 42.7 \\
\hline $11 \mathrm{H}-4,20-25$ & 94.20 & 42.7 \\
\hline $11 \mathrm{H}-4,110-115$ & 95.05 & 37.5 \\
\hline $11 \mathrm{H}-5,20-25$ & 95.70 & 30.5 \\
\hline $11 \mathrm{H}-5,110-115$ & 96.55 & 24.7 \\
\hline $11 \mathrm{H}-6,20-25$ & 97.20 & 30.7 \\
\hline
\end{tabular}

Table 2 (continued).

\begin{tabular}{|c|c|c|}
\hline $\begin{array}{l}\text { Core, section, } \\
\text { interval }(\mathrm{cm})\end{array}$ & $\begin{array}{l}\text { Depth } \\
\text { (mbsf) }\end{array}$ & $\begin{array}{c}>63 \mu \mathrm{m} \\
(\%)\end{array}$ \\
\hline \multicolumn{3}{|l|}{ 115-714A- (Cont.) } \\
\hline $11 \mathrm{H}-6,110-115$ & 98.05 & 29.2 \\
\hline $12 \mathrm{H}-1,110-115$ & 100.15 & 23.2 \\
\hline $12 \mathrm{H}-2,20-25$ & 100.80 & 29.2 \\
\hline $12 \mathrm{H}-2,110-115$ & 101.65 & 32.2 \\
\hline $12 \mathrm{H}-3,20-25$ & 102.30 & 23.8 \\
\hline $12 \mathrm{H}-3,110-115$ & 103.15 & 19.7 \\
\hline $12 \mathrm{H}-4,20-25$ & 103.80 & 20.6 \\
\hline $12 \mathrm{H}-4,110-115$ & 104.65 & 16.7 \\
\hline $12 \mathrm{H}-5,20-25$ & 105.30 & 18.6 \\
\hline $12 \mathrm{H}-5,110-115$ & 106.15 & 33.2 \\
\hline $12 \mathrm{H}-6,20-25$ & 106.80 & 24.3 \\
\hline $12 \mathrm{H}-6,110-115$ & 107.65 & 26.7 \\
\hline $12 \mathrm{H}-7,20-25$ & 108.30 & 24.5 \\
\hline $13 \mathrm{H}-1,20-25$ & 108.90 & 16.7 \\
\hline $13 \mathrm{H}-1,110-115$ & 109.75 & 15.5 \\
\hline $13 \mathrm{H}-2,20-25$ & 110.40 & 22.3 \\
\hline $13 \mathrm{H}-2,110-115$ & 111.25 & 25.7 \\
\hline $13 \mathrm{H}-3,20-25$ & 111.90 & 19.8 \\
\hline $13 \mathrm{H}-3,110-115$ & 112.75 & 23.8 \\
\hline $13 \mathrm{H}-4,20-25$ & 113.40 & 23.0 \\
\hline $13 \mathrm{H}-4,110-115$ & 114.25 & 22.6 \\
\hline $13 \mathrm{H}-5,20-25$ & 114.90 & 22.9 \\
\hline $13 \mathrm{H}-5,110-115$ & 115.75 & 23.6 \\
\hline $13 \mathrm{H}-6,20-25$ & 116.40 & 24.6 \\
\hline $13 \mathrm{H}-6,110-115$ & 117.25 & 24.0 \\
\hline $13 \mathrm{H}-7,20-25$ & 117.90 & 29.4 \\
\hline $14 \mathrm{X}-1,20-25$ & 118.60 & 26.0 \\
\hline $14 \mathrm{X}-1,110-115$ & 119.45 & 29.5 \\
\hline $14 X-2,20-25$ & 120.10 & 33.8 \\
\hline $14 X-2,110-115$ & 120.95 & 36.0 \\
\hline $14 \mathrm{X}-3,20-25$ & 121.60 & 34.9 \\
\hline $14 \mathrm{X}-3,110-115$ & 122.45 & 31.4 \\
\hline $14 X-4,20-25$ & 123.10 & 31.9 \\
\hline $14 X-4,110-115$ & 123.95 & 31.9 \\
\hline $14 X-5,20-25$ & 124.60 & 31.7 \\
\hline $14 X-5,110-115$ & 125.45 & 34.2 \\
\hline $14 \mathrm{X}-6,20-25$ & 126.10 & 27.5 \\
\hline $14 X-6,110-115$ & 126.95 & 17.6 \\
\hline $15 \mathrm{X}-1,20-25$ & 127.00 & 26.9 \\
\hline $15 X-1,110-115$ & 127.85 & 28.5 \\
\hline $15 X-2,20-25$ & 128.50 & 26.3 \\
\hline $15 X-2,110-115$ & 129.35 & 32.0 \\
\hline $15 X-3,20-25$ & 130.00 & 21.1 \\
\hline $15 X-3,110-115$ & 130.85 & 29.8 \\
\hline $15 \times-4,20-25$ & 131.50 & 26.0 \\
\hline $15 \times-4,110-115$ & 132.35 & 28.3 \\
\hline $15 X-5,20-25$ & 133.00 & 24.8 \\
\hline $15 X-5,110-115$ & 133.85 & 32.9 \\
\hline $15 \mathrm{X}-6,20-25$ & 134.17 & 30.8 \\
\hline $16 \mathrm{X}-1,20-25$ & 136.60 & 26.4 \\
\hline $16 \mathrm{X}-1,110-115$ & 137.45 & 32.5 \\
\hline $16 \mathrm{X}-2,20-25$ & 138.10 & 32.2 \\
\hline $16 \mathrm{X}-2,110-115$ & 138.95 & 33.8 \\
\hline $16 \mathrm{X}-3,20-25$ & 139.60 & 20.9 \\
\hline $16 \mathrm{X}-3,110-115$ & 140.45 & 24.7 \\
\hline $16 X-4,20-25$ & 141.10 & 21.7 \\
\hline $16 \times-4,110-115$ & 141.95 & 26.7 \\
\hline $16 \mathrm{X}-5,20-25$ & 142.60 & 23.4 \\
\hline $16 \times-5,110-115$ & 143.45 & 31.8 \\
\hline $17 \mathrm{X}-1,20-25$ & 146.30 & 23.9 \\
\hline $17 \mathrm{X}-1,110-115$ & 147.15 & 23.5 \\
\hline $17 \times-2,20-25$ & 147.80 & 26.6 \\
\hline $17 X-2,110-115$ & 148.65 & 32.7 \\
\hline $17 X-3,20-25$ & 149.30 & 27.6 \\
\hline $17 X-3,110-115$ & 150.15 & 24.7 \\
\hline $17 \times-4,20-25$ & 150.80 & 25.4 \\
\hline $17 \times-4,110-115$ & 151.65 & 24.2 \\
\hline $17 X-5,20-25$ & 152.30 & 30.5 \\
\hline $18 \mathrm{X}-1,20-25$ & 155.90 & 29.3 \\
\hline $18 \times-1,110-115$ & 156.75 & 31.3 \\
\hline $18 X-2,20-25$ & 157.40 & 37.2 \\
\hline $18 \mathrm{X}-3,20-25$ & 158.90 & 67.6 \\
\hline $18 X-4,20-25$ & 160.40 & 34.6 \\
\hline $18 \times-4,110-115$ & 161.25 & 38.6 \\
\hline $18 X-5,20-25$ & 165.50 & 38.6 \\
\hline
\end{tabular}

Table 2 (continued).

\begin{tabular}{|c|c|c|}
\hline $\begin{array}{l}\text { Core, section, } \\
\text { interval }(\mathrm{cm})\end{array}$ & $\begin{array}{l}\text { Depth } \\
\text { (mbsf) }\end{array}$ & $\begin{array}{c}>63 \mu \mathrm{n} \\
(\%)\end{array}$ \\
\hline \multicolumn{3}{|l|}{$115-714 \mathrm{~A}-$ (Cont.) } \\
\hline $19 X-2,105-110$ & 167.85 & 30.2 \\
\hline $19 X-3,20-25$ & 168.50 & 29.1 \\
\hline $19 \times-4,20-25$ & 170.00 & 22.5 \\
\hline $19 X-4,110-115$ & 170.85 & 30.7 \\
\hline $19 X-5,20-25$ & 171.50 & 37.4 \\
\hline $19 X-6,20-25$ & 173.00 & 31.7 \\
\hline $19 X-C C, 20-25$ & 174.50 & 30.1 \\
\hline $20 \mathrm{X}-1,20-25$ & 175.20 & 33.6 \\
\hline $20 \times-3,105-110$ & 179.05 & 28.5 \\
\hline $20 \times-4,110-115$ & 180.55 & 31.6 \\
\hline $20 X-5,20-25$ & 181.20 & 31.9 \\
\hline $21 X-1,25-30$ & 184.95 & 40.0 \\
\hline $22 \mathrm{X}-1,20-25$ & 194.60 & 22.7 \\
\hline $22 X-1,110-115$ & 195.45 & 24.9 \\
\hline $22 \times-2,20-25$ & 196.10 & 11.4 \\
\hline $22 X-2,110-115$ & 196.95 & 18.3 \\
\hline $22 \times-4,20-25$ & 199.10 & 28.0 \\
\hline $22 \times-4,110-115$ & 199.95 & 20.4 \\
\hline $22 X-5,20-25$ & 200.60 & 12.4 \\
\hline $22 X-5,53-58$ & 200.93 & 23.7 \\
\hline
\end{tabular}

115-715A.

$6 \mathrm{H}-1,105-110 \quad 47.85 \quad 37.0$

$6 \mathrm{H}-2,105-110 \quad 49.35 \quad 41.1$

$6 \mathrm{H}-3,105-110 \quad 50.85 \quad 52.6$

$6 \mathrm{H}-4,105-110 \quad 52.35 \quad 45.7$

$6 \mathrm{H}-5,105-110 \quad 53.85 \quad 37.8$

$6 \mathrm{H}-6,105-110 \quad 55.35 \quad 37.9$

$7 \mathrm{H}-1,105-110 \quad 57.55 \quad 29.6$

$7 \mathrm{H}-2,105-110 \quad 59.05 \quad 32.6$

$7 \mathrm{H}-3,105-110 \quad 60.55 \quad 31.9$

$7 \mathrm{H}-4,105-110 \quad 62.05 \quad 31.1$

7H-5, $105-110 \quad 63.55 \quad 31.6$

$7 \mathrm{H}-6,105-110 \quad 65.05 \quad 41.3$

$8 \mathrm{H}-1,105-110 \quad 67.15 \quad 39.4$

$8 \mathrm{H}-2,105-110 \quad 68.65 \quad 35.4$

$8 \mathrm{H}-3,105-110 \quad 70.15 \quad 44.1$

$8 \mathrm{H}-4,105-110 \quad 71.65 \quad 40.9$

8H-5, 105-110 $\quad 73.15 \quad 42.0$

$8 \mathrm{H}-6,105-110 \quad 74.65 \quad 41.4$

$9 \mathrm{H}-1,105-110 \quad 76.75 \quad 39.7$

$9 \mathrm{H}-2,105-110 \quad 78.25 \quad 38.1$

9H-3, 105-110 $\quad 79.75 \quad 37.3$

$9 \mathrm{H}-4,105-110 \quad 81.25 \quad 38.2$

$9 \mathrm{H}-5,105-110 \quad 82.75 \quad 37.2$

9H-6, 105-110 $\quad 84.25 \quad 40.1$

$10 \mathrm{H}-2,105-110 \quad 87.85 \quad 45.2$

$\begin{array}{lll}10 \mathrm{H}-3,105-110 & 89.35 & 44.8 \\ & & \end{array}$

$\begin{array}{lll}10 \mathrm{H}-4,105-110 & 90.85 & 40.0 \\ 10 \mathrm{H}-5,105-110 & 92.35 & 32.8\end{array}$

$\begin{array}{lll}10 \mathrm{H}-6,105-110 & 93.85 & 37.8\end{array}$

$11 \mathrm{H}-1,105-110 \quad 95.95 \quad 30.0$

$11 \mathrm{H}-2,105-110 \quad 97.45 \quad 36.3$

$11 \mathrm{H}-3,105-110 \quad 98.95 \quad 36.3$

$11 \mathrm{H}-4,105-110 \quad 100.45 \quad 38.6$

$11 \mathrm{H}-5,105-110 \quad 101.95 \quad 38.6$

$11 \mathrm{H}-6,105-110 \quad 103.45 \quad 36.1$

115-716B-

$12 \mathrm{H}-1,105-108$

$12 \mathrm{H}-2,105-108$

$12 \mathrm{H}-3,105-108$

$12 \mathrm{H}-4,105-108$

$12 \mathrm{H}-5,105-108$

$12 \mathrm{H}-6,105-108$

$13 \mathrm{H}-1,105-108$

$13 \mathrm{H}-2,105-108$

$13 \mathrm{H}-3,105-108$

$13 \mathrm{H}-4,105-108$

13H-5, 105-108

$13 \mathrm{H}-6,105-108$

$14 \mathrm{H}-1,105-108$

$14 \mathrm{H}-2,105-108$

$14 \mathrm{H}-3,105-108$

101.35

102.85

$105.85 \quad 47.9$

$107.35 \quad 46.1$

$108.85 \quad 39.0$

$\begin{array}{ll}11.05 & 56.8\end{array}$

52.2

$114.05 \quad 47.3$

$\begin{array}{ll}117.05 & 56.8 \\ 117.05 & 52.5\end{array}$

$118.55 \quad 46.6$

$120.75 \quad 34.1$

$122.25 \quad 57.0$

$123.75 \quad 51.1$ 
Table 2 (continued).

\begin{tabular}{|c|c|c|}
\hline $\begin{array}{l}\text { Core, section, } \\
\text { interval }(\mathrm{cm})\end{array}$ & $\begin{array}{l}\text { Depth } \\
\text { (mbsf) }\end{array}$ & $\begin{array}{c}>\quad 63 \mu \mathrm{m} \\
(\%)\end{array}$ \\
\hline \multicolumn{3}{|l|}{ 115-716B- (Cont.) } \\
\hline $14 \mathrm{H}-4,105-108$ & 125.25 & 44.8 \\
\hline $14 \mathrm{H}-5,105-108$ & 126.75 & 45.4 \\
\hline $14 \mathrm{H}-6,105-108$ & 128.25 & 48.4 \\
\hline $15 \mathrm{H}-1,105-108$ & 130.35 & 42.6 \\
\hline $15 \mathrm{H}-2,105-108$ & 131.85 & 51.1 \\
\hline $15 \mathrm{H}-3,105-108$ & 133.35 & 46.2 \\
\hline $15 \mathrm{H}-5,105-108$ & 136.35 & 51.2 \\
\hline $15 \mathrm{H}-6,105-108$ & 137.85 & 51.0 \\
\hline $16 \mathrm{H}-1,105-108$ & 139.95 & 47.3 \\
\hline $16 \mathrm{H}-2,105-108$ & 141.45 & 50.6 \\
\hline $16 \mathrm{H}-3,105-108$ & 142.95 & 48.1 \\
\hline $16 \mathrm{H}-4,105-108$ & 144.45 & 48.7 \\
\hline $16 \mathrm{H}-5,105-108$ & 145.95 & 53.4 \\
\hline $16 \mathrm{H}-6,105-108$ & 147.45 & 52.0 \\
\hline $17 \mathrm{H}-1,105-108$ & 149.65 & 35.1 \\
\hline $17 \mathrm{H}-2,105-108$ & 151.15 & 31.6 \\
\hline $17 \mathrm{H}-3,105-108$ & 152.65 & 27.2 \\
\hline $17 \mathrm{H}-4,105-108$ & 154.15 & 34.3 \\
\hline $17 \mathrm{H}-5,105-108$ & 155.65 & 19.9 \\
\hline $17 \mathrm{H}-6,105-108$ & 157.15 & 6.9 \\
\hline $18 \mathrm{H}-1,105-108$ & 159.35 & 28.2 \\
\hline $18 \mathrm{H}-2,105-108$ & 160.85 & 50.8 \\
\hline $18 \mathrm{H}-3,105-108$ & 162.35 & 44.4 \\
\hline $18 \mathrm{H}-4,105-108$ & 163.85 & 29.9 \\
\hline $18 \mathrm{H}-5,105-108$ & 165.35 & 26.9 \\
\hline $18 \mathrm{H}-6,105-108$ & 166.85 & 24.9 \\
\hline $19 \mathrm{H}-1,105-108$ & 168.95 & 11.7 \\
\hline $19 \mathrm{H}-2,105-108$ & 170.45 & 37.0 \\
\hline $19 \mathrm{H}-3,105-108$ & 171.95 & 20.0 \\
\hline $19 \mathrm{H}-4,105-108$ & 173.45 & 8.4 \\
\hline $19 \mathrm{H}-5,105-108$ & 174.95 & 8.2 \\
\hline $19 \mathrm{H}-6,105-108$ & 176.45 & 25.8 \\
\hline $20 \mathrm{H}-1,105-108$ & 178.55 & 22.1 \\
\hline $20 \mathrm{H}-2,105-108$ & 180.05 & 31.3 \\
\hline $20 \mathrm{H}-3,105-108$ & 181.55 & 15.1 \\
\hline $20 \mathrm{H}-4,105-108$ & 183.05 & 10.1 \\
\hline $20 \mathrm{H}-5,105-108$ & 184.55 & 33.7 \\
\hline $20 \mathrm{H}-6,105-108$ & 186.05 & 25.2 \\
\hline $21 \mathrm{H}-1,105-108$ & 188.25 & 32.6 \\
\hline $21 \mathrm{H}-2,105-108$ & 189.75 & 12.7 \\
\hline $21 \mathrm{H}-3,105-108$ & 191.25 & 26.3 \\
\hline $21 \mathrm{H}-4,105-108$ & 192.75 & 38.6 \\
\hline $21 \mathrm{H}-5,105-108$ & 194.25 & 27.7 \\
\hline $21 \mathrm{H}-6,105-108$ & 195.75 & 25.9 \\
\hline $22 \mathrm{H}-1,105-108$ & 197.95 & 9.0 \\
\hline $22 \mathrm{H}-2,105-108$ & 199.45 & 12.5 \\
\hline $22 \mathrm{H}-4,105-108$ & 202.45 & 14.7 \\
\hline $22 \mathrm{H}-5,105-108$ & 203.95 & 23.1 \\
\hline $22 \mathrm{H}-6,105-108$ & 205.45 & 23.9 \\
\hline $23 \mathrm{H}-1,105-108$ & 207.55 & 23.5 \\
\hline $23 \mathrm{H}-2,105-108$ & 209.05 & 12.0 \\
\hline $23 \mathrm{H}-3,105-108$ & 210.55 & 17.0 \\
\hline $23 \mathrm{H}-4,105-108$ & 212.05 & 23.5 \\
\hline $23 \mathrm{H}-5,105-108$ & 213.55 & 22.6 \\
\hline $23 \mathrm{H}-6,105-108$ & 215.05 & 27.1 \\
\hline $24 \mathrm{H}-1,105-108$ & 217.15 & 21.8 \\
\hline $24 \mathrm{H}-2,105-108$ & 218.65 & 24.3 \\
\hline $24 \mathrm{H}-3,105-108$ & 220.15 & 11.2 \\
\hline $24 \mathrm{H}-4,105-108$ & 221.65 & 12.3 \\
\hline $24 \mathrm{H}-5,105-108$ & 223.15 & 13.5 \\
\hline $24 \mathrm{H}-6,105-108$ & 224.65 & 19.2 \\
\hline $25 \mathrm{H}-1,105-108$ & 226.85 & 16.4 \\
\hline $25 \mathrm{H}-2,105-108$ & 228.35 & 11.4 \\
\hline $25 \mathrm{H}-3,105-108$ & 229.85 & 11.6 \\
\hline $25 \mathrm{H}-4,105-108$ & 231.35 & 15.8 \\
\hline $25 \mathrm{H}-5,105-108$ & 232.85 & 9.6 \\
\hline $25 \mathrm{H}-6,105-108$ & 234.35 & 9.1 \\
\hline $26 \mathrm{H}-1,105-108$ & 236.55 & 5.3 \\
\hline $26 \mathrm{H}-2,105-108$ & 238.05 & 12.1 \\
\hline $26 \mathrm{H}-3,105-108$ & 239.55 & 7.2 \\
\hline $26 \mathrm{H}-4,105-108$ & 241.05 & 5.0 \\
\hline $26 \mathrm{H}-5,105-108$ & 242.55 & 8.2 \\
\hline $26 \mathrm{H}-6,105-108$ & 244.05 & 11.6 \\
\hline $27 \mathrm{H}-1,105-108$ & 246.15 & 6.6 \\
\hline $27 \mathrm{H}-2,105-108$ & 247.65 & 11.3 \\
\hline
\end{tabular}

Table 2 (continued).

\begin{tabular}{ccc}
\hline $\begin{array}{c}\text { Core, section, } \\
\text { interval (cm) }\end{array}$ & $\begin{array}{c}\text { Depth } \\
\text { (mbsf) }\end{array}$ & $\begin{array}{c}>63 \mu \mathrm{m} \\
(\%)\end{array}$ \\
\hline 115-716B- (Cont.) & & \\
& & \\
$27 \mathrm{H}-3,105-108$ & 249.15 & 6.8 \\
$27 \mathrm{H}-4,105-108$ & 250.65 & 9.6 \\
$27 \mathrm{H}-5,105-108$ & 252.15 & 6.3 \\
$27 \mathrm{H}-6,105-108$ & 253.65 & 4.6 \\
$28 \mathrm{H}-1,105-108$ & 255.75 & 6.4 \\
$28 \mathrm{H}-2,105-108$ & 257.25 & 5.8 \\
$28 \mathrm{H}-3,105-108$ & 258.75 & 6.4 \\
$28 \mathrm{H}-4,105-108$ & 260.25 & 2.9 \\
$28 \mathrm{H}-5,105-108$ & 261.75 & 3.5 \\
$28 \mathrm{H}-6,105-108$ & 263.25 & 6.1 \\
\hline
\end{tabular}

Globigerinoides elongatus (d'Orbigny)

(Plate 1, Figs. 26 and 27)

Globigerinoides elongatus d'Orbigny, 1826.

Globigerinoides elongatus (d'Orbigny), Parker, 1973, p. 272, Pl. 1, Fig. 9.

Globigerinoides ruber (d’Orbigny), Parker, 1967, p. 156, Pl. 22, Fig. 4.

Globigerinoides fistulosus (Schubert)

(Plate 2, Figs. 1-3)

Globigerina fistulosa Schubert, 1910, p. 323, Fig. 1.

Globigerinoides mitra Todd

(Plate 2, Figs. 4-6 and 11)

Globigerinoides mitra Todd, 1957, p. 302, Pl. 78, Figs. 3 and 6.

Globigerinoides obliquus extremus Bolli

(Plate 2, Figs. 7 and 8)

Globigerinoides obliquus extremus Bolli and Bermudez, 1965, p. 139, Pl. 1, Figs. 10-12.

Globigerinoides obliquus obliquus Bolli

(Plate 2, Figs. 9, 10, and 14)

Globigerinoides obliqua Bolli, 1957, p. 113, Pl. 25, Figs. 9, 10.

Globigerinoides primordius Blow and Banner

(Plate 2, Figs. 12 and 13)

Globigerinoides primordius Blow and Banner, 1962.

Globigerinoides quadrilobatus (d'Orbigny) s.1.

(Plate 2, Figs. 15-19)

Globigerina quadrilobata d'Orbigny, 1846, p. 164, Pl. 9, Figs. 7-10. Globigerina sacculifera Brady, 1877, p. 535; 1884, Pl. 80, Figs. 11-17. Globigerina sacculiferus (Brady) var. immatura LeRoy, 1939, p. 263, Pl. 3, Figs. 19-21.

Globigerina triloba Reuss, 1850, p. 374, Pl. 447, Fig. 11.

Remarks. The four subspecies G. quadrilobatus immaturus, G. quadrilobatus trilobus, G. quadrilobatus quadrilobatus, and G. quadrilobatus sacculifer were not separated in the species examination for this report.

Globigerinoides ruber (d'Orbigny) s.1.

(Plate 2, Figs. 20 and 21)

Globigerina rubra d'Orbigny, 1839, p. 82, Pl. 4, Figs. 12-14.

Globigerinoides subquadratus Brönnimann, in Todd et al., 1954, p. 680 , Pl. 1, Figs. 5 and 8.

Remarks. The early-middle Miocene species $G$. subquadratus was not separated in this report from the late middle Miocene to Holocene species $G$. ruber. 
Table 3. Distribution of Neogene planktonic foraminifers in sediments of Hole 705A.

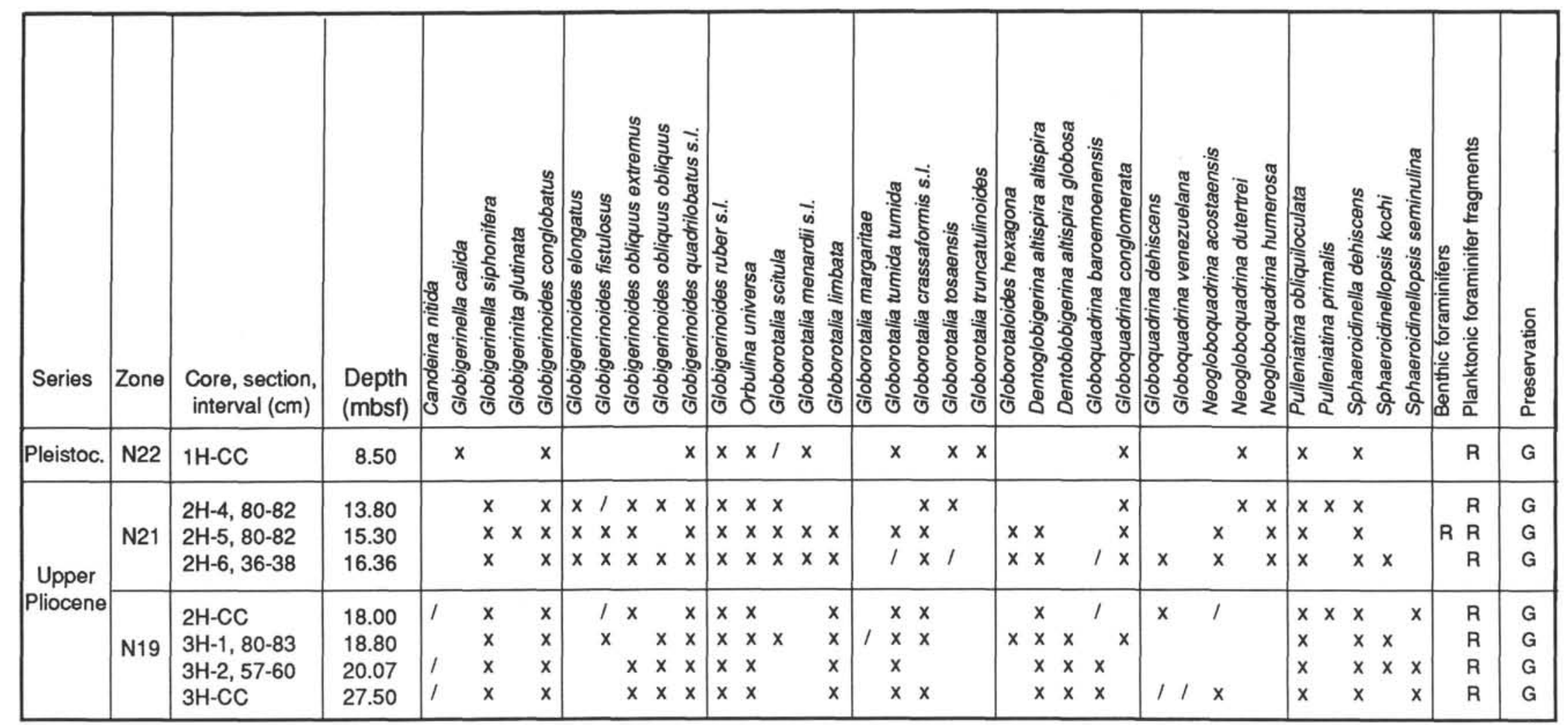


Table 4. Distribution of Neogene planktonic foraminifers in sediments of Hole 706A.

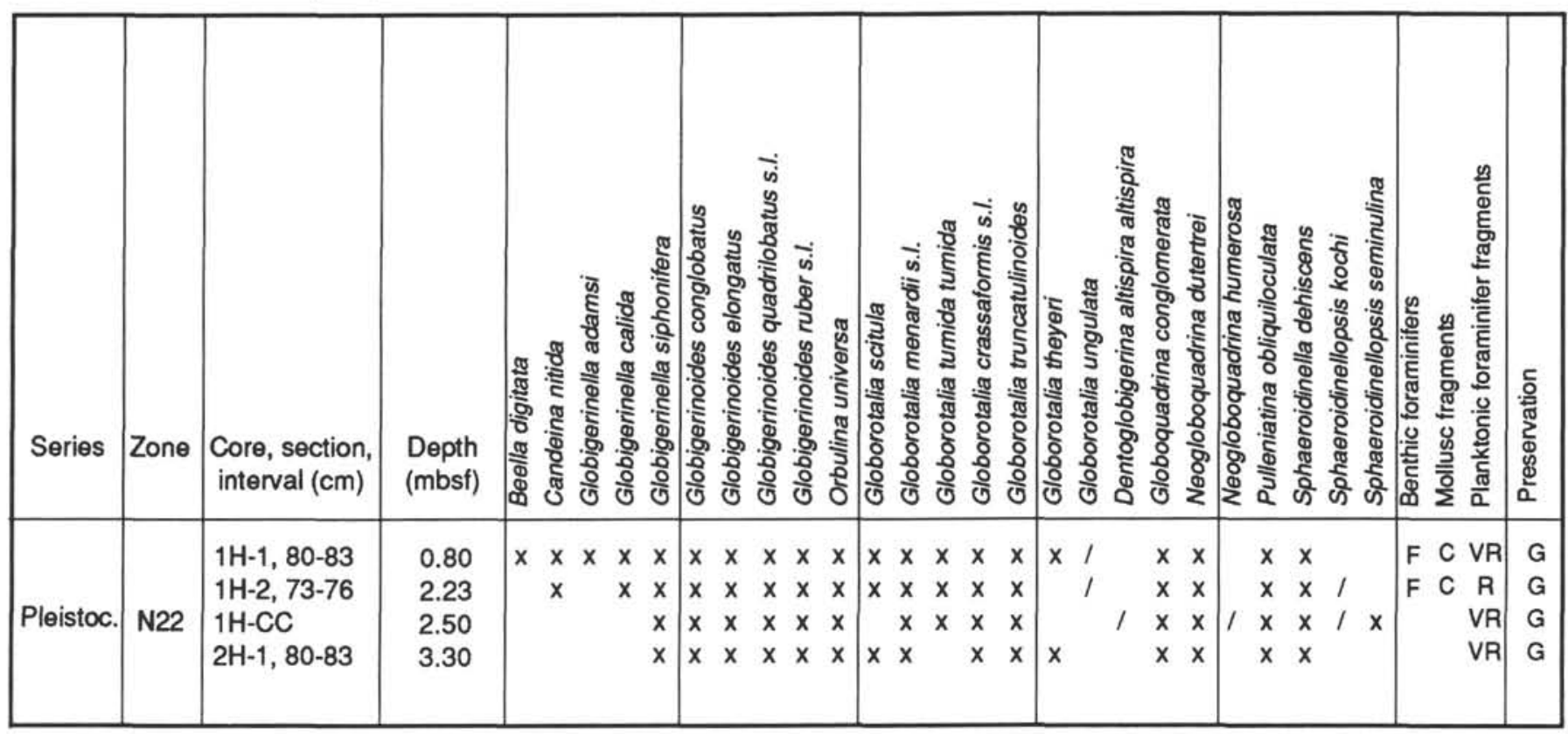

Globoquadrina baroemoenensis (LeRoy)

(Plate 6, Figs. 11 and 16)

Globigerina baroemoenensis LeRoy, 1939, p. 263, Pl. 6, Figs. 1 and 2.

\section{Globoquadrina binaiensis (Koch)}

(Plate 6, Figs. 12-15)

Globigerina? aspera Koch, 1926, p. 746, Fig. 22.

Globigerina binaiensis Koch, 1935, p. 558.

\section{Globoquadrina conglomerata (Schwager)}

(Plate 6, Fig. 21)

Globigerina conglomerata Schwager, 1866, Pl. 7, Fig. 113.

Globigerina conglomerata Schwager, Banner and Blow, 1960, Pl. 2, Fig. 3 (neotype).

Globoquadrina conglomerata (Schwager), Parker, 1962, Pl. 6, Figs. 1118.

Globoquadrina dehiscens (Chapman, Parr and Collins)

(Plate 6, Fig. 17)

Globorotalia dehiscens Chapman, Parr and Collins, 1934, p. 569, Pl. 11, Fig. 36.

\section{Globoquadrina praedehiscens Blow and Banner} (Plate 6, Figs. 18 and 19)

Globoquadrina dehiscens praedehiscens Blow and Banner, 1962, p. 116, Pl. 15, Figs. Q-S.

Globoquadrina sellii Borsetti

(Plate 6, Figs. 20 and 24)

Globoquadrina sellii Borsetti, 1959, Pl. 1, Fig. 3.

Globoquadrina tripartita (Koch)

Globigerina bulloides var. tripartita Koch, 1926, p. 746, Fig. 21.

Globoquadrina venezuelana (Hedberg)

(Plate 6, Figs. 22 and 23)

Globigerina venezuelana Hedberg, 1937, p. 681, Pl. 92, Fig. 7.
Globorotalia archaeomenardii Bolli

Globorotalia archaeomenardii Bolli, 1957, p. 119, Pl. 28, Fig. 11.

Globorotalia crassaformis (Galloway and Wissler) (Plate 5, Fig. 11)

Globigerina crassaformis Galloway and Wissler, 1927, p. 41, Pl. 7, Fig. 12

Globorotalia fohsi fohsi Cushman and Ellisor (Plate 4, Figs. 4-6)

Globorotalia fohsi Cushman and Ellisor, 1939, p. 12, Pl. 2, Fig. 6.

Globorotalia fohsi lobata Bermudez (Plate 4, Figs. 7-9)

Globorotalia lobata Bermudez, 1949, p. 286, Pl. 22, Figs. 15-17.

Globorotalia fohsi robusta Bolli

Globorotalia fohsi robusta Bolli, 1950, pp. 84, 89, Pl. 15, Fig. 3.

Globorotalia inflata d'Orbigny

Globigerina inflata d'Orbigny, 1839, in Barker-Webb, P., and Berthelot, S., 1839, p. 134, Pl. 2, Figs. 7-9.

Globorotalia juanai Bermudez and Bolli

Globorotalia juanai Bermudez and Bolli, 1969, pp. 171-172, Pl. 14, Figs. 1-6.

\section{Globorotalia kugleri Bolli}

(Plate 3, Figs. 8-11)

Globorotalia kugleri Bolli, 1957, p. 118, Pl. 28, Fig. 5.

Globorotalia limbata (d'Orbigny)

(Plate 4, Figs. 19-21 and 24-28)

Rotalia limbata d'Orbigny, 1826, p. 274 (nomen nudum).

Rotalia limbata d'Orbigny, in Fornasini, 1902, p. 56, Fig. 55.

Rotalia limbata d'Orbigny, Banner and Blow, 1960, pp. 30-31, Pl. 5, Fig. 3 (lectotype). 
Globorotalia (Globorotalia) cultrata limbata (d'Orbigny), Blow, 1969, Pl. 7, Figs. 4-6; PI. 42, Figs. 2, 3.

Globorotalia multicamerata Cushman and Jarvis, Lamb and Beard, 1972, Pl. 14, Figs. 5-8.

Globorotalia margaritae Bolli and Bermudez

(Plate 5, Figs. 4-6)

Globorotalia margaritae Bolli and Bermudez, 1965, p. 138, PI. 1, Figs. 16-18.

Globorotalia mayeri Cushman and Ellisor-Globorotalia siakensis LeRoy group

(Plate 3, Figs. 12-20)

Globorotalia mayeri Cushman and Ellisor, 1939, p. 11, Pl. 2, Fig. 4. Globorotalia siakensis LeRoy, 1939, p. 262.

Remarks. No attempt was made in this report to separate the two species $G$. mayeri and $G$. siakensis.

Globorotalia menardii (Parker, Jones and Brady) s.1. (Plate 4, Figs. 10-18, 22, and 23)

Rotalia menardii Parker, Jones and Brady, 1865, p. 20, Pl. 3, Fig. 81. Pulvinulina menardii (d'Orbigny) var. fimbriata Brady, 1884, p. 691, Pl. 103, Fig. 3.

Remarks. The Pleistocene variant fimbriata characterized by a peripheral carina with spikelike outgrowth (see Plate 7, Figs. 17, 18, 22, and 23) was not treated as a subspecies in this report.

Globorotalia miozea Finlay

(Plate 5, Figs. 1-3)

Globorotalia miozea Finlay, 1939, p. 326, Pl. 29, Figs. 159-161.

Globorotalia opima nana Bolli

Globorotalia opima nana Bolli, 1957, p. 118, Pl. 28, Fig. 3.

Globorotalia peripheroacuta Blow and Banner

(Plate 3, Figs. 26-28)

Globorotalia (Turborotalia) peripheroacuta, Blow and Banner, 1966, p. 294, Pl. 1, Fig. 2.

Globorotalia peripheroronda Blow and Banner (Plate 3, Figs. 23-25)

Globorotalia (Turborotalia) peripheroronda Blow and Banner, 1966, p. 294, Pl. 1, Fig. 1.

Globorotalia plesiotumida Blow and Banner

Globorotalia (G.) tumida (Brady) plesiotumida Blow and Banner, 1965, p. 1353, Fig. 2.

Globorotalia praefohsi Blow and Banner

(Plate 4, Figs. 1-3)

Globorotalia (Globorotalia) praefohsi Blow and Banner, 1966, p. 295 , Pl. 1, Figs. 3-4; Pl. 2, Figs. 6, 7 and 10-11.

Globorotalia praemenardii Cushman and Stainforth

Globorotalia praemenardii Cushman and Stainforth, 1945, p. 70, Pl 13, Fig. 14.

\section{Globorotalia praescitula Blow}

Globorotalia scitula praescitula Blow, 1959, p. 221, Pl. 19, Fig. 12.

Globorotalia pseudokugleri Blow

(Plate 3, Figs. 6-7)

Globorotalia pseudokugleri Blow, 1969, p. 391.
Globorotalia scitula (Brady)

(Plate 3, Figs. 21, 22, and 29)

Pulvinulina scitula Brady, 1882, p. 716 (figures in Brady, 1882, Pl. 103, Fig. 7).

Pulvinulina scitula Brady, Banner and Blow, 1960, Pl. 5, Fig. 5 (lectotype).

Globorotalia theyeri Fleisher (Plate 5, Figs. 13-15)

Globorotalia theyeri Fleisher, 1974, p. 1028, Pl. 12, Fig. 9; Pl. 13, Figs. $1-5$.

Globorotalia tosaensis Takayanagi and Saito

Globorotalia tosaensis Takayanagi and Saito, 1962, p. 81, Pl. 28, Figs. 11-12.

Globorotalia truncatulinoides (d'Orbigny)

(Plate 5, Figs. 12, 18, and 23)

Rotalina truncatulinoides d'Orbigny, 1839, in Barker-Webb, P., and Berthelot, S., p. 132, Pl. 2, Figs. 25-27.

Globorotalia tumida tumida (Brady)

(Plate 5, Fig. 7)

Pulvinulina menardii (d’Orbigny) var. tumida Brady, 1877, p. 535.

Globorotalia tumida flexuosa (Kock)

(Plate 5, Figs. 8-10)

Pulvinulina tumida Brady var. flexuosa Kock, 1923, p. 351, Figs. 9 and 10.

Globorotalia ungulata Bermudez (Plate 5, Figs. 16 and 17)

Globorotalia ungulata Bermudez, 1960, p. 1304, Pl. 15, Fig. 6.

Globorotaloides hexagona (Natland)

(Plate 5, Figs. 19, 20, and 24)

Globigerina hexagona Natland, 1938, p. 149, Pl. 7, Fig. 1.

Globorotaloides suteri Bolli

(Plate 5, Figs. 21 and 22)

Globorotaloides suteri Bolli, 1957, p. 117, Pl. 27, Figs. 9-13.

Globorotaloides variabilis Bolli

(Plate 5, Figs. 25-27)

Globorotaloides variabilis Bolli, 1957, p. 117, Pl. 27, Figs. 15-20.

Globoturborotalita druryi (Akers)

Globigerina druryi Akers, 1955, p. 654, Pl. 65, Fig. 1.

\section{Globoturborotalita nepenthes (Todd)}

(Plate 7, Figs. 1 and 2)

Globigerina nepenthes Todd, 1957, p. 301, Fig. 7.

Neogloboquadrina acostaensis (Blow)

(Plate 7, Figs. 3-6, 10, and 11)

Globorotalia acostaensis Blow, 1959, p. 208, PI. 17, Fig. 106.

Neogloboquadrina dutertrei (d'Orbigny)

(Plate 7, Figs. 12-16)

Globigerina dutertrei d'Orbigny, 1839, p. 84, Pl. 4, Figs. 19-21. 
Table 5. Distribution of Neogene planktonic foraminifers in sediments of Hole $707 \mathrm{~A}$.

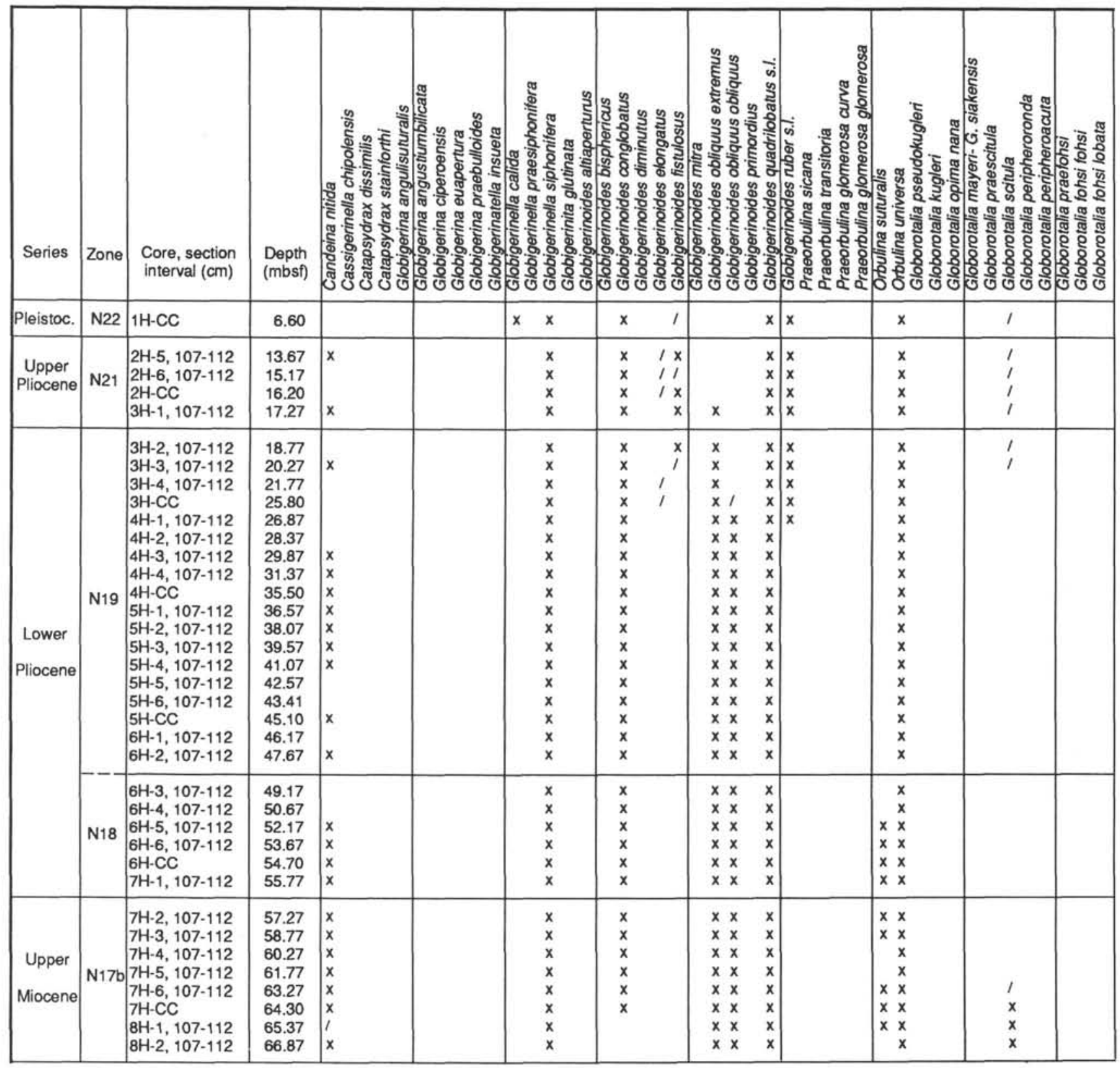

Neogloboquadrina humerosa (Takayanagi and Saito) (Plate 7, Figs. 7-9)

Globorotalia humerosa Takayanagi and Saito, 1962, p. 78, Pl. 28, Figs. $1-2$.

Orbulina suturalis Brönnimann

(Plate 3, Figs. 4 and 5)

Orbulina suturalis Brönnimann, 1951, p. 135, Fig. IV, Figs. 15, 16, and 20 .

Orbulina universa d'Orbigny

Orbulina universa d'Orbigny, 1839, p. 3, Pl. 1, Fig. 1.

\section{Praeorbulina glomerosa circularis (Blow)} (Plate 3, Fig. 3)

Globigerinoides glomerosa circularis Blow, 1956, p. 65, Fig. 2, nos. 3-4.
Praeorbulina glomerosa curva (Blow)

(Plate 2, Figs. 25 and 26)

Globigerinoides glomerosa curva Blow, 1956, p. 64, Fig. 1, nos. 9-14.

Praeorbulina glomerosa glomerosa (Blow)

(Plate 3, Figs. 1-2)

Globigerinoides glomerosa glomerosa Blow, 1956, p. 64, Fig. 1, nos. 15-19, Fig. 2, nos. 1-2.

Praeorbulina sicana (de Stefani)

(Plate 2, Figs. 22-24)

Globigerinoides sicana de Stefani, 1952, p. 9 (type figure designated as Globigerinoides conglobata of Cushman and Stainforth, 1945, p. 68, Pl. 13, Fig. 6).

Praeorbulina transitoria (Blow)

Globigerinoides transitoria Blow, 1956, p. 65, Fig. 2, nos. 12-13. 
Table 5 (Continued).

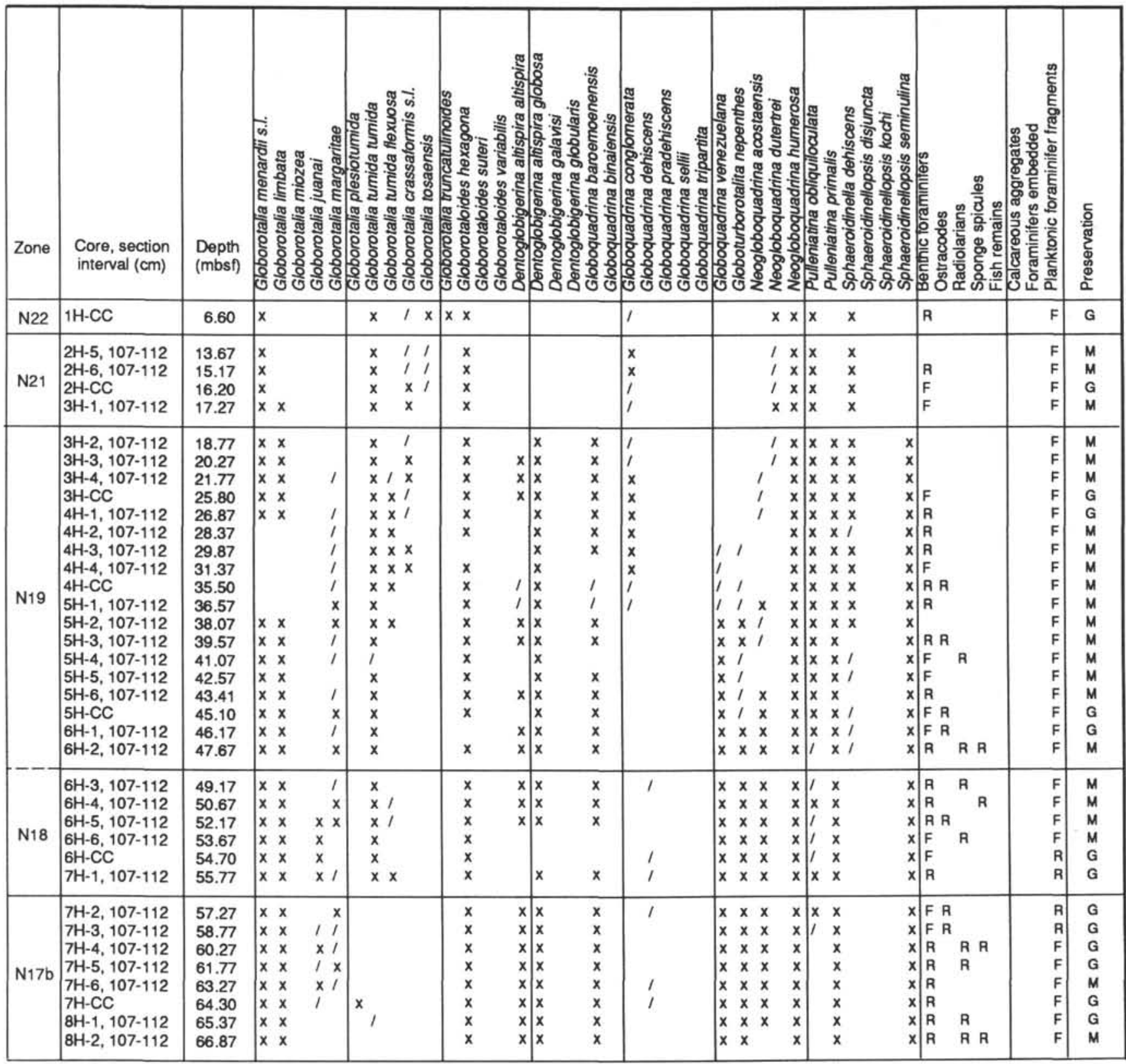

Pulleniatina obliquiloculata (Parker and Jones)

(Plate 7, Fig. 20)

Pullenia sphaeroides (d'Orbigny) var. obliquiloculata Parker and Jones; 1865 , p. 368 , Pl, 19, Fig. 4.

Pulleniatina primalis Banner and Blow (Plate 7, Figs. 17-19)

Pulleniatina primalis Banner and Blow, 1967, p. 142, Pl. 1, Figs. 3-8; Pl. 3, Fig. 2.

Sphaeroidinella dehiscens (Parker and Jones) (Plate 7, Figs. 21 and 22)

Sphaeroidina bulloides d'Orbigny var. dehiscens Parker and Jones, 1865, p. 369 , Pl. 19, Fig. 5 .

\section{Sphaeroidinellopsis disjuncta (Finlay)}

Sphaeroidinella disjuncta Finlay, 1940, p. 467, Pl. 67, Figs. 224-228.

\section{Sphaeroidinellopsis kochi Caudri}

(Plate 7, Figs. 23-26)

Globigerina kochi Caudri, 1934, p. 144 (type figures in Eclogae Geol. Helv., Bd. 18(2), Fig. 8).

Sphaeroidinella multiloba LeRoy, 1944, p. 91, Pl. 4, Figs. 7-9.

Sphaeroidinella seminulina (Schwager), Parker, 1967, p. 161, Pl. 23, Figs. 1-5.

Sphaeroidinellopsis hanckocki Bandy, 1975, p. 57, Pl. 1, Fig. 3.

\section{Sphaeroidinellopsis seminulina (Schwager)}

Globigerina seminulina Schwager, 1866, p. 256, Pl. 7, Fig. 112.

Sphaeroidinella dehiscens subdehiscens Blow, 1959, p. 195, Pl. 12, Figs. 71-72.

Sphaeroidinella subdehiscens Blow, Parker, 1967, p. 162, Pl. 23, Figs. 6-7. 
Table 5 (Continued).

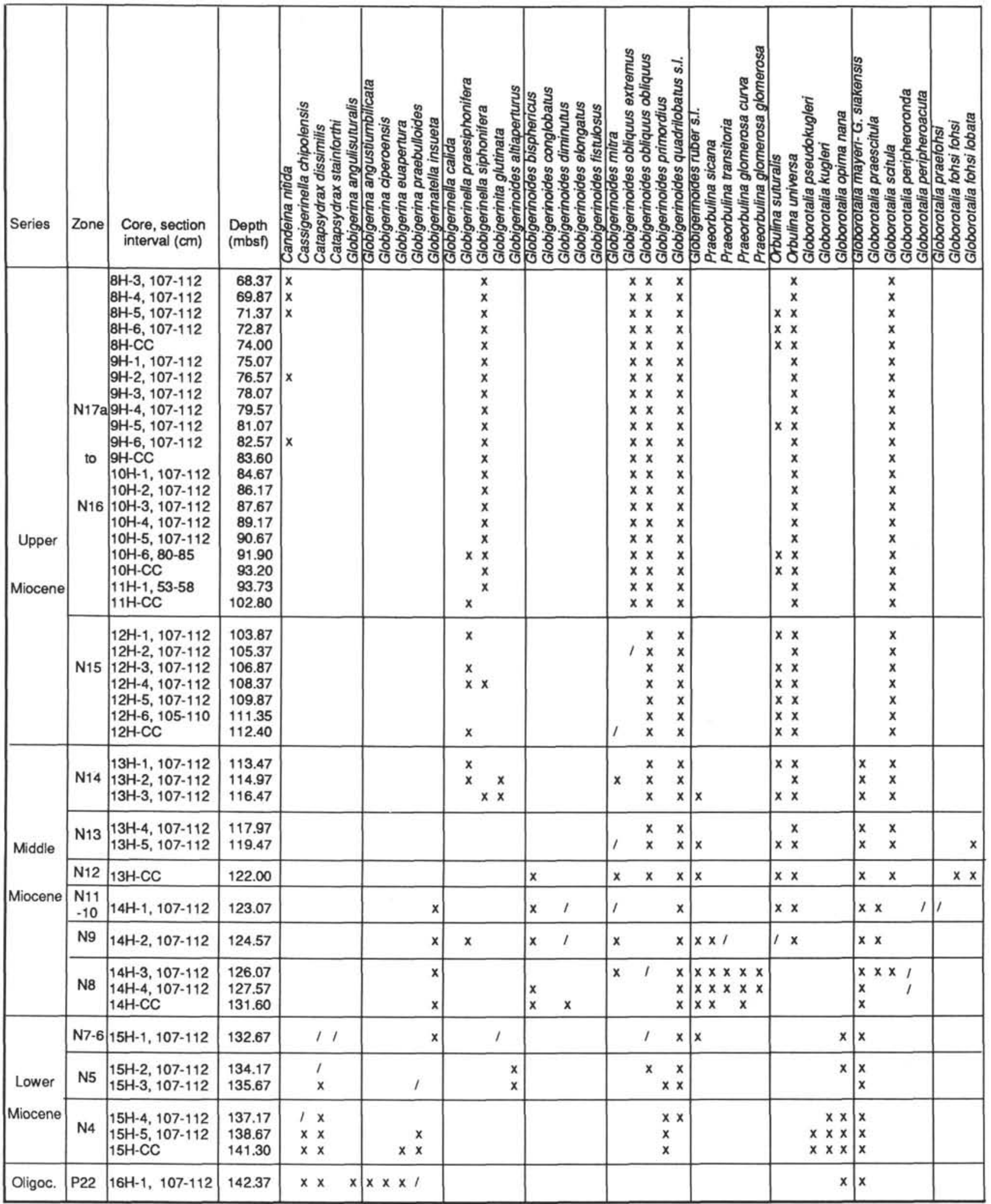


Table 5 (Continued).

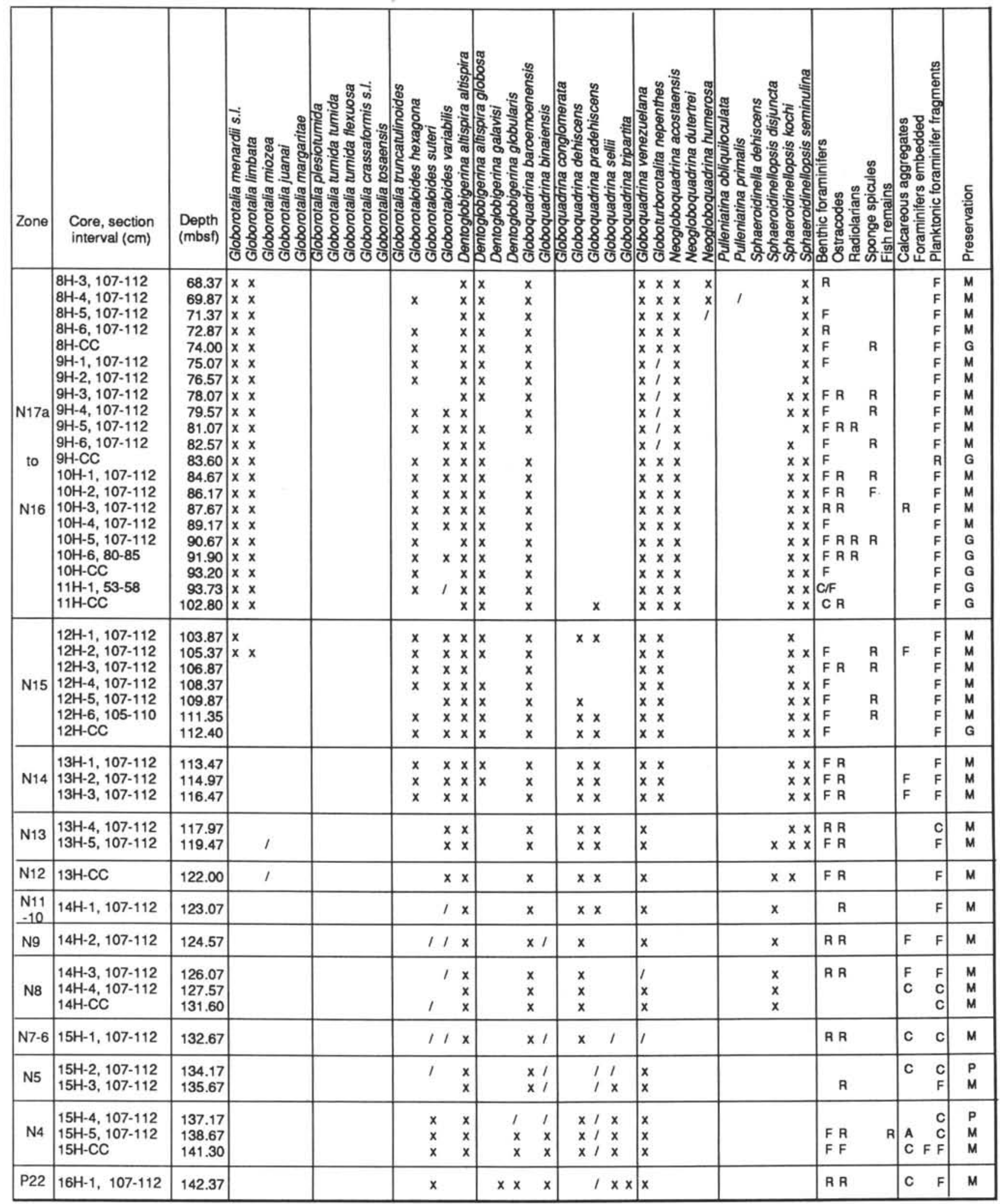


Table 6. Distribution of Neogene planktonic foraminifers in sediments of Hole 708A.

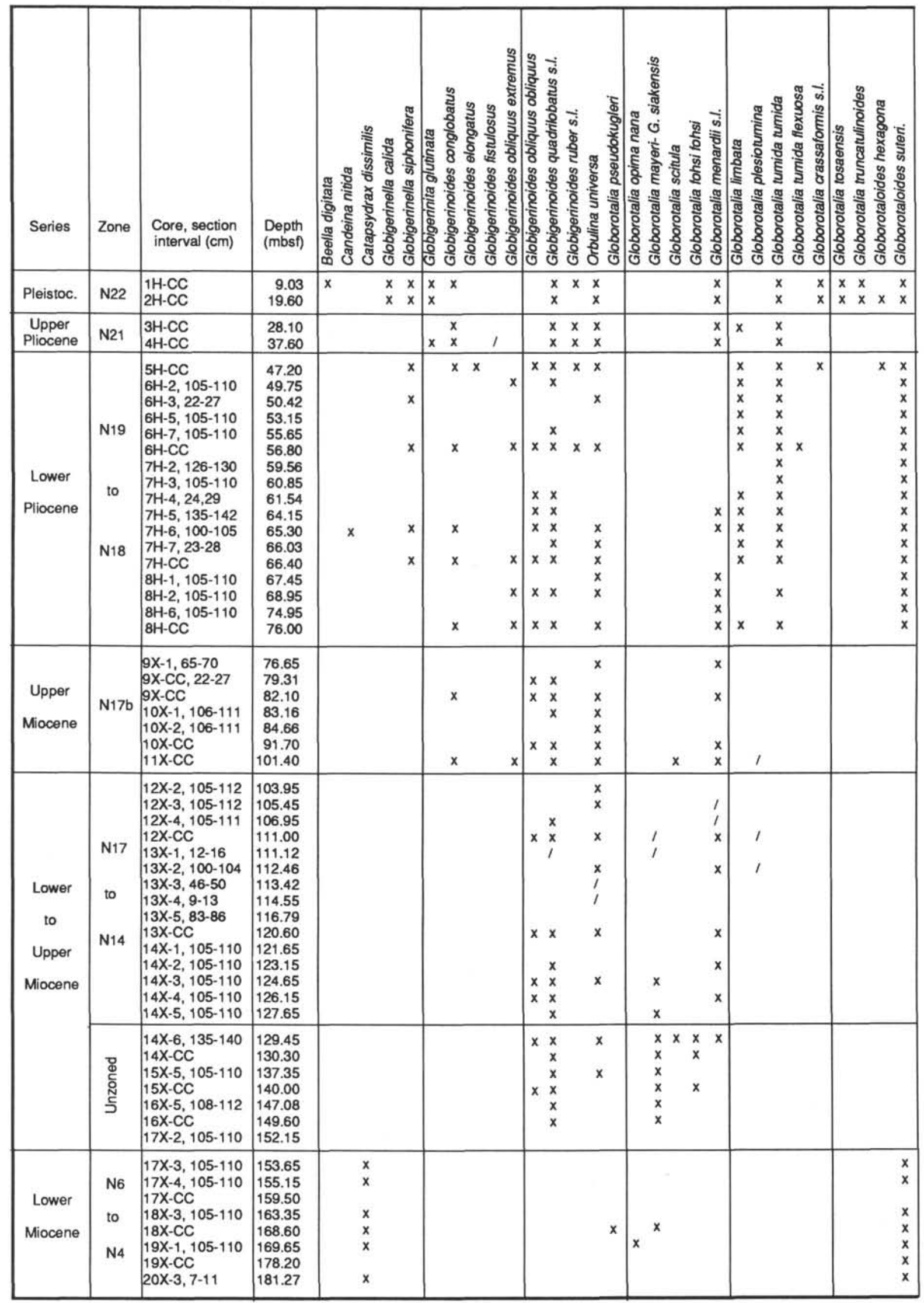


Table 6 (Continued).

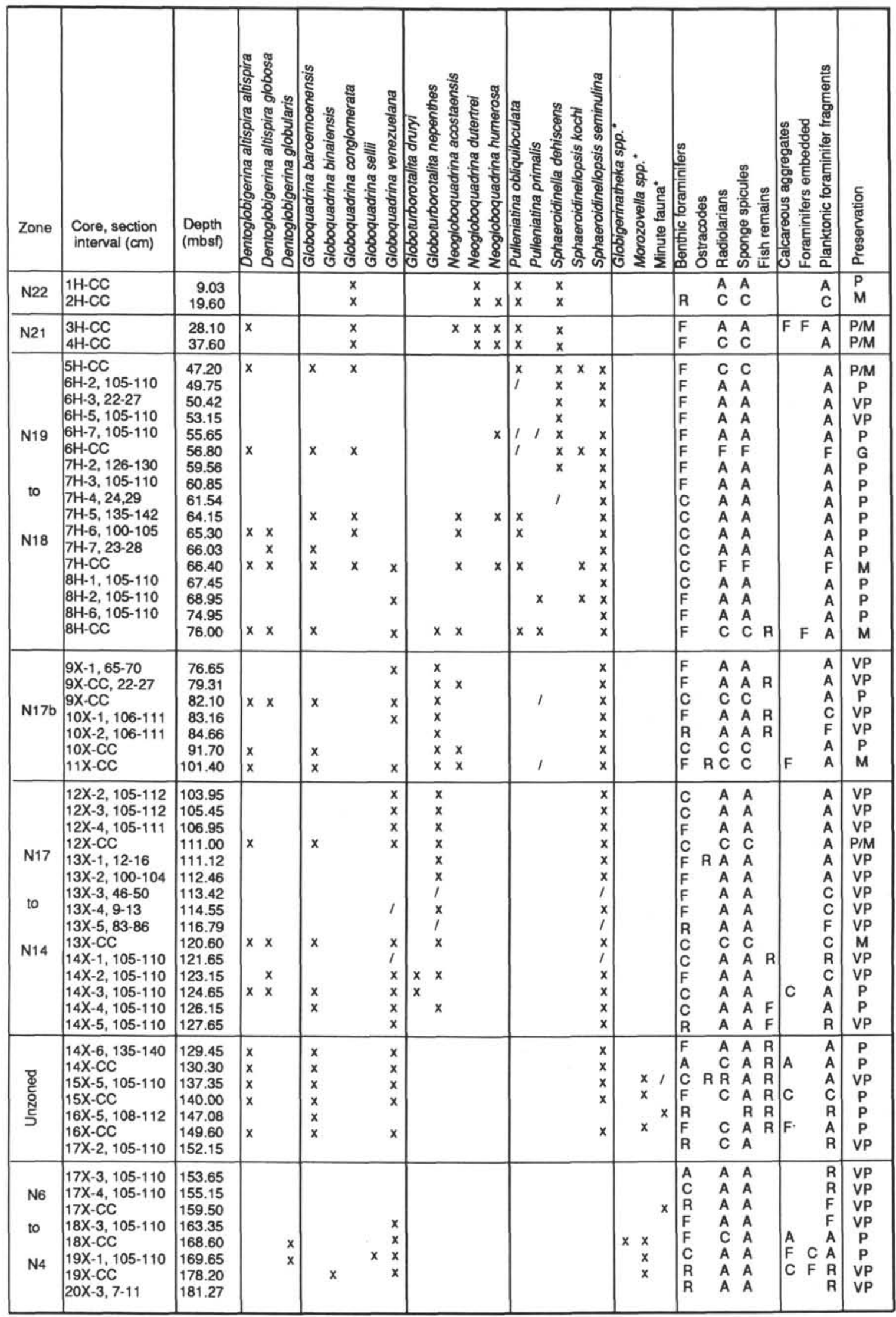

- Reworked 
Table 7. Distribution of Neogene planktonic foraminifers in sediments of Hole 709C.

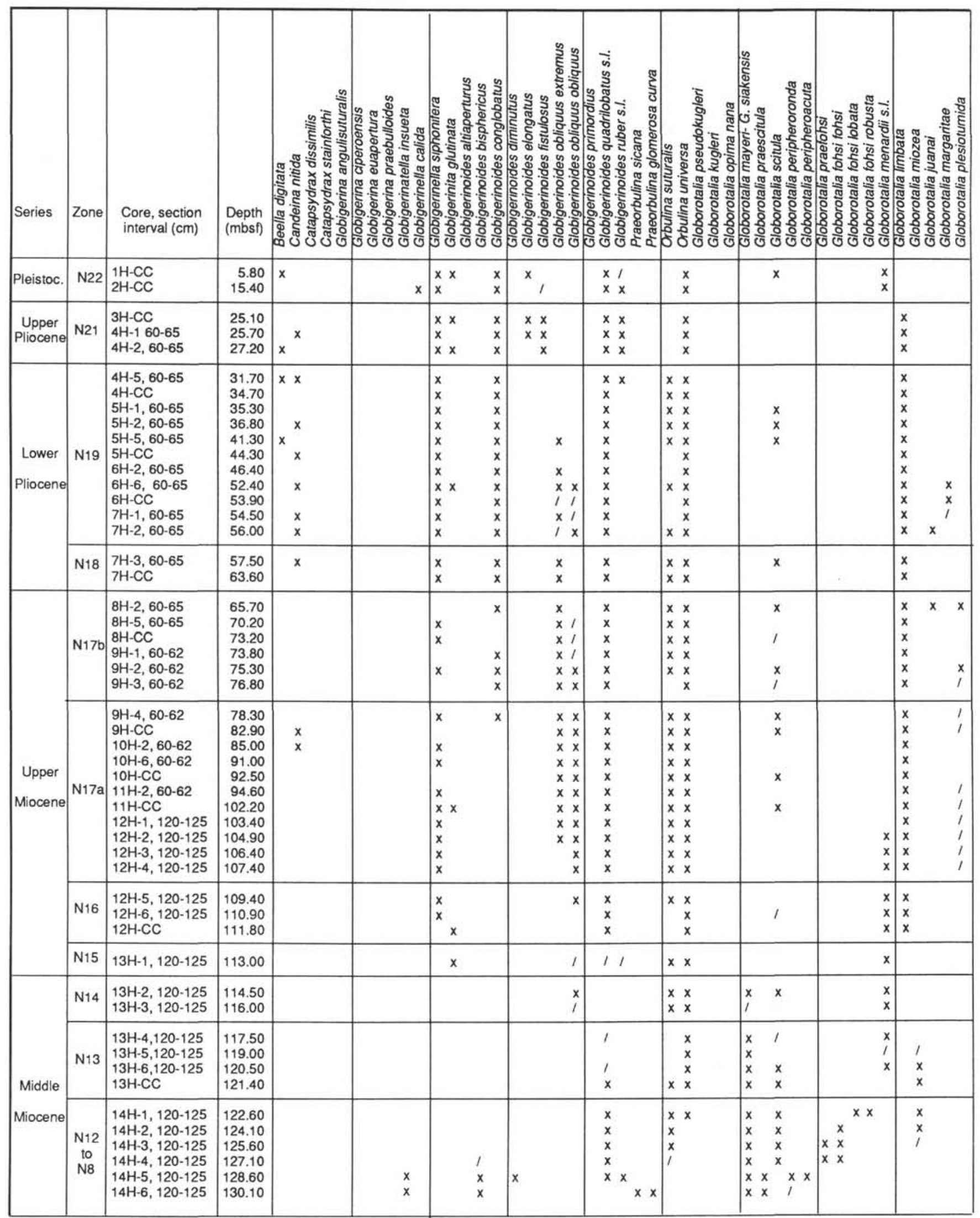


Table 7 (Continued).

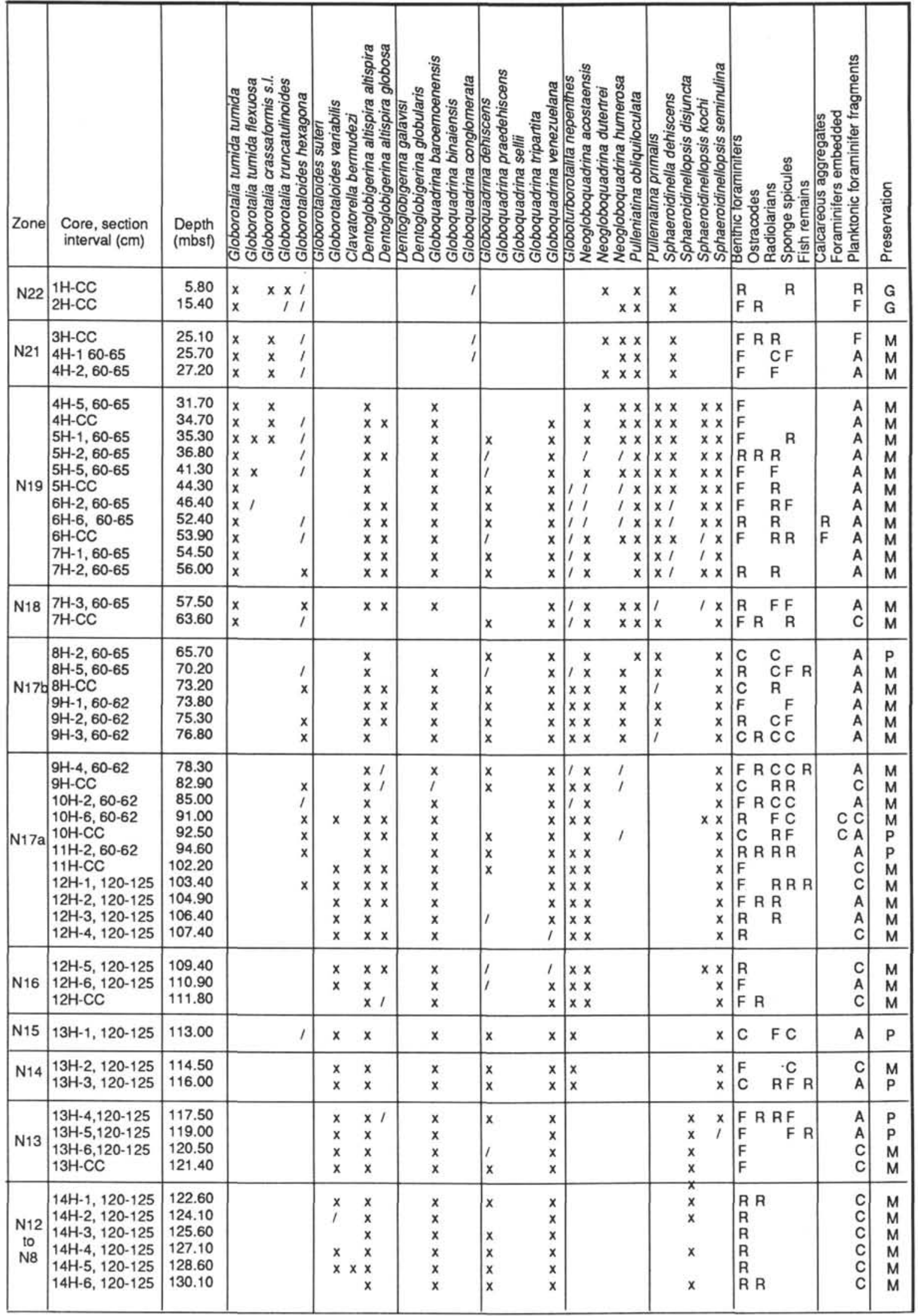


Table 7 (Continued).

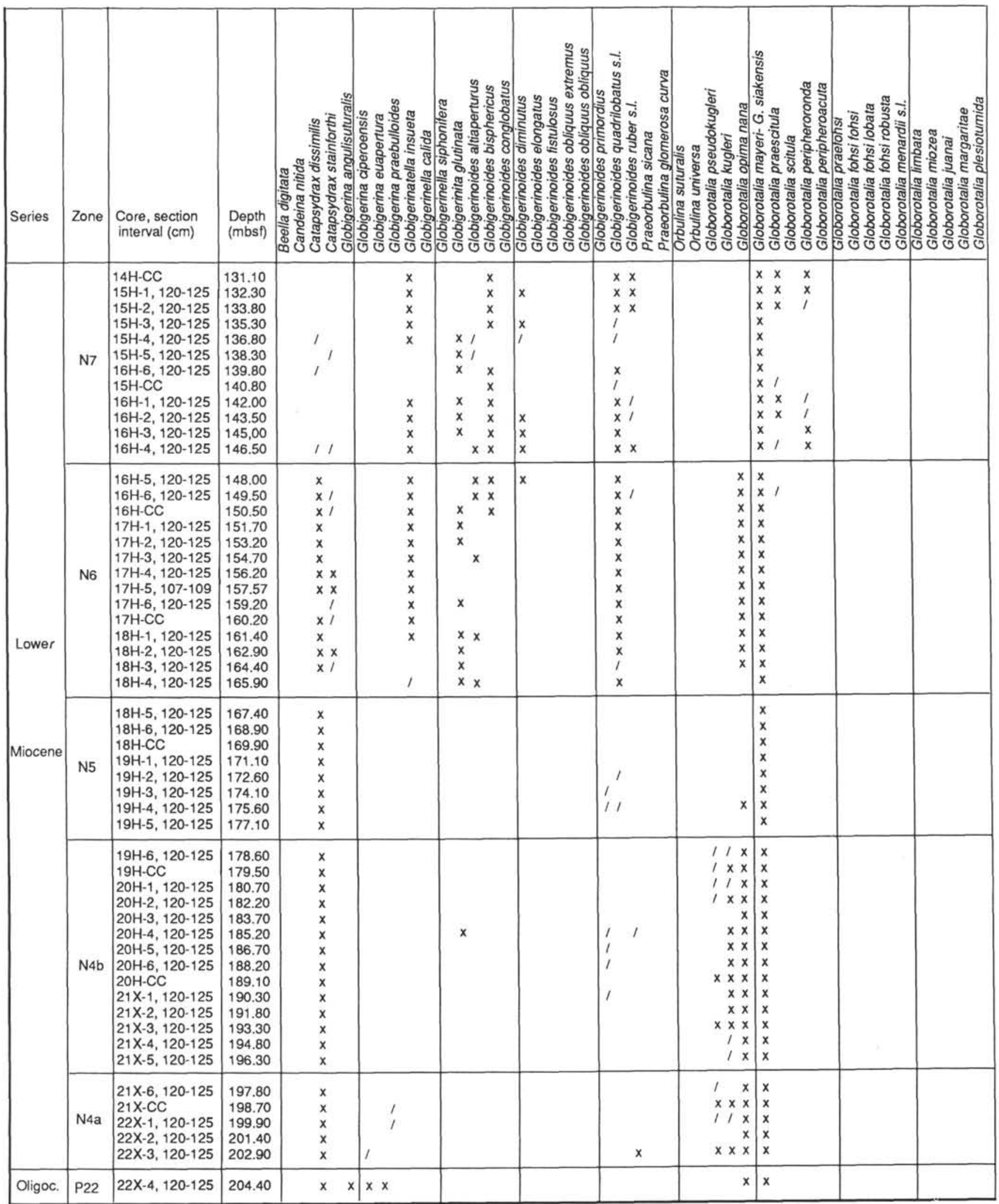


Table 7 (Continued).

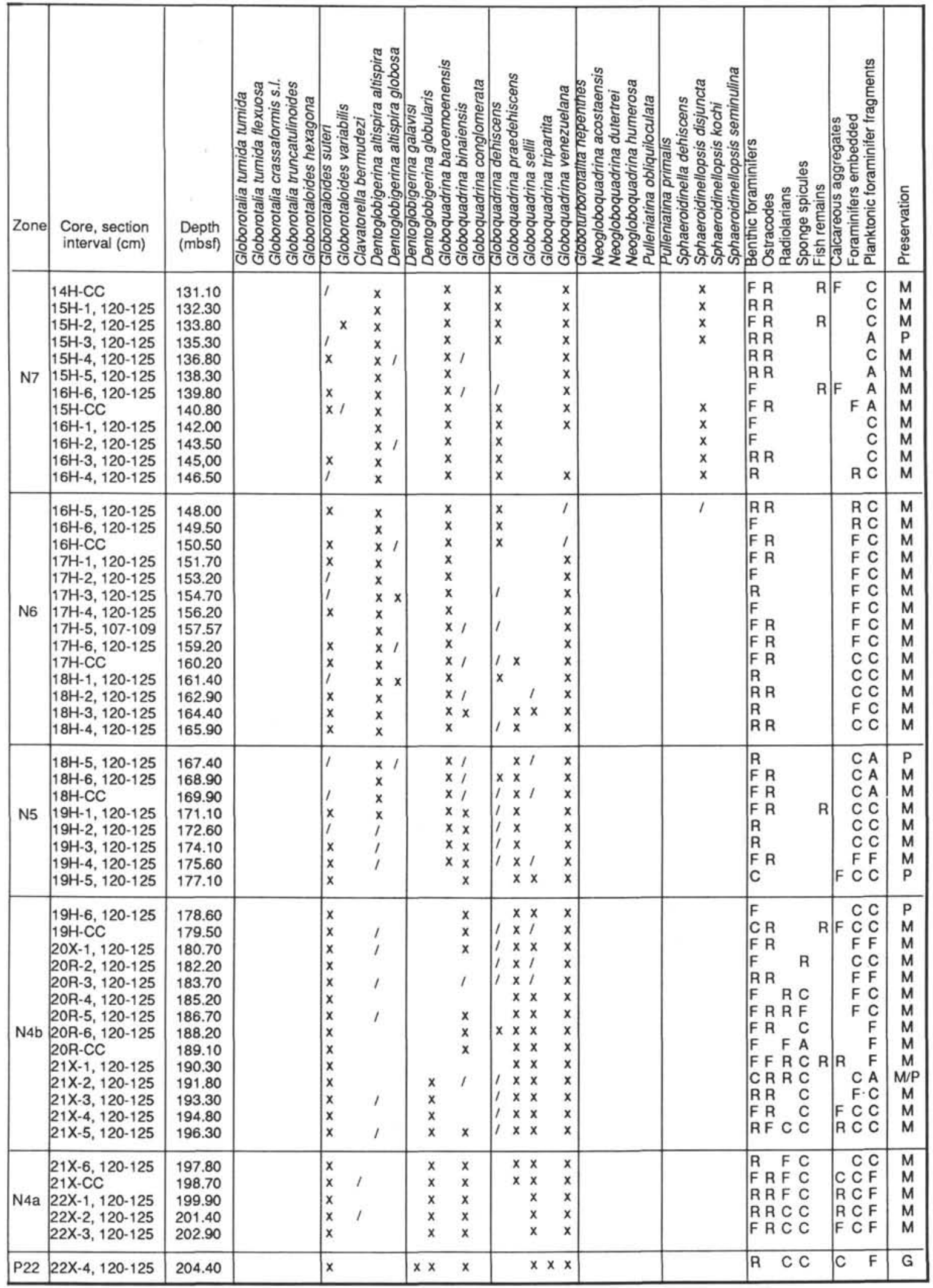




\begin{tabular}{|c|c|c|c|c|c|c|c|c|c|c|c|c|c|c|c|c|c|}
\hline Series & Zone & $\begin{array}{l}\text { Core, section } \\
\text { interval (cm) }\end{array}$ & $\begin{array}{l}\text { Depth } \\
\text { (mbsf) }\end{array}$ & 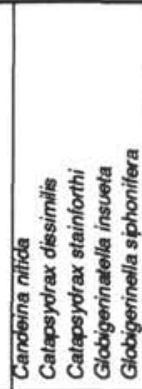 & 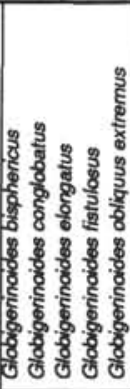 & 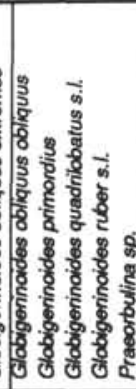 & 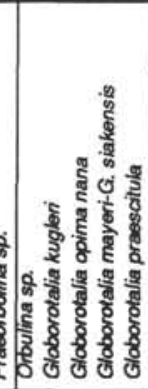 & 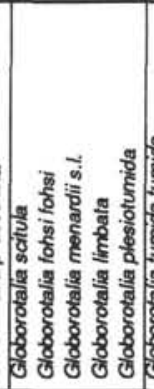 & 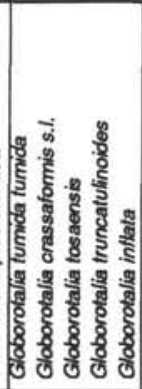 & 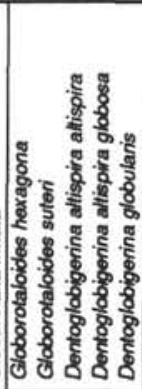 & 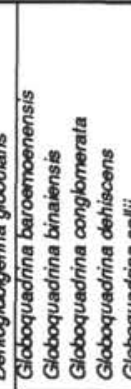 & 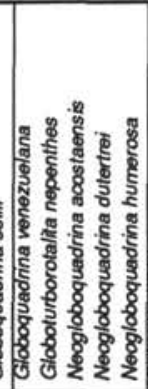 & 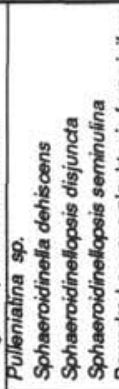 & 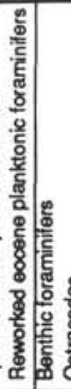 & 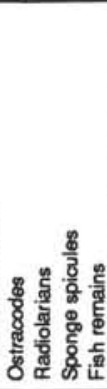 & 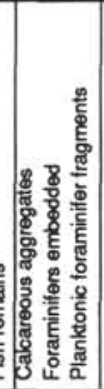 & 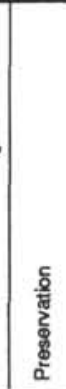 \\
\hline Pleistoc. & N22 & $\begin{array}{l}1 \mathrm{H}-1,105-110 \\
1 \mathrm{H}-2,90-95 \\
1 \mathrm{H}-3,105-110 \\
1 \mathrm{H}-4,105-110 \\
1 \mathrm{H}-5,105-110 \\
\end{array}$ & $\begin{array}{l}1.05 \\
2.40 \\
4.05 \\
5.55 \\
7.05 \\
\end{array}$ & $\begin{array}{ll} & \mathrm{x} \\
& \\
& \\
& \mathrm{x} \\
& \mathrm{x}\end{array}$ & $\begin{array}{l}x \\
x \\
x \\
x \\
x\end{array}$ & $\begin{array}{ll}x \\
x \\
1 \\
x & x \\
x & x \\
\end{array}$ & $\begin{array}{l}1 \\
1 \\
x \\
x\end{array}$ & $\begin{array}{|ll|} & \mathrm{x} \\
\times & \mathrm{x} \\
& \mathrm{x} \\
\times & \mathrm{x} \\
& \mathrm{x} \\
\end{array}$ & $\begin{array}{lllll}x & x & l \\
x & x & & \\
x & x & x & \\
x & x & & x & x \\
x & x & x & x & x\end{array}$ & $\begin{array}{l}x \\
x\end{array}$ & $\begin{array}{l}x \\
x \\
x\end{array}$ & \begin{tabular}{|l|l|}
1 & $x$ \\
$x$ \\
$x$ \\
$x$ & $x$ \\
$x$ & $x$ \\
\end{tabular} & $\begin{array}{|ll|}x & x \\
x & \\
x & x \\
x & x \\
x & x \\
\end{array}$ & $\begin{array}{l}F \\
F \\
F / C \\
R \\
F\end{array}$ & 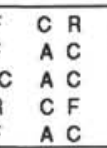 & \begin{tabular}{l|}
$A$ \\
$C$ \\
$A$ \\
$A$ \\
$A$
\end{tabular} & $\begin{array}{l}M \\
M \\
\text { VP } \\
G \\
M\end{array}$ \\
\hline $\begin{array}{l}\text { Upper } \\
\text { Pliocene }\end{array}$ & N21 & $\begin{array}{l}1 H-6,105-110 \\
1 H-C C \\
2 H-1,105-110 \\
2 H-2,105-110 \\
2 H-3,105-110 \\
2 H-4,105-110 \\
2 H-5,105-110 \\
2 H-6,105-110 \\
2 H-C C \\
3 H-1,105-110 \\
3 H-2,105-110 \\
3 H-3,105-110 \\
\end{array}$ & \begin{tabular}{|r|}
8.55 \\
9.50 \\
10.55 \\
12.05 \\
13.55 \\
15.05 \\
16.55 \\
18.05 \\
19.20 \\
20.25 \\
21.75 \\
23.25 \\
\end{tabular} & $\begin{array}{l}x \\
x \\
x \\
x\end{array}$ & $\begin{array}{ll}\mathrm{x} & \\
\mathrm{x} & \\
\mathrm{x} & 1 \\
\mathrm{x} & \\
\mathrm{x} & \\
\mathrm{x} & \mathrm{x} \\
\mathrm{x} & \mathrm{x} \\
\mathrm{x} & \\
\mathrm{x} & \mathrm{x} \\
\mathrm{x} & \\
1 & \\
& \\
\end{array}$ & $\begin{array}{ll}x & \\
x & x \\
x & x \\
x & x \\
x & x \\
x & x \\
x & x \\
x & \\
x & x \\
x & \\
t & \\
l & x \\
\end{array}$ & $\begin{array}{l}x \\
x \\
x \\
x \\
x \\
x \\
x \\
x \\
x \\
x \\
x \\
x\end{array}$ & 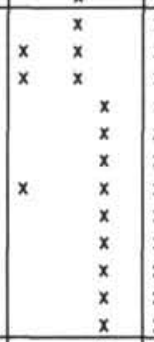 & $\begin{array}{|lll|}x & x & 1 \\
x & x & x \\
x & & 1 \\
x & & 1 \\
x & x & \\
x & & \\
x & x & \\
x & x & \\
x & x & \\
x & x & \\
x & x & \\
x & x & \\
\end{array}$ & $\begin{array}{l}x \\
x \\
x \\
x\end{array}$ & I & $\begin{array}{ll}x & x \\
x & x \\
x & x \\
x & x \\
x & x \\
x & x \\
1 & x \\
1 & x \\
1 & x \\
& 1 \\
& \\
\end{array}$ & $\begin{array}{ll}x & x \\
x & x \\
x & x \\
x & x \\
x & x \\
x & x \\
x & x \\
x & x \\
x & x \\
1 & x \\
1 & x \\
1 & x \\
\end{array}$ & $\begin{array}{l}F \\
F \\
F \\
R \\
R \\
R \\
R \\
F \\
R \\
C \\
C \\
C\end{array}$ & 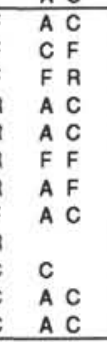 & $\begin{array}{l}A \\
A \\
A \\
A \\
A \\
A \\
A \\
A \\
F \\
A \\
A \\
A\end{array}$ & $\begin{array}{l}M \\
G \\
M \\
\text { VP } \\
\text { VP } \\
M \\
M \\
M \\
G \\
P \\
\text { VP } \\
P\end{array}$ \\
\hline $\begin{array}{l}\text { Lower } \\
\text { Pliocene }\end{array}$ & $\begin{array}{l}\text { N19 } \\
\text { to } \\
\text { N18 }\end{array}$ & $\begin{array}{l}3 \mathrm{H}-\mathrm{CC} \\
4 \mathrm{H}-1,105-110 \\
4 \mathrm{H}-2,105-110 \\
4 \mathrm{H}-3,105-110 \\
4 \mathrm{H}-4,105-110 \\
4 \mathrm{H}-5,105-110 \\
4 \mathrm{H}-6,105-110 \\
4 \mathrm{H}-\mathrm{CC}\end{array}$ & \begin{tabular}{l|}
28.80 \\
29.85 \\
31.35 \\
32.85 \\
34.35 \\
35.85 \\
37.35 \\
38.30
\end{tabular} & 1 & $\times 1$ & $\begin{array}{l}1 \\
1 \\
1\end{array}$ & $\begin{array}{l}x \\
x \\
i \\
1 \\
x \\
x \\
x \\
x\end{array}$ & \begin{tabular}{l|l|}
$x$ & \\
$x$ & \\
$x$ & \\
$x$ & \\
$x$ & \\
$x$ & \\
$x$ & \\
$x$ &
\end{tabular} & $\begin{array}{l}x \\
x \\
x \\
x \\
x \\
x \\
x \\
x\end{array}$ & $\begin{array}{ll}1 & 1 \\
1 & \\
& \\
1 & \\
x & \\
x & \\
x & \\
\end{array}$ & 1 & $\begin{array}{|lll|}x & & \\
& 1 & \\
1 & & \\
& & \\
x & 1 & 1 \\
x & & \\
\end{array}$ & 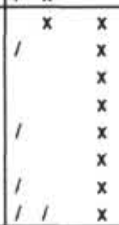 & $\begin{array}{l}C \\
F \\
F \\
F \\
R \\
F \\
F \\
F\end{array}$ & $\begin{array}{lll}F & F \\
A & C \\
A & A \\
A & C & B \\
A & A \\
A & A \\
A & A \\
R & A & A \\
F & F\end{array}$ & $\begin{array}{l}A \\
A \\
A \\
A \\
A \\
A \\
A \\
A \\
A \\
A\end{array}$ & $\begin{array}{l}p \\
\text { VP } \\
\text { VP } \\
\text { VP } \\
\text { VP } \\
\text { VP } \\
P \\
P\end{array}$ \\
\hline $\begin{array}{l}\text { Upper } \\
\text { Miocene }\end{array}$ & $\begin{array}{l}\text { N17 } \\
\text { to }\end{array}$ & $\begin{array}{l}6 H-1,105-110 \\
6 H-2,40-45 \\
6 H-3,105-110 \\
6 H-4,10-15 \\
6 H-5,40-45 \\
6 H-6,40-45 \\
6 H-C C \\
7 H-1,105-110 \\
7 H-2,105-110 \\
7 H-3,105-110 \\
7 H-4,105-110 \\
7 H-5,105-110 \\
7 H-6 \\
7 H-c c \\
8 H-1,105-110 \\
8 H-2,105-110 \\
8 H-3,105-110 \\
8 H-4,105-110 \\
8 H-5,105-110 \\
8 H-6,105-110 \\
8 H-C C \\
9 H-1,105-110 \\
9 H-2,105-110 \\
9 H-3,105-110 \\
9 H-4,105-110 \\
\end{array}$ & $\begin{array}{l}48.95 \\
49.80 \\
51.95 \\
52.50 \\
54.30 \\
55.80 \\
57.50 \\
58.55 \\
60.05 \\
61.55 \\
63.05 \\
64.55 \\
66.05 \\
67.10 \\
68.15 \\
69.65 \\
71.15 \\
72.65 \\
74.15 \\
75.65 \\
76.60 \\
77.65 \\
79.15 \\
80.65 \\
82.15 \\
\end{array}$ & 1 & $\hat{\imath}$ & $\mid \begin{array}{l}\mathbf{x} \\
\mathrm{x} \\
1 \\
1 \\
1\end{array}$ & $\begin{array}{l}x \\
x \\
x \\
x \\
x \\
x \\
y \\
y \\
\prime \\
x \\
x \\
x \\
x \\
x \\
y\end{array}$ & $\begin{array}{l}1 \\
1 \\
1 \\
1 \\
1 \\
x \\
x \\
1 \\
1 \\
\\
x \\
x \\
x \\
x \\
\\
x \\
x \\
x\end{array}$ & & $\begin{array}{l}1 \\
1 \\
1\end{array}$ & $\begin{array}{l}1 \\
x \\
x\end{array}$ & 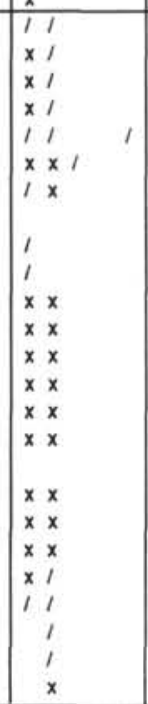 & $\begin{array}{l}x \\
x \\
x \\
x \\
x \\
x \\
x \\
x \\
1 \\
y \\
y \\
x \\
x \\
x \\
x \\
x \\
x \\
i \\
x \\
x \\
x \\
x \\
x \\
1 \\
y \\
x\end{array}$ & $\begin{array}{l}C \\
C \\
F \\
C \\
F \\
F \\
F \\
F \\
C \\
F \\
F \\
C \\
F \\
C \\
F \\
C \\
\text { A } \\
F \\
F \\
C \\
C \\
C \\
A \\
C \\
A \\
\end{array}$ & $\begin{array}{lll}A & A \\
A & A \\
A & C \\
A & C \\
A & C \\
A & A \\
A & A & C \\
C & C \\
A & A \\
A & F \\
A & C \\
A & A & C \\
A & F \\
A & C \\
A & C \\
C & C \\
A & A \\
A & A & A \\
A & C \\
C & C \\
A & C \\
A & C \\
A & C \\
A & A & A \\
A & A & R \\
A & A & F \\
A & A & F\end{array}$ & $\begin{array}{l}\text { C } \\
A \\
C \\
C \\
C \\
A \\
C \\
A \\
\text { F } \\
\text { F } \\
\text { A } \\
A \\
A \\
A \\
A \\
A \\
A \\
A \\
\text { A } \\
\text { A } \\
\text { A } \\
\text { A } \\
\text { C } \\
\text { A } \\
\text { F } \\
\text { F } \\
\text { C }\end{array}$ & $\begin{array}{l}\text { VP } \\
\text { VP } \\
\text { VP } \\
\text { VP } \\
\text { VP } \\
p \\
\text { VP } \\
\text { VP } \\
\text { VP } \\
\text { VP } \\
P \\
P \\
P \\
p \\
p \\
\text { VP } \\
\text { VP } \\
P \\
P \\
P \\
\text { VP } \\
\text { VP } \\
\text { VP } \\
\text { VP } \\
\text { VP }\end{array}$ \\
\hline
\end{tabular}




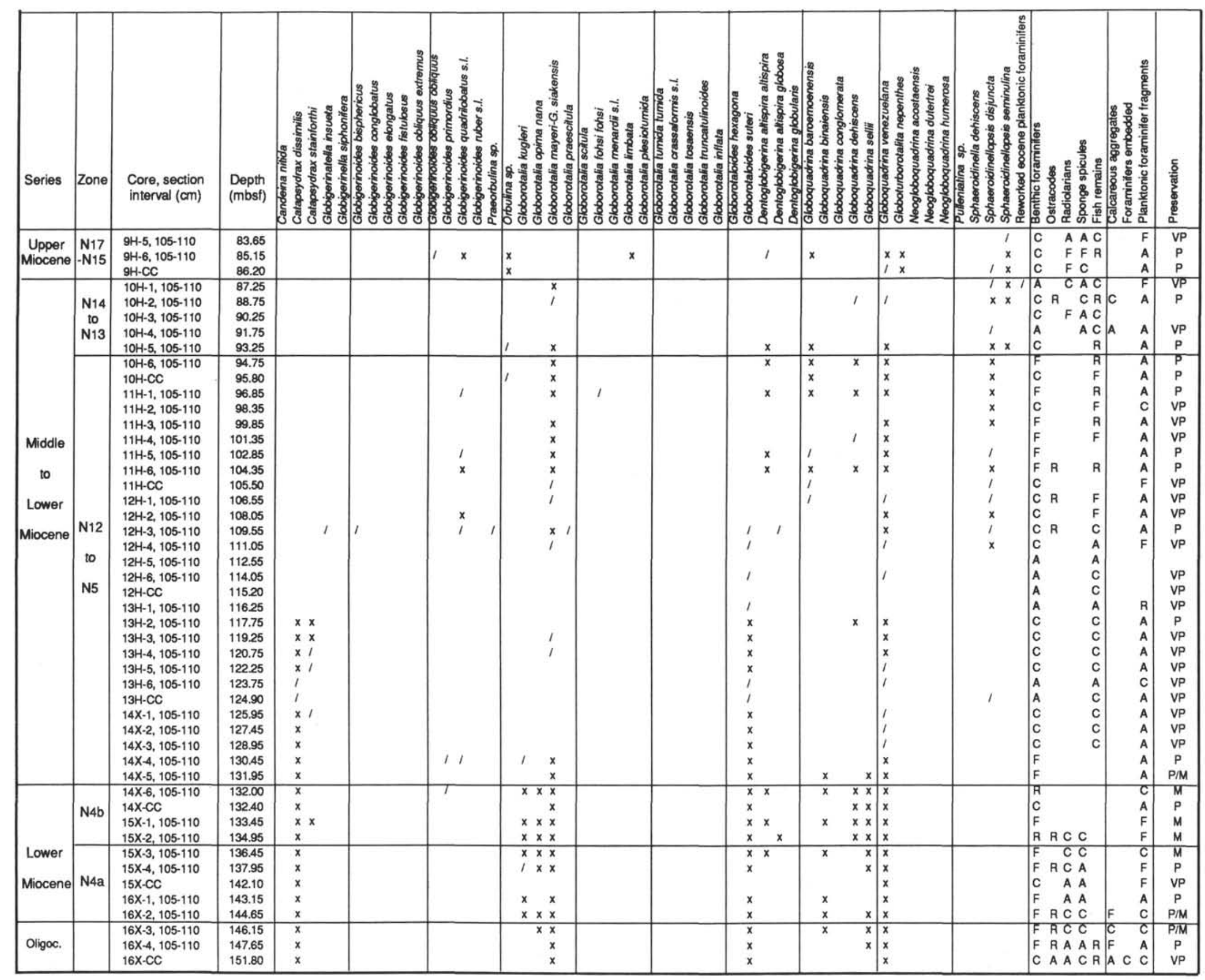


Table 9. Distribution of Neogene planktonic foraminifers in sediments of Hole 711A.

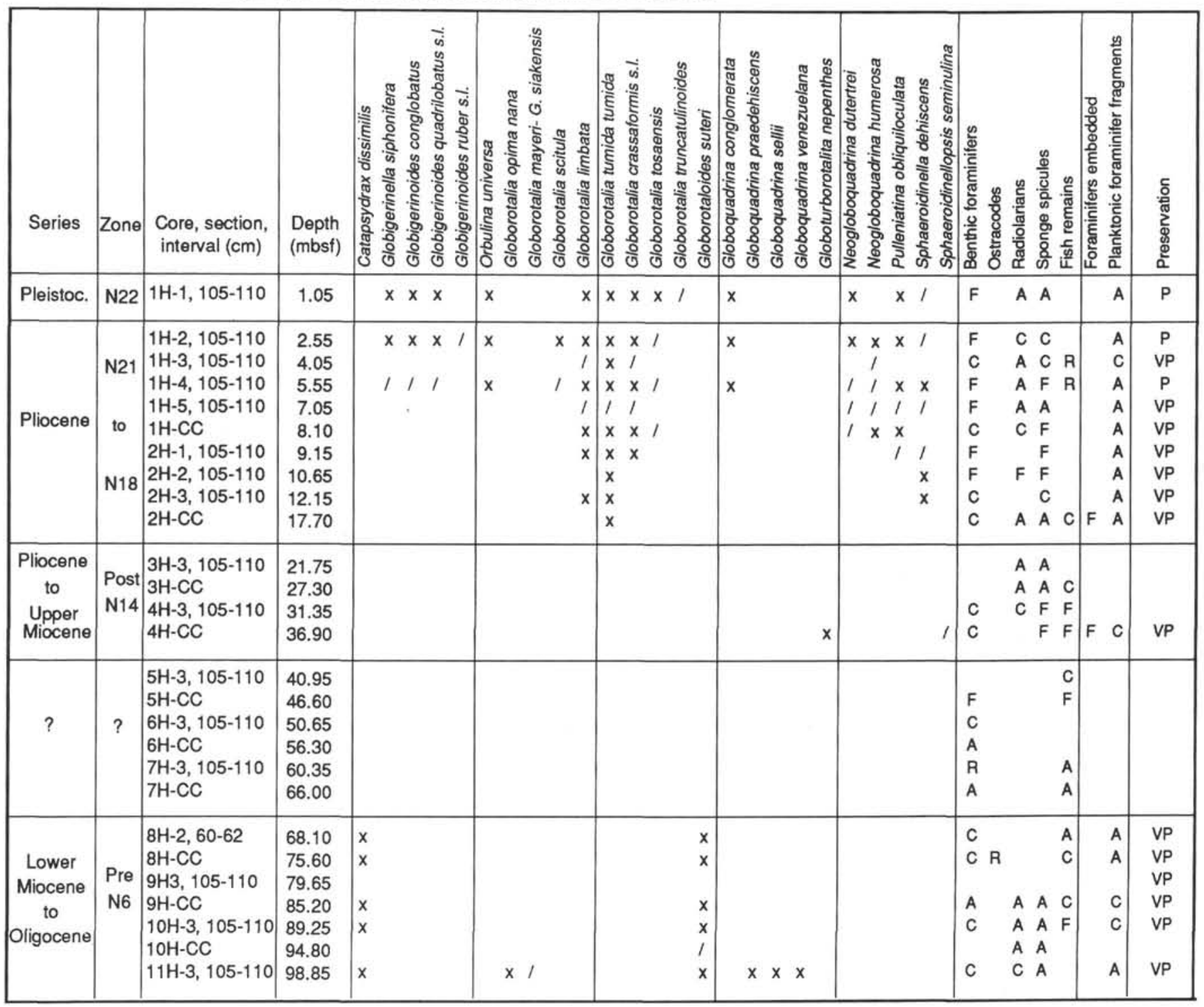


Table 10. Distribution of Neogene planktonic foraminifers in sediments of Hole 712A.

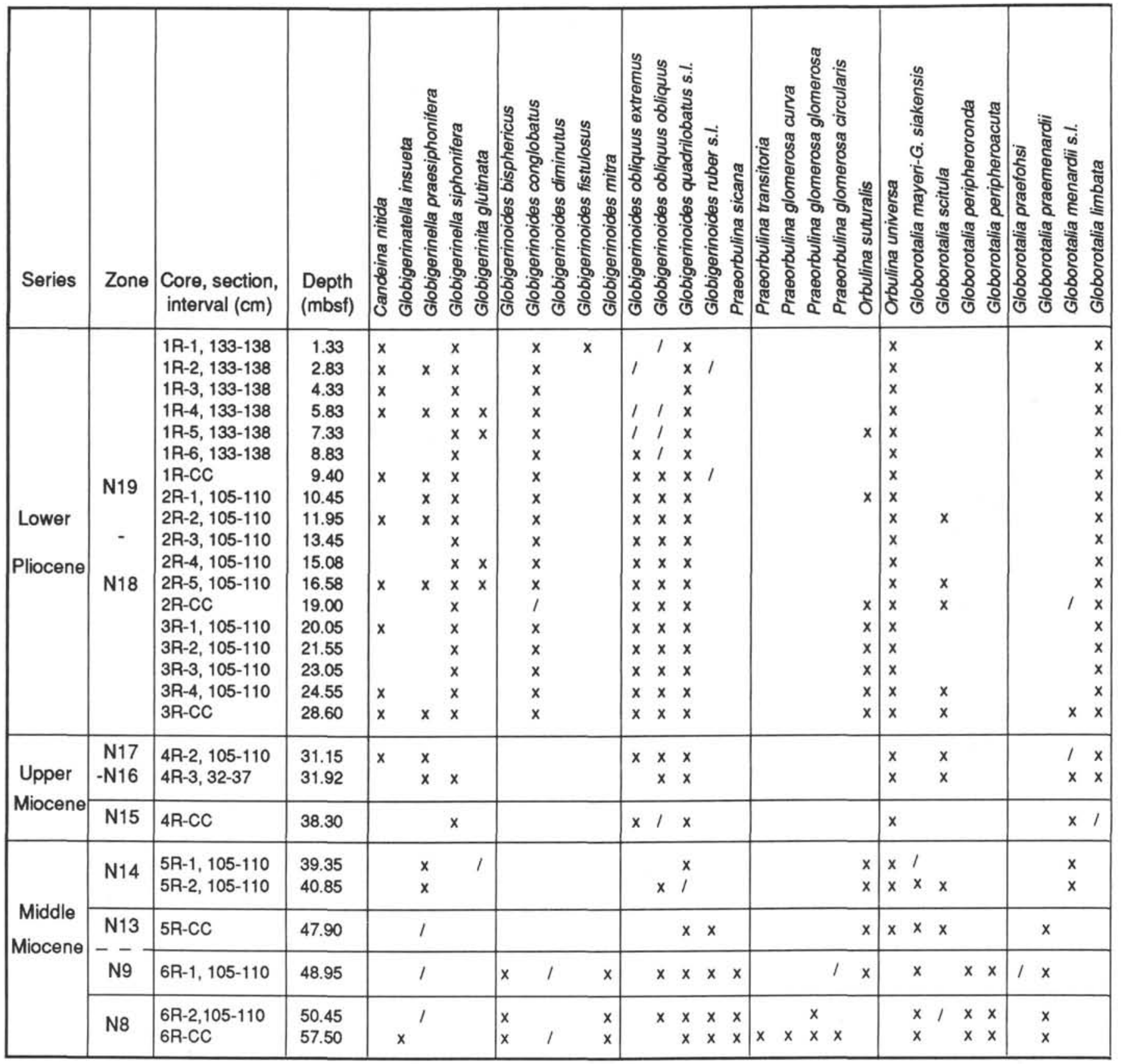


Table 10 (Continued).

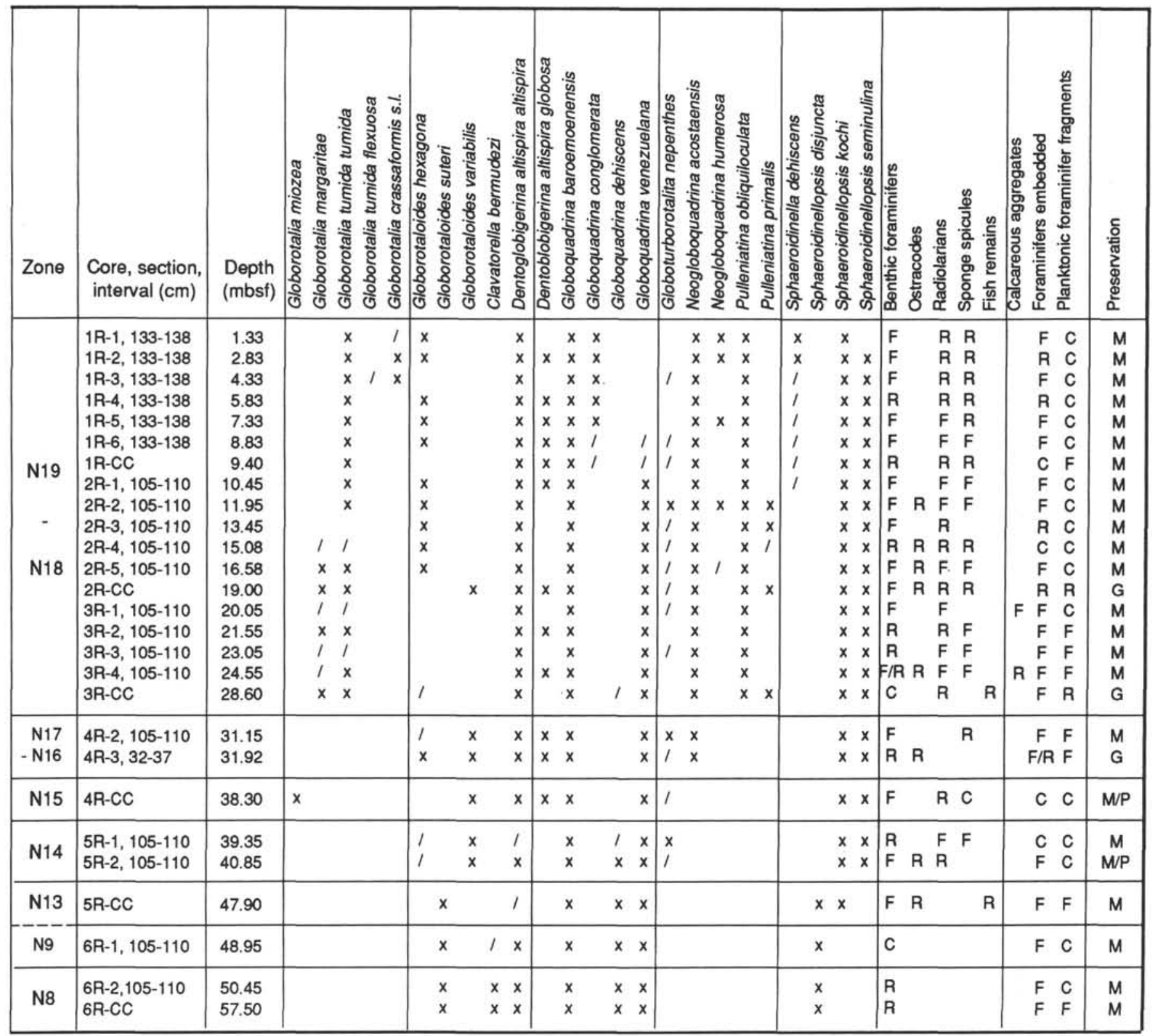


Table 11. Distribution of Neogene planktonic foraminifers in sediments of Hole 713A.

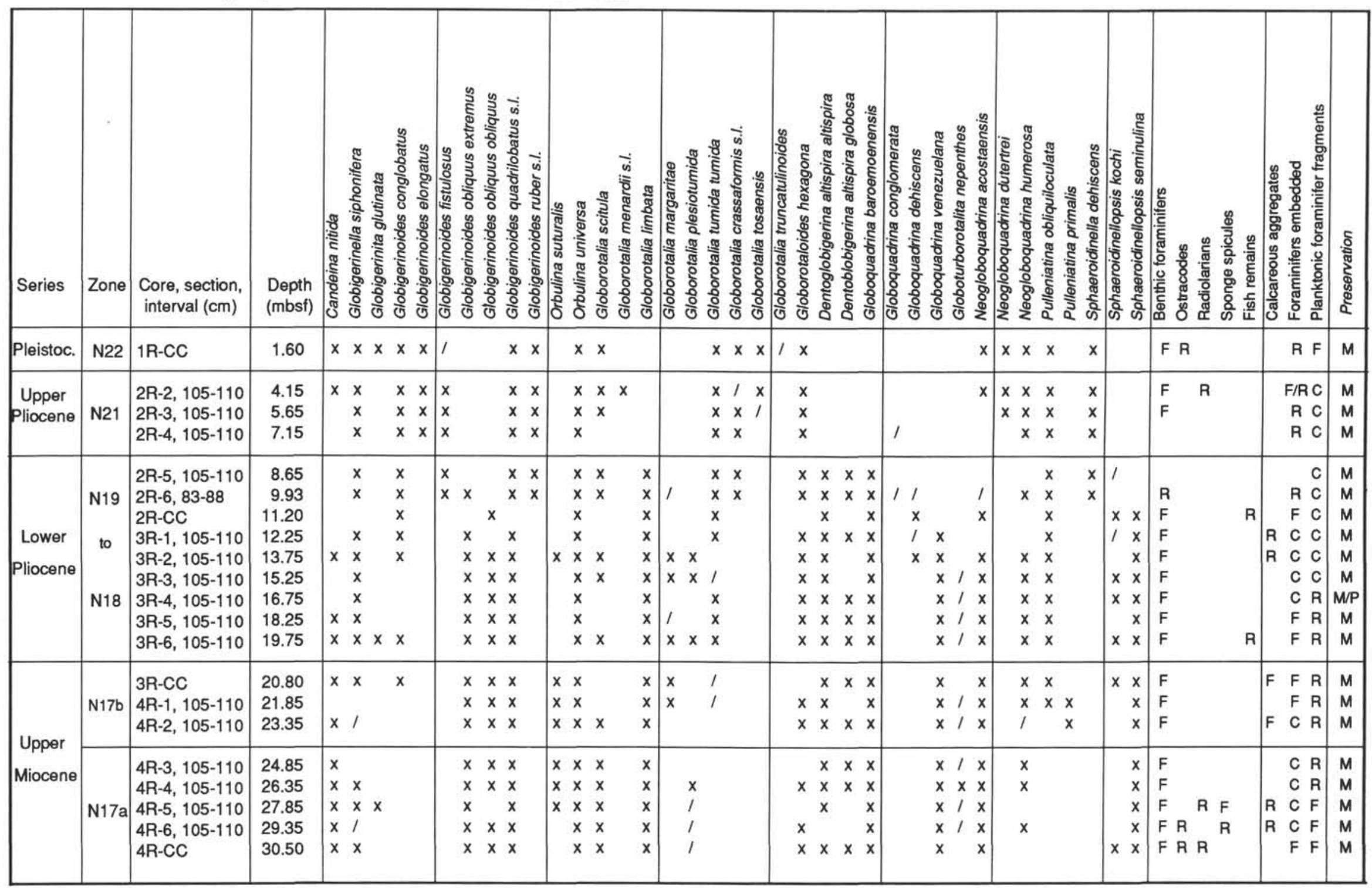


Table 12. Distribution of Neogene planktonic foraminifers in sediments of Hole 714A.

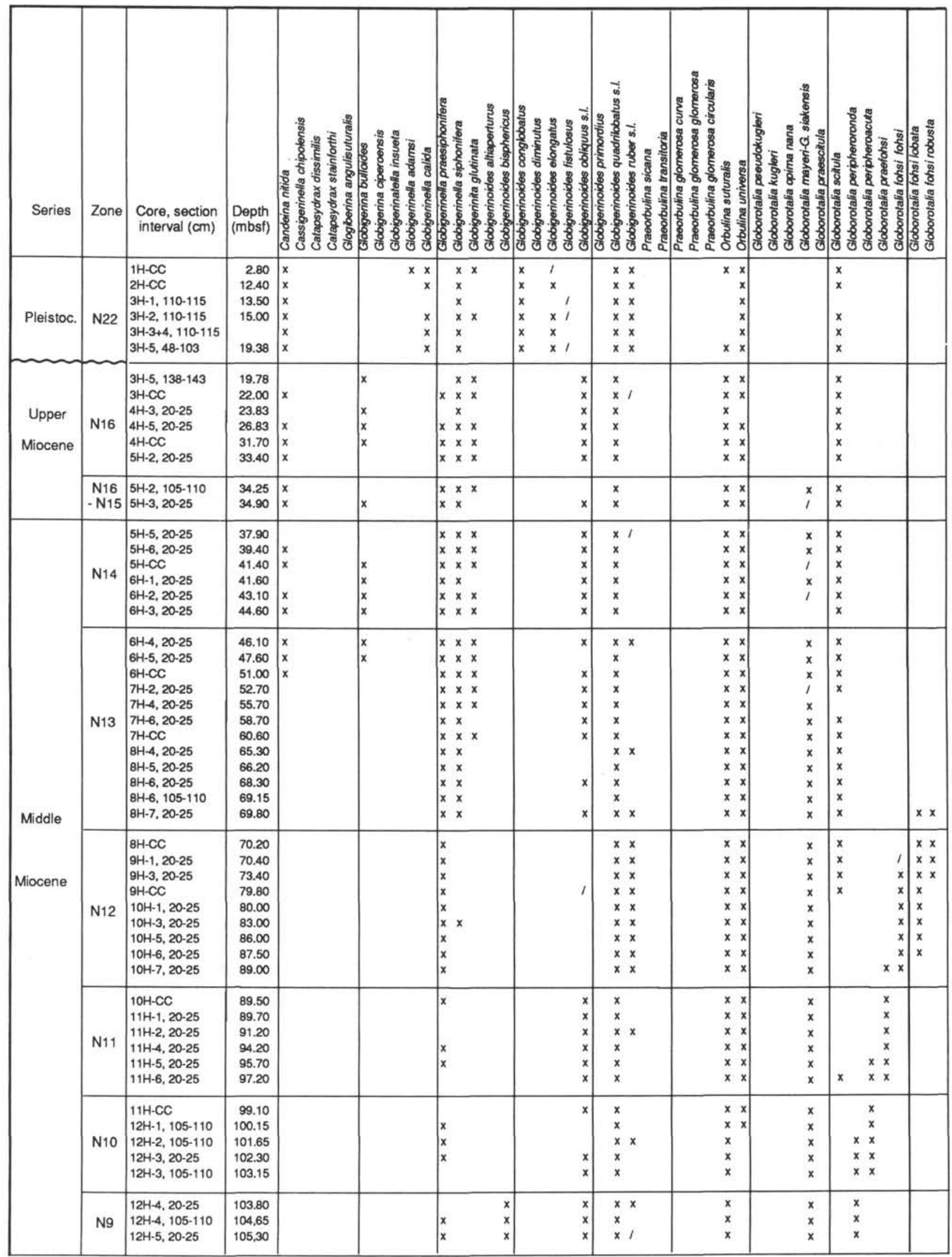


Table 12 (Continued).

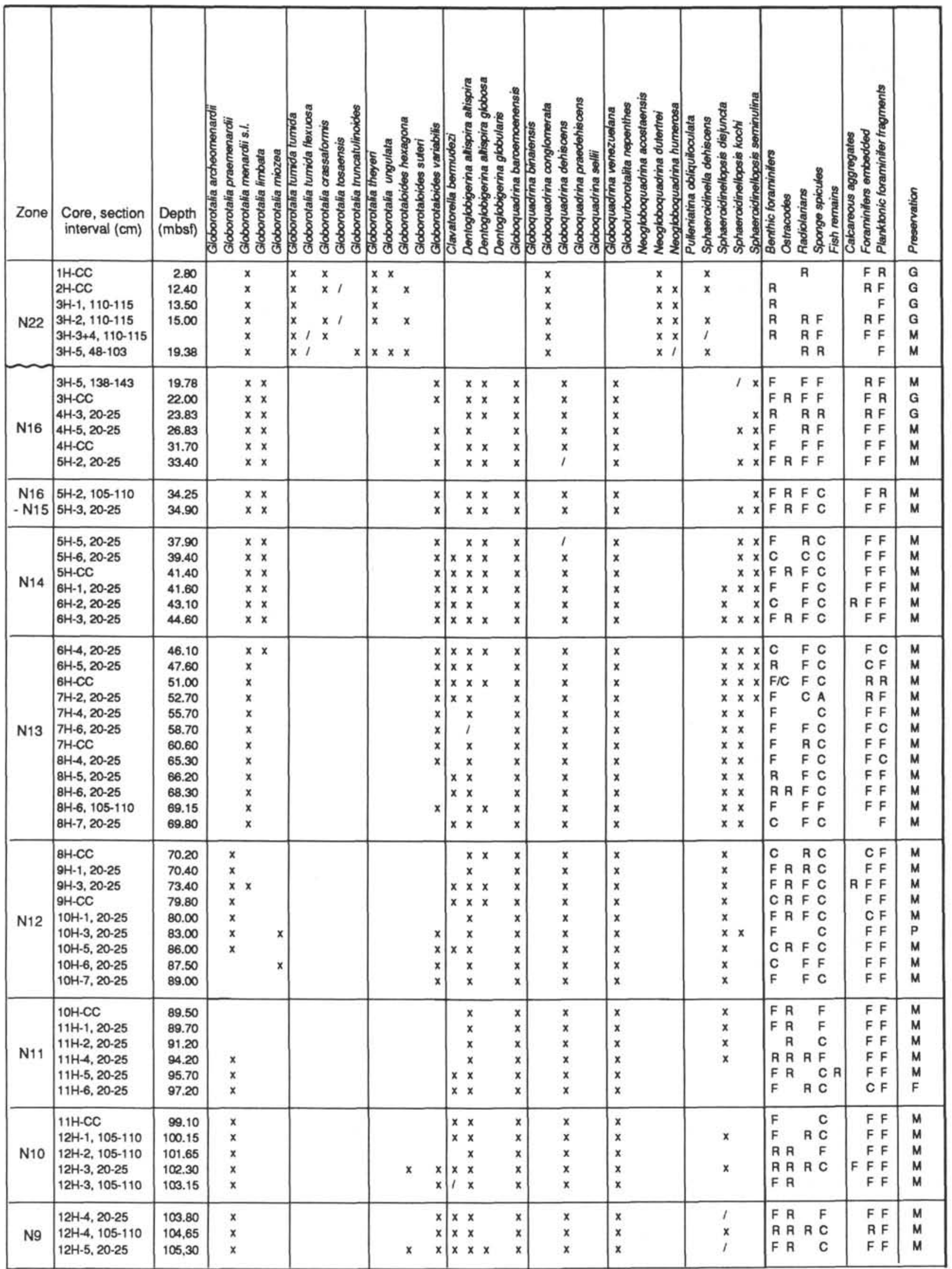


Table 12 (Continued).

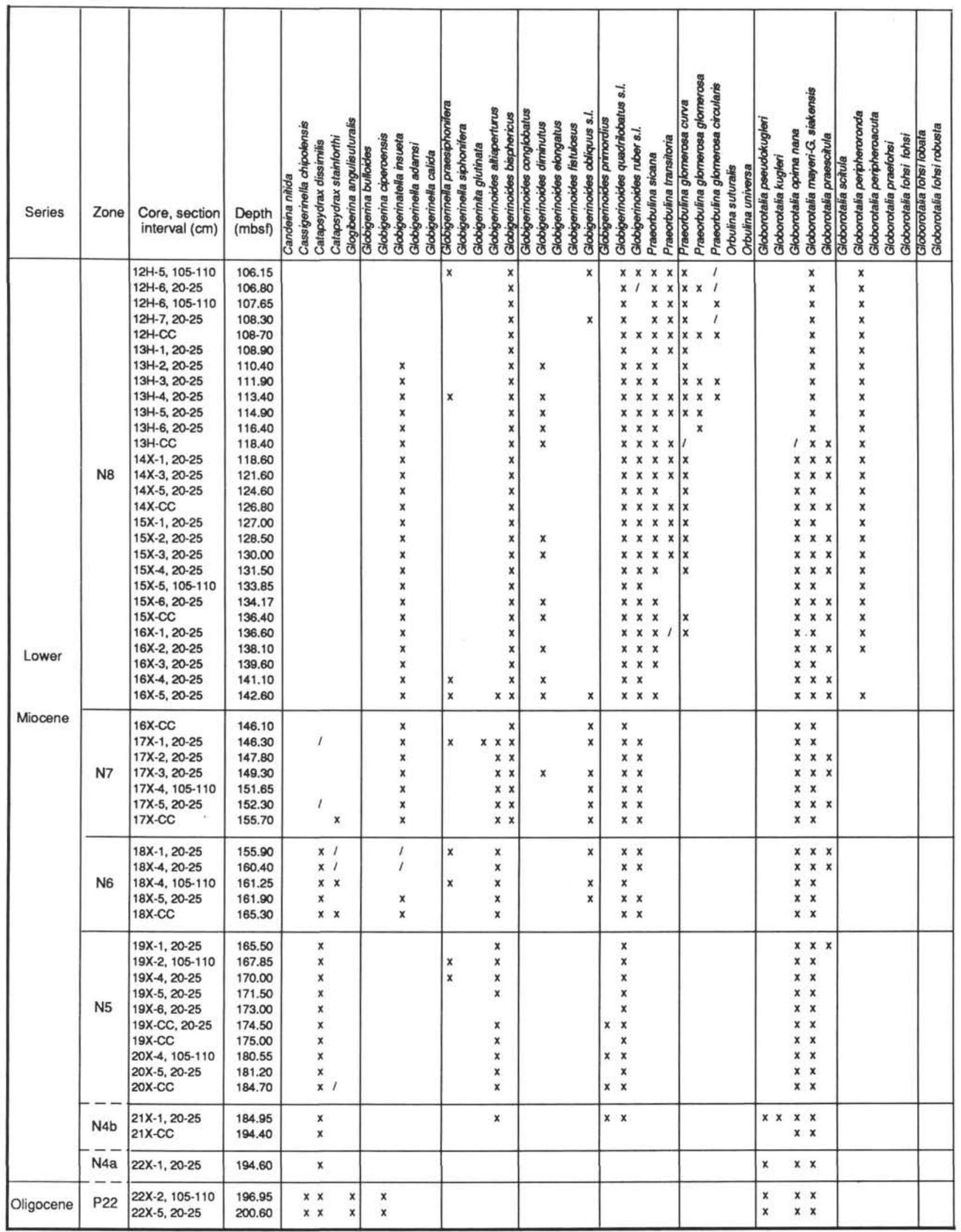


Table 12 (Continued).

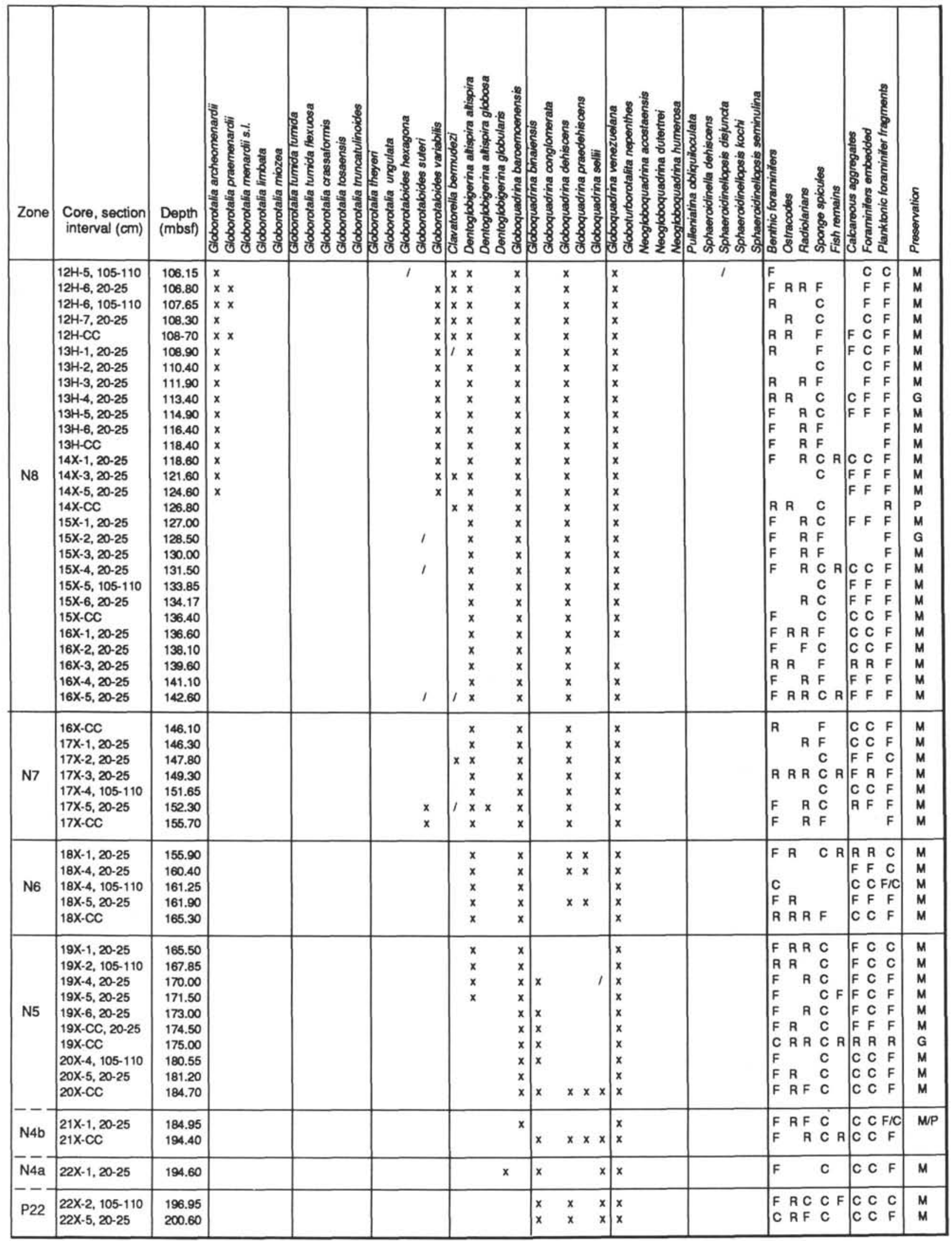


Table 13. Distribution of Neogene planktonic foraminifers in sediments of Hole 715A.

\begin{tabular}{|c|c|c|c|c|c|c|c|c|c|c|c|}
\hline Series & Zone & $\begin{array}{l}\text { Core, section } \\
\text { interval (cm) }\end{array}$ & $\begin{array}{l}\text { Depth } \\
\text { (mbsf) }\end{array}$ & 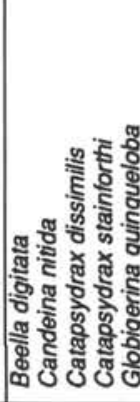 & 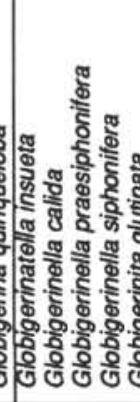 & 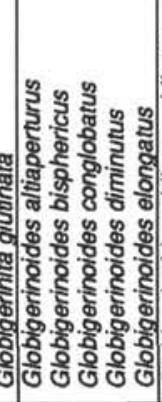 & 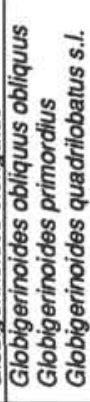 & 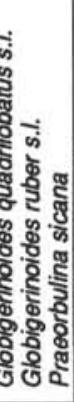 & 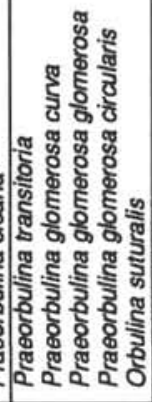 & 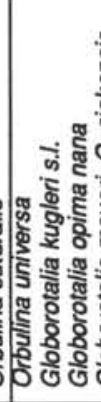 & 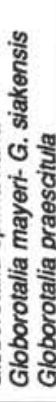 \\
\hline Pleistoc. & N22 & $\begin{array}{l}1 \mathrm{R}-\mathrm{CC} \\
\text { 2R-CC } \\
3 \mathrm{R}-\mathrm{CC} \\
4 \mathrm{R}-\mathrm{CC} \\
5 \mathrm{R}-\mathrm{CC}\end{array}$ & $\begin{array}{r}8.10 \\
17.80 \\
27.50 \\
37.10 \\
46.80\end{array}$ & $\begin{array}{lll}x & x & x \\
l & x & \\
& x & \\
& x & \\
& x & \end{array}$ & $\begin{array}{lll}x & x & x \\
x & x & x \\
x & x & \\
& x & x \\
x & x\end{array}$ & $\begin{array}{cc}x & x \\
x & \\
x & x \\
x & x \\
x & \end{array}$ & & $\begin{array}{ll}x & x \\
x & x \\
x & x \\
x & x \\
x & x\end{array}$ & & $\begin{array}{l}x \\
x \\
x \\
x \\
x\end{array}$ & \\
\hline \multirow[b]{4}{*}{$\begin{array}{l}\text { Middle } \\
\text { Miocene }\end{array}$} & N12 & $\begin{array}{l}\text { 6R-1, } 105-110 \\
6 \mathrm{R}-2,105-110 \\
6 \mathrm{R}-3,105-110\end{array}$ & $\begin{array}{l}47.85 \\
49.35 \\
50.85\end{array}$ & & $\begin{array}{l}x \\
x \\
\end{array}$ & & $\begin{array}{ll}x & x \\
x & x \\
x & x \\
\end{array}$ & $\begin{array}{ll}x & x \\
x & x \\
x & x \\
\end{array}$ & $\begin{array}{l}x \\
x \\
x\end{array}$ & $\begin{array}{l}x \\
x \\
x\end{array}$ & $\begin{array}{l}x \\
x \\
x\end{array}$ \\
\hline & $\begin{array}{l}\text { N11. } \\
\text { N10 }\end{array}$ & $\begin{array}{l}6 R-4,105-110 \\
6 R-5,105-110\end{array}$ & $\begin{array}{l}52.35 \\
53.85\end{array}$ & & $\begin{array}{l}x \\
x\end{array}$ & & $\begin{array}{l}x \\
x\end{array}$ & $\begin{array}{l}x \times \\
x\end{array}$ & $\begin{array}{l}x \\
x\end{array}$ & $\begin{array}{l}x \\
x\end{array}$ & $\begin{array}{l}x \\
x\end{array}$ \\
\hline & N9 & $\begin{array}{l}\text { 6R-6, } 105-110 \\
6 \mathrm{R}-\mathrm{CC}\end{array}$ & $\begin{array}{l}55.35 \\
56.50\end{array}$ & & 1 & $x$ & $x$ & $\operatorname{lr}_{x} x$ & $\begin{array}{ll} & x \\
x & x\end{array}$ & $x$ & $\begin{array}{l}x \\
x \times\end{array}$ \\
\hline & N8 & $\begin{array}{l}\text { 7R-1, 105-110 } \\
\text { 7R-2, 105-110 } \\
\text { 7R-3, 105-110 } \\
\text { 7R-4, 105-110 } \\
\text { 7R-5, 105-110 } \\
\text { 7R-6, 105-110 } \\
\text { 7R-CC } \\
\text { 8R-1, 105-110 } \\
\text { 8R-2, 105-110 } \\
\text { 8R-3, 105-110 } \\
\text { 8R-4, 105-110 } \\
\text { 8R-5, 105-110 } \\
\text { 8R-6, 105-110 } \\
\text { 8R-CC }\end{array}$ & $\begin{array}{l}57.55 \\
59.05 \\
60.55 \\
62.05 \\
63.55 \\
65.05 \\
66.10 \\
67.15 \\
68.65 \\
70.15 \\
71.65 \\
73.15 \\
74.65 \\
75.70\end{array}$ & & $\begin{array}{ll}x & \\
x & \\
x & \\
x & \\
x & x \\
x & \\
x & \\
x & \\
x & \\
x & \\
x & \\
x & \\
x & \end{array}$ & $\begin{array}{ll}\mathrm{x} & \\
\mathrm{x} & \\
\mathrm{x} & \\
\mathrm{x} & \\
\mathrm{x} & \mathrm{x} \\
\mathrm{x} & \mathrm{x} \\
\mathrm{x} & \mathrm{x} \\
\mathrm{x} & \mathrm{x} \\
\mathrm{x} & \mathrm{x} \\
\mathrm{x} & \mathrm{x} \\
\mathrm{x} & \mathrm{x} \\
\mathrm{x} & \mathrm{x} \\
\mathrm{x} & \mathrm{x} \\
\mathrm{x} & \end{array}$ & & $\begin{array}{lll}x & x & x \\
x & x & x \\
x & x & x \\
x & x & x \\
x & x & x \\
x & x & x \\
x & x & x \\
x & 1 & x \\
x & x & x \\
x & x & x \\
x & x & x \\
x & x & x \\
x & I & x \\
x & x & x\end{array}$ & 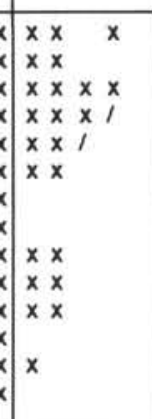 & $\begin{array}{l} \\
x \\
x \\
x\end{array}$ & $\begin{array}{l}x \\
x \\
x \\
x \\
x \\
x \\
x \\
x \\
x \\
x \\
x \\
x \\
x \\
x \\
x \\
x \\
x\end{array}$ \\
\hline \multirow{4}{*}{$\begin{array}{l}\text { Lower } \\
\text { Miocene }\end{array}$} & N7 & \begin{tabular}{|l|} 
9R-1, $105-110$ \\
9R-2, 105-110 \\
9R-3, 105-110 \\
9R-4, 105-110 \\
9R-5, 105-110
\end{tabular} & $\begin{array}{l}76.75 \\
78.25 \\
79.75 \\
81.25 \\
82.75\end{array}$ & & $\begin{array}{ll}x & \\
x & \\
x & \\
x & \\
x & I\end{array}$ & $\begin{array}{ll} & x \\
x & \\
x & \\
x & 1 \\
x & \end{array}$ & $\begin{array}{l}x \\
x \\
x \\
x \\
x\end{array}$ & $\begin{array}{ll}x & x \\
x & x \\
x & x \\
x & x \\
x & x\end{array}$ & & $\begin{array}{l}x \\
x \\
x \\
x\end{array}$ & $\begin{array}{l}x \quad x \\
x \\
x \\
x \\
x\end{array}$ \\
\hline & N6 & $\begin{array}{l}\text { 9R-6, 105-110 } \\
\text { 9R-CC }\end{array}$ & $\begin{array}{l}84.25 \\
85.30\end{array}$ & $\begin{array}{l}x \\
x\end{array}$ & $\begin{array}{l}x \\
x\end{array}$ & 1 & $x$ & $\begin{array}{l}x \times \\
x \times\end{array}$ & & & $\begin{array}{l}x \\
x\end{array}$ \\
\hline & N5 & $\begin{array}{l}\text { 10R-2, 105-106 } \\
\text { 10R-3, 105-110 } \\
\text { 10R-4, 105-110 } \\
\text { 10R-5, 105-110 } \\
\text { 10R-6, 105-110 } \\
\text { 10R-CC } \\
\text { 11R-1, 105-110 } \\
\text { 11R-2, 105-110 } \\
\text { 11R-3, 105-110 } \\
11 \mathrm{R}-4,105-110\end{array}$ & $\begin{array}{r}87.85 \\
89.35 \\
90.85 \\
92.35 \\
93.85 \\
94.90 \\
95.95 \\
97.45 \\
98.95 \\
100.45 \\
\end{array}$ & 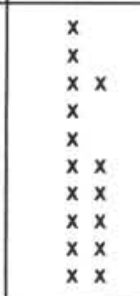 & 1 & $\begin{array}{l}x \\
\prime \\
x \\
x \\
x \\
x \\
x\end{array}$ & $\begin{array}{lll} & x & x \\
x & & x \\
x & x & x \\
x & & x \\
1 & & x \\
x & x & x \\
x & x & x \\
x & x & x \\
x & x & x \\
& 1 & x\end{array}$ & $\begin{array}{ll}x & \prime \\
x & 1 \\
x & \\
x & x \\
x & 1 \\
x & \prime \\
x & x \\
x & x \\
x & x \\
x & \end{array}$ & & $\begin{array}{l} \\
x \\
x \\
x \\
x \\
x \\
x \\
x \\
x\end{array}$ & $\begin{array}{l}x \\
x \\
x \\
x \\
x \\
x \\
x \\
x \\
x \\
x\end{array}$ \\
\hline & N4b & $\begin{array}{l}\text { 11R-5, 105-110 } \\
\text { 11R-6, 105-110 } \\
\text { 11R-CC }\end{array}$ & $\begin{array}{l}101.95 \\
103.45 \\
104.60\end{array}$ & $\begin{array}{ll}x & x \\
x & x \\
x & x\end{array}$ & & $\begin{array}{l}x \\
x \\
x\end{array}$ & $\begin{array}{ll}1 & x \\
x & x \\
x & x\end{array}$ & $\begin{array}{l}x \\
x \\
x\end{array}$ & & $\begin{array}{ll}1 & x \\
x & x \\
x & x\end{array}$ & $\begin{array}{l}x \\
x\end{array}$ \\
\hline
\end{tabular}


Table 13 (Continued).

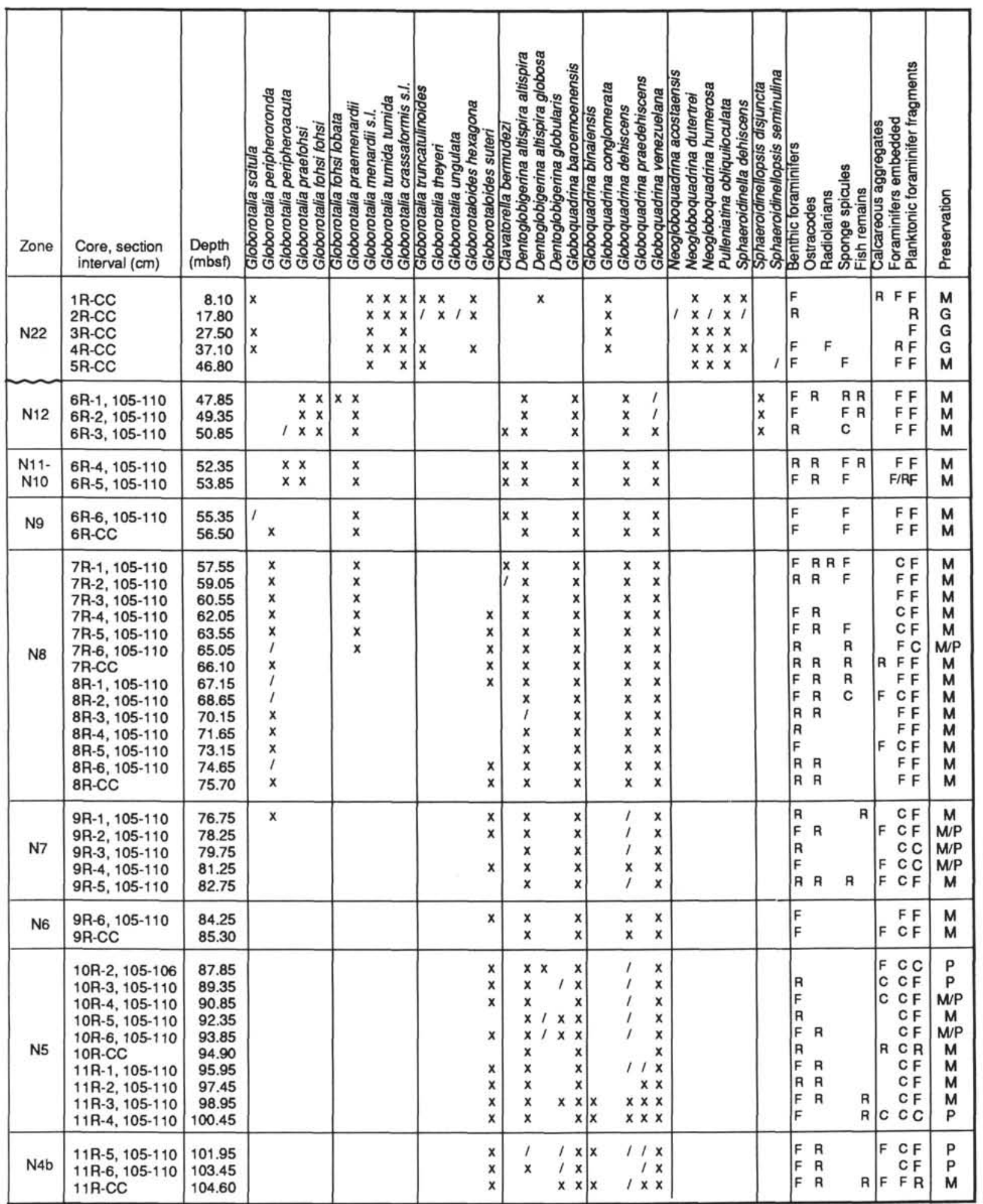


Table 14. Distribution of Neogene planktonic foraminifers in sediments of Hole $716 \mathrm{~B}$.

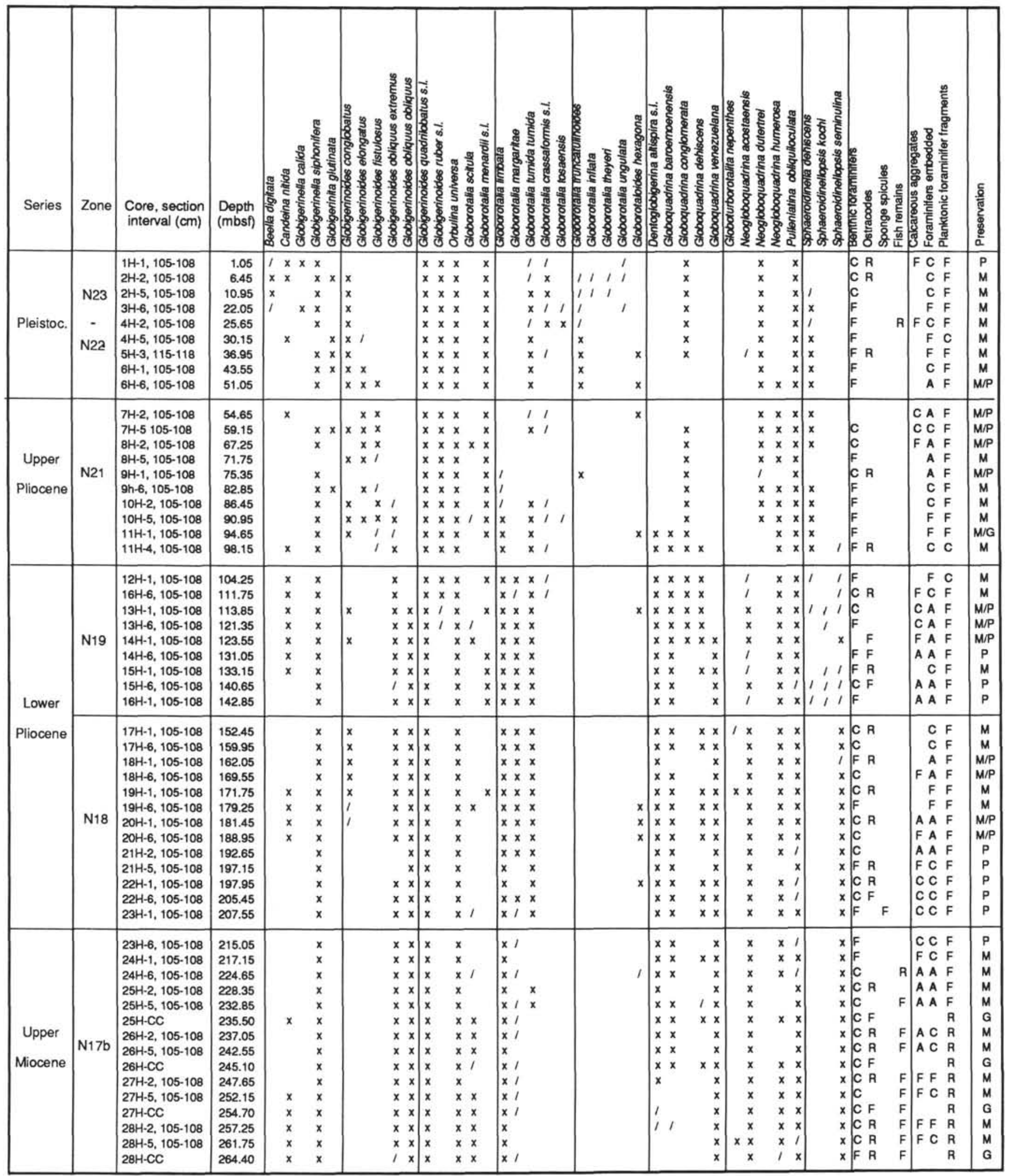


Table 15. Assignment of Leg 115 samples to the Neogene planktonic foraminifer zonation. Uppermost and lowermost samples assigned to each zone are indicated. Numbers in parentheses are sub-bottom depth (mbsf) of the top of these samples.

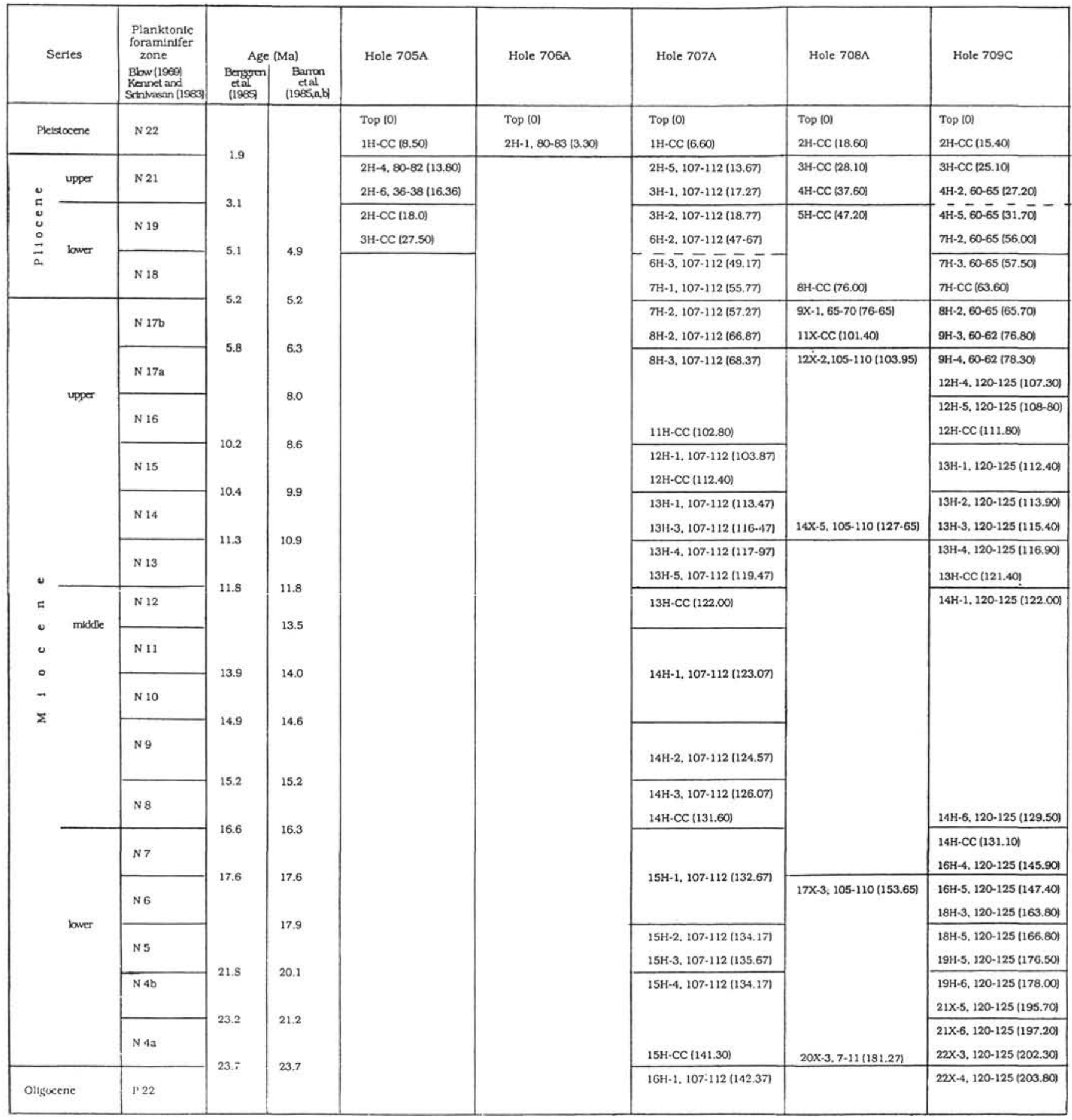


Table 15 (Continued).

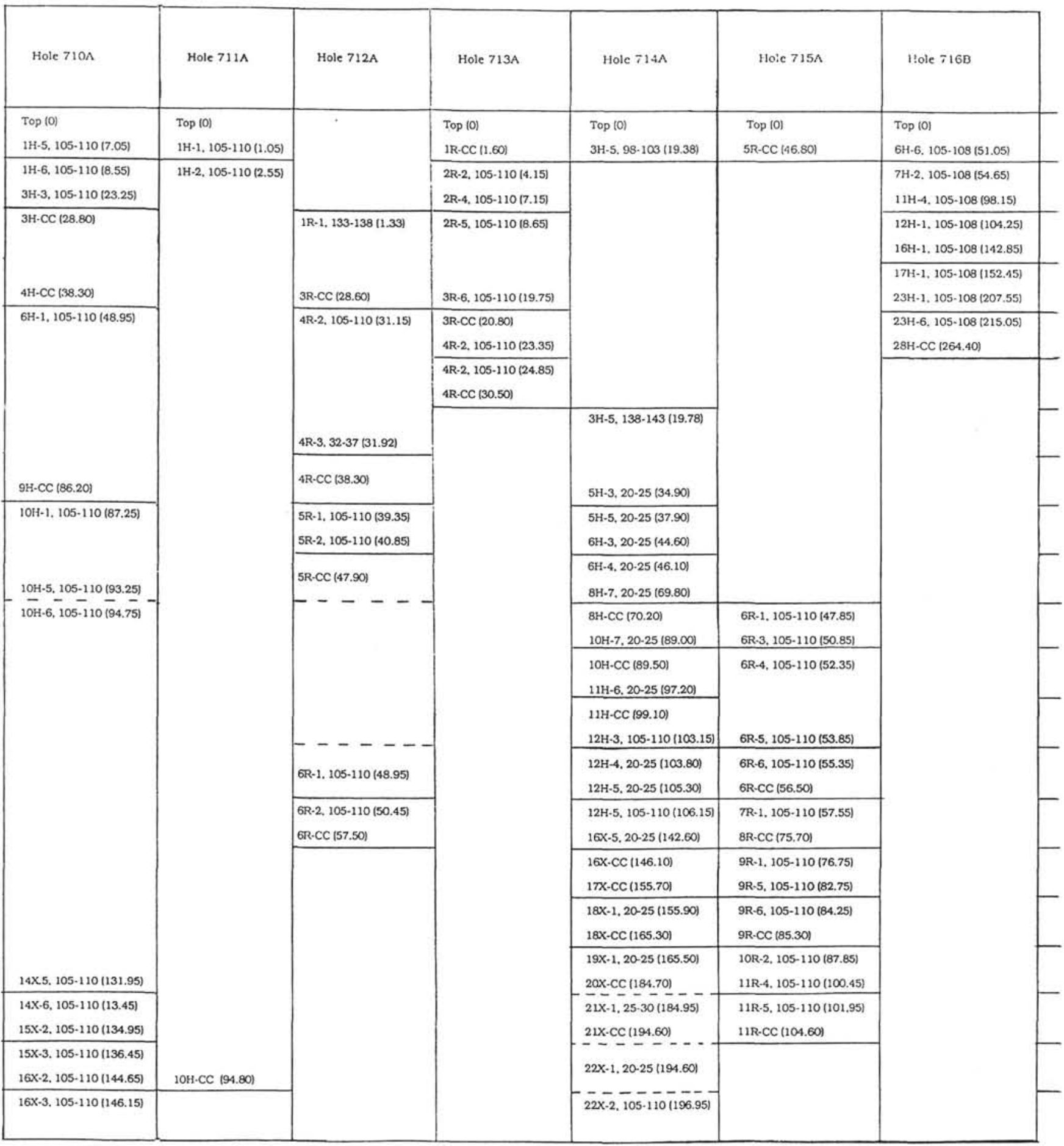




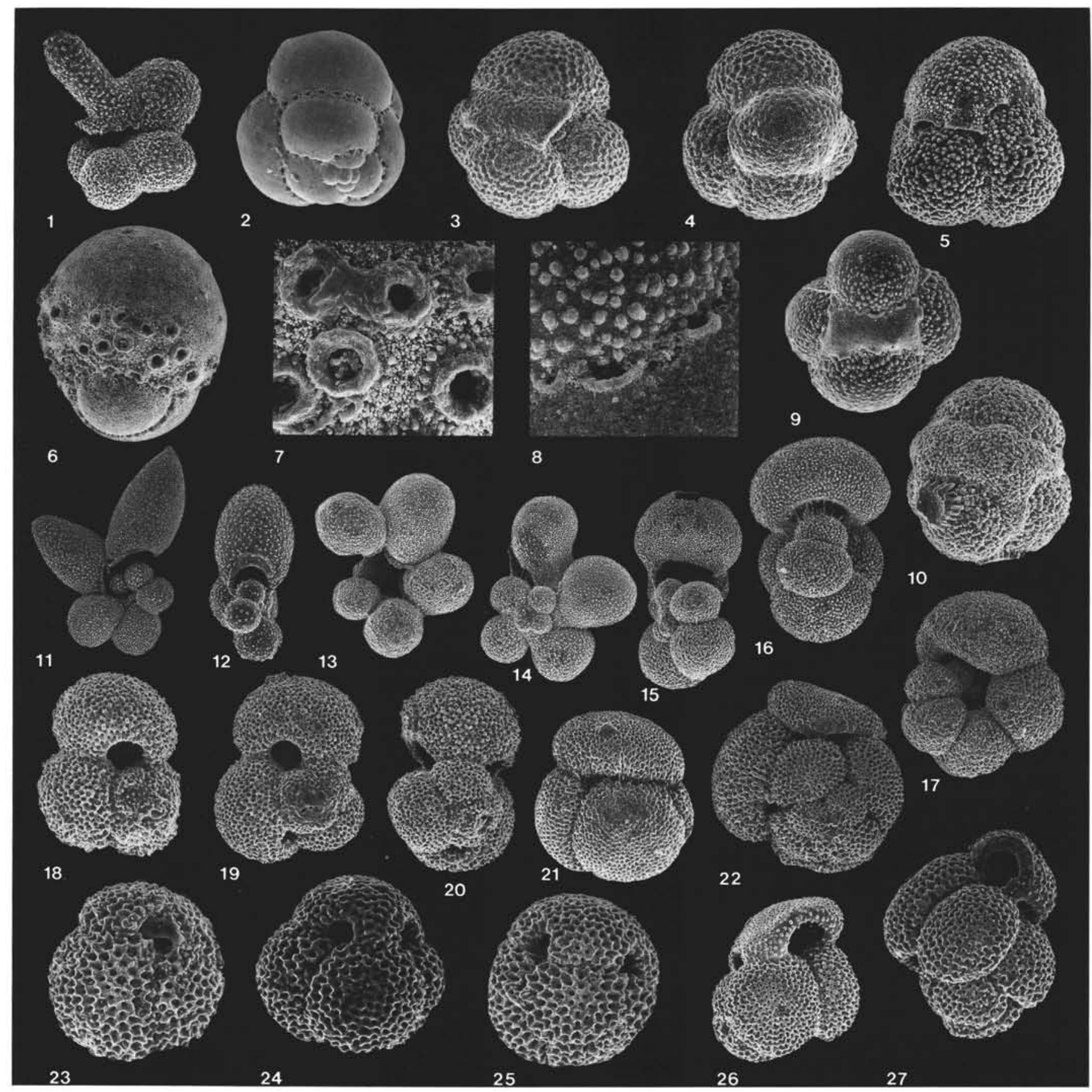

Plate 1. 1. Beella digitata; Sample 115-714A-1H-CC, $\times 60$. 2. Candeina nitida; Sample $115-715 \mathrm{~A}-4 \mathrm{R}-\mathrm{CC}, \times 52$. 3, 4. Catapsydrax dissimilis; (3) Sample 115-709C-17H-CC, $\times 67$; (4) Sample 115-707A-16H-1, 107-112 cm, $\times 67 . \quad 5,10$. Catapsydrax stainforthi; Sample 115-707A-15H-1, 107$112 \mathrm{~cm} ;(5) \times 97 ;(10) \times 75$. 6, 7. Globigerinatella insueta; Sample $115-709 \mathrm{C}-16 \mathrm{H}-5,120-125 \mathrm{~cm} ;(6) \times 75 ;(7) \times 224$. 8, 9. Globigerinita glutinata; Sample 115-707A-5H-CC; $(8) \times 373 ;(9) \times 97$. 11, 12. Globigerinella adamsi; Sample $115-706 \mathrm{~A}-1 \mathrm{H}-1,80-83 \mathrm{~cm} ;(11) \times 37 ;(12) \times 45$. 1315. Globigerinella calida; Sample 115-715A-1R-CC; $(13,15) \times 37 ;(14) \times 45 . \quad 16,17$. Globigerinella siphonifera; $(16)$ Sample $115-706 \mathrm{~A}-1 \mathrm{H}-1,80-83$ $\mathrm{cm}, \times 45$; (17) Sample 115-706A-1H-CC, $\times 45 . \quad 18-20$. Globigerinoides altiaperturus; Sample 115-707A-15H-3, 107-112 cm; (18) $\times 67 ;(19,20)$ $\times 60$. 21, 22. Globigerinoides conglobatus; (21) Sample $115-706 \mathrm{~A}-1 \mathrm{H}-\mathrm{CC}, \times 45$; (22) Sample $115-706 \mathrm{~A}-1 \mathrm{H}-2,73-76 \mathrm{~cm}, \times 45$. 23-25. Globigerinoides diminutus; Sample 115-707A-14H-CC, $\times 97$. 26, 27. Globigerinoides elongatus; (26) Sample 115-707A-3H-1, 107-112 cm, $\times 60 ;(27)$ Sample 115-705A-2H-6, 36-38 cm, $\times 75$. 


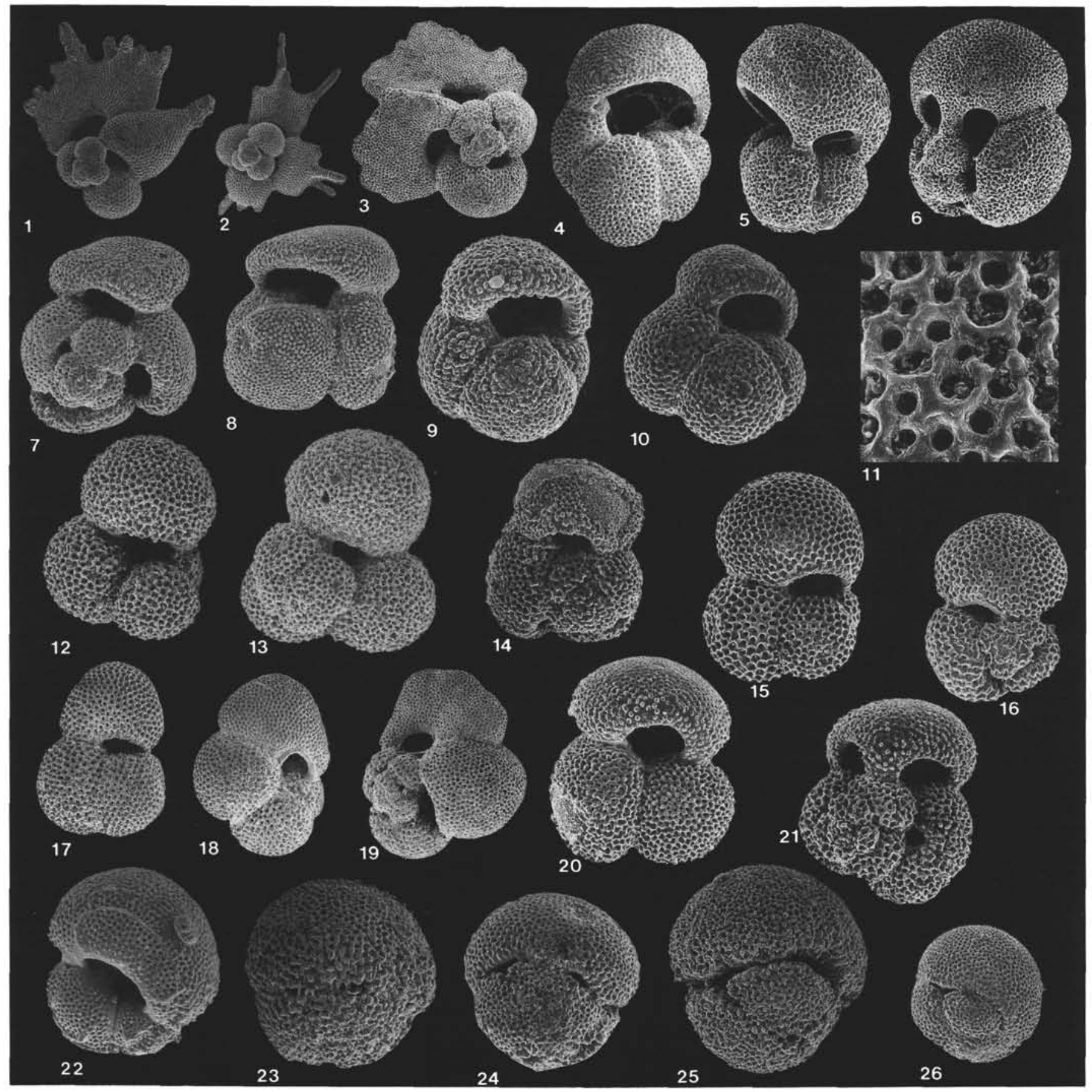

Plate 2. 1-3. Globigerinoides fistulosus; (1) Sample 115-705A-2H-6, 36-38 cm, $\times 27$; (2) Sample 115-707A-3H-1, 107-112 cm, $\times 22$; (3) Sample 115-707A-3H-2, 107-112 cm, $\times 45$. 4-6, 11. Globigerinoides mitra; Sample 115-714A-4H-3, 20-25 cm; (4) $\times 56$; (5) $\times 52 ;(6) \times 45 ;(11) \times 261$. 7, 8. Globigerinoides obliquus extremus; Sample 115-707A-9H-6, 107-112 cm, $\times 52$. 9, 10, 14. Globigerinoides obliquus obliquus; (9, 14) Sample 115-707A-12H-1, 107-112 cm, $\times 67$; (10) Sample 115-716B-28H-CC, $\times 67,12,13$. Globigerinoides primordius; Sample 115-707A-15H-5, 107-112 $\mathrm{cm}, \times 75$. 15, 16. Globigerinoides quadrilobatus trilobus; (15) Sample 115-714A-6H-CC, $\times 67 ;(16)$ Sample 115-709C-7H-CC, $\times 52$. 17-19. Globigerinoides quadrilobatus sacculifer; (17) Sample 115-707A-3H-1, 107-112 cm, $\times 45$; (18) Sample 115-709C-7H-CC, $\times 45$; $(19)$ Sample 115-707A$5 \mathrm{H}-2,107-112 \mathrm{~cm}, \times 37$. 20, 21. Globigerinoides ruber; Sample $115-707 \mathrm{~A}-3 \mathrm{H}-1,107-112 \mathrm{~cm} ;(20) \times 67 ;(21) \times 60$. 22-24. Praeorbulina sicana; $(22,23)$ Sample 115-714A-16X-4, 20-25 cm; (22) $\times 60$; (23) $\times 52$; (24) Sample 115-714A-14X-1, 140-145 cm, $\times 60$. 25, 26. Praeorbulina glomerosa curva; (25) Sample 115-707A-14H-3, 107-112 cm, $\times 52$; (26) Sample 115-714A-13H-5, 140-145 cm, $\times 60$. 


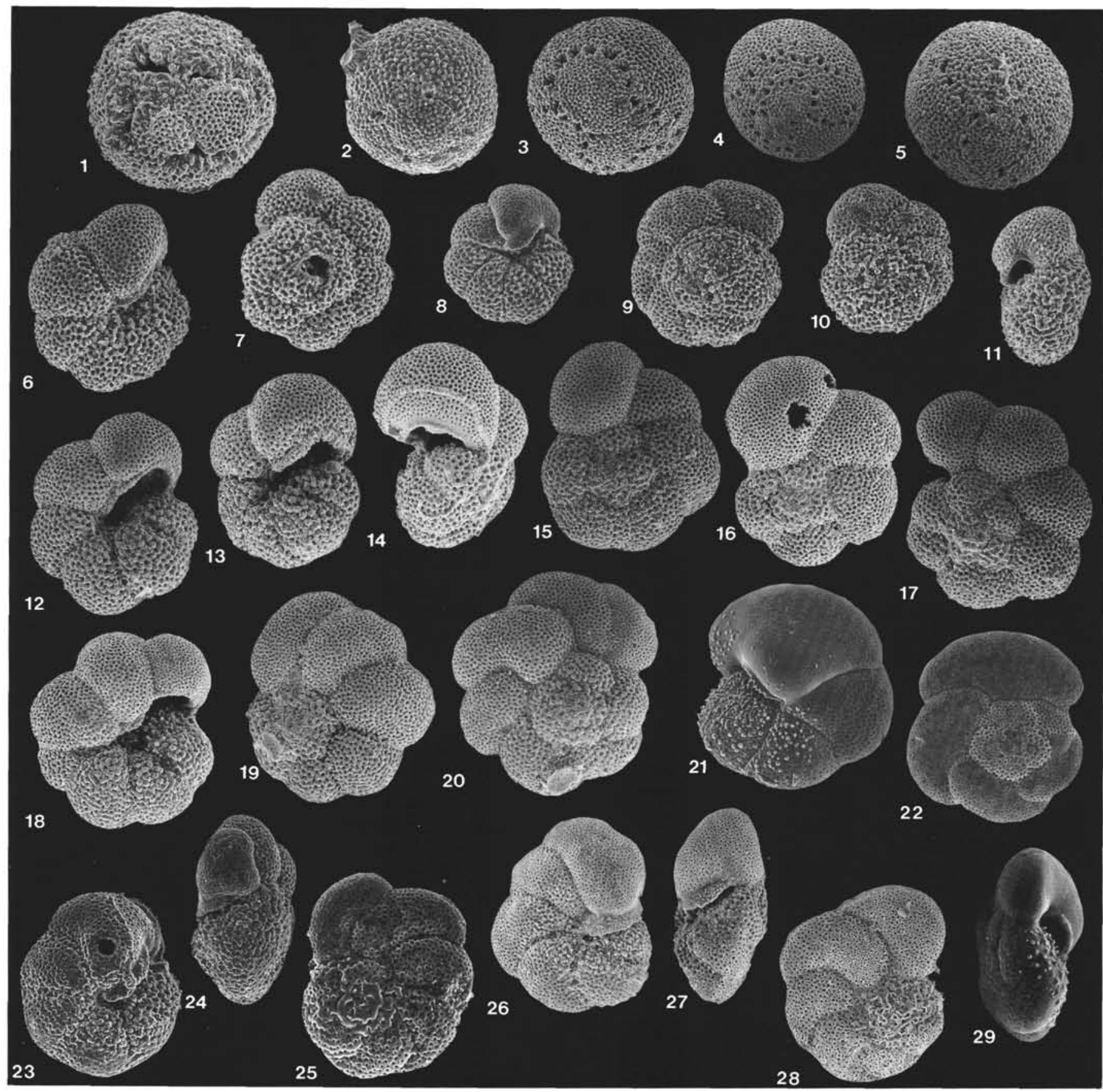

Plate 3. 1, 2. Praeorbulina glomerosa glomerosa; (1) Sample 115-714A-13H-5, 140-145 cm, $\times 60$; (2) Sample 115-714A-13H-4, 20-25 cm, $\times 60$. 3. Praeorbulina glomerosa circularis; Sample 115-714A-13H-5, 140-145 cm, $\times 60$. 4, 5. Orbulina suturalis; (4) Sample 115-714A-10H-CC, $\times 52$; (5) Sample 115-714A-12H-4, 105-110 cm, $\times 60$. 6, 7. Globorotalia pseudokugleri; Sample 115-707A-15X-CC, $\times 90$. 8-11. Globorotalia kugleri; Sample 115-707A-15X-CC, $\times 90$. 12-20. Globorotalia mayeri-G. siakensis group; $(12-17,20)$ Sample $115-707 \mathrm{~A}-13 \mathrm{H}-\mathrm{CC} ;(12,13,16,17) \times 75 ;(14$, $15) \times 67 ;(18,19)$ Sample 115-714A-12H-3, 140-145 cm; $(18) \times 60 ;(19) \times 67.21,22,29$. Globorotalia scitula; $(21)$ Sample $115-705 \mathrm{~A}-1 \mathrm{H}-\mathrm{CC}, \times 60$; (22) Sample 115-716B-28H-CC, $\times 75$; (29) Sample 115-705A-2H-4, 80-82 cm, $\times 75$. 23-25. Globorotalia peripheroronda; (23, 24) Sample 115707A-14H-CC, $\times 75$; (25) Sample 115-714A-13H-5, 20-25 cm, $\times 82$. 26-28. Globorotalia peripheroacuta; (26) Sample 115-714A-13H-4, 20-25 cm, $\times 75 ;(27,28)$ Sample $115-714$ A-12H-3, 140-145 cm; (27) $\times 82 ;(28) \times 75$. 


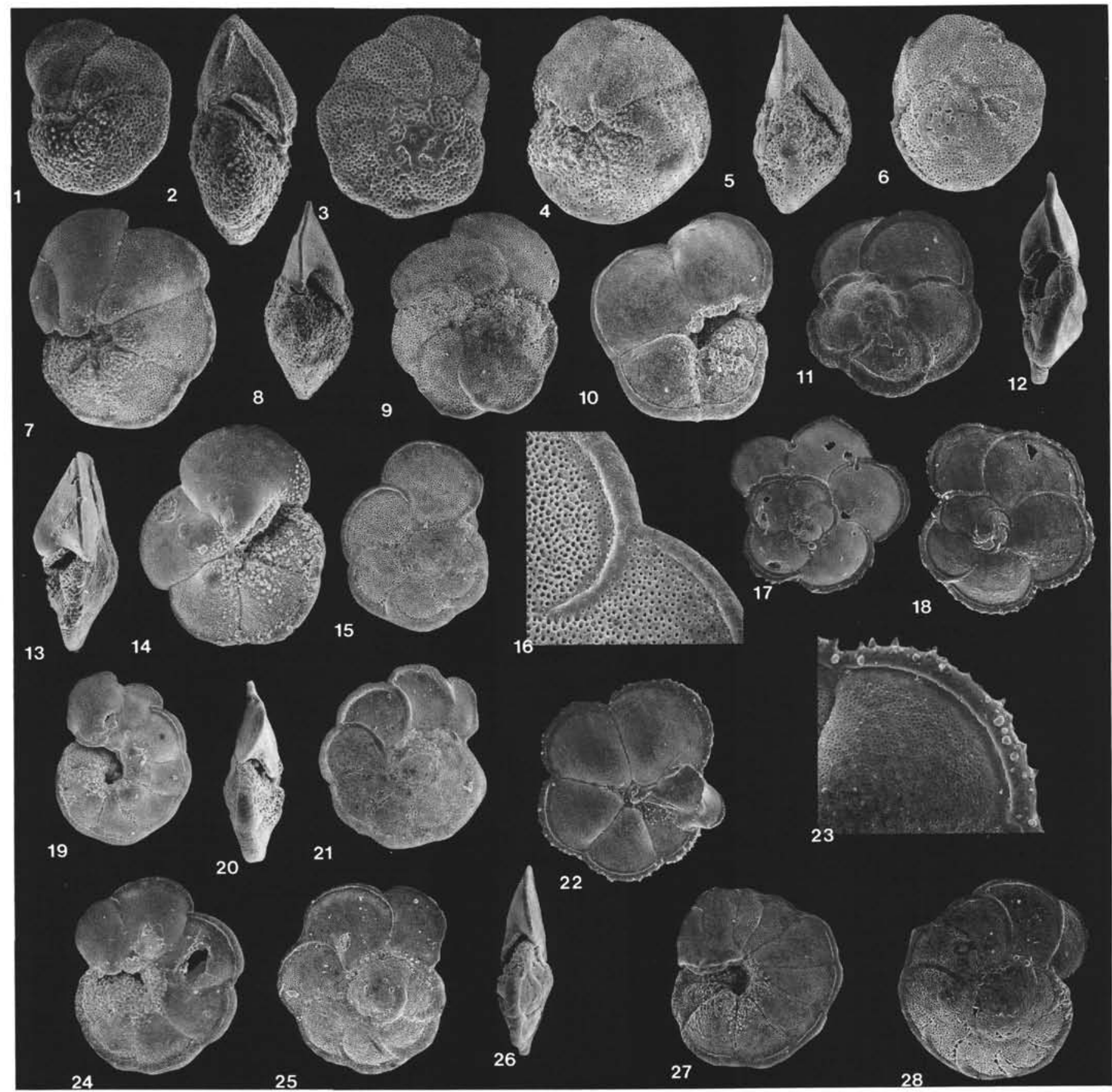

Plate 4. 1-3. Globorotalia praefohsi; (1) Sample 115-714A-11H-5, 20-25 cm, $\times 75$; (2) Sample 115-714A-11H-4, 20-25 cm, $\times 75$; (3) Sample 115714A-11H-6, 20-25 cm, $\times 75$. 4-6. Globorotalia fohsi fohsi; Sample 115-707A-13H-CC; (4) $\times 75 ;(5) \times 67 ;(6) \times 60$. 7-9. Globorotalia fohsi lobata; Sample 115-707A-13H-CC; $(7,9) \times 52 ;(8) \times 60$. 10-18, 22, 23. Globorotalia menardii s.1.; (10-12) Sample 115-706A-1H-1, 80-83 cm, $\times 27$; $(13,14)$ Sample 115-707A-13H-3, 107-112 cm, $\times 52 ;(15,16)$ Sample 115-707A-9H-5, 107-112 cm; $(15) \times 37 ;(16) \times 112 ;(17,18,22,23)$ G. menardii var. fimbriata, Sample 115-714A-1H-CC; $(17,18,22) \times 27 ;(23) \times 90$. 19-21, 24-28. Globorotalia limbata; $(19-21)$ Sample 115-707A-5H-2, 107$112 \mathrm{~cm}, \times 27 ;(24,25)$ Sample 115-707A-9H-5, 107-112 cm, $\times 37$; (26) Sample 115-707A-11H-CC, $\times 31 ;(27,28)$ Sample 115-705A-2H-5, 80-82 cm, $\times 45$. 


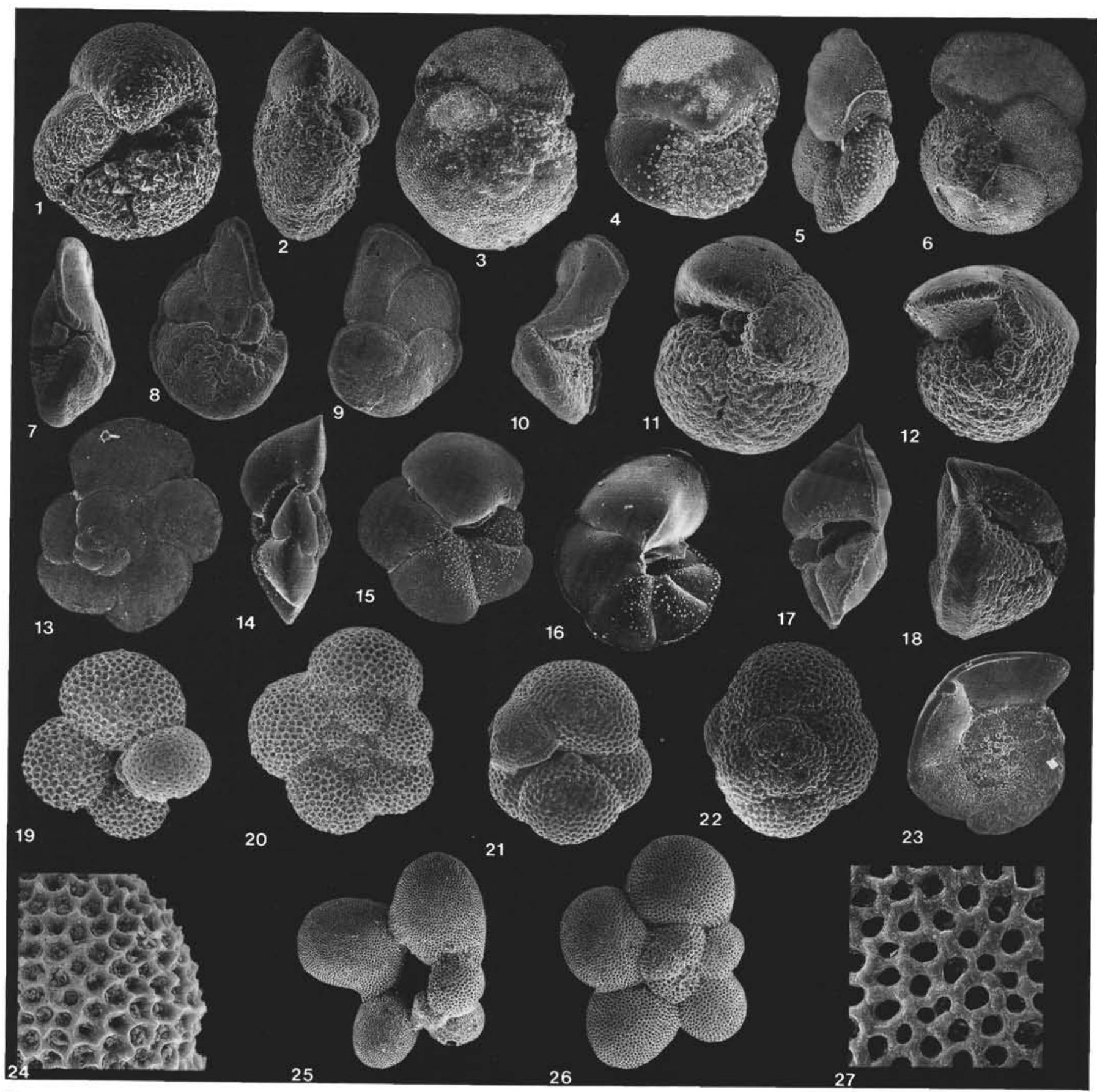

Plate 5. 1-3. Globorotalia miozea; Sample 115-707A-13H-CC; $(1,2) \times 60 ;(3) \times 56$. 4-6. Globorotalia margaritae; Sample $115-707 \mathrm{~A}-5 \mathrm{H}-\mathrm{CC} ;(4)$ $\times 75 ;(5) \times 67 ;(6) \times 60$. 7. Globorotalia tumida tumida; Sample $115-706 \mathrm{~A}-1 \mathrm{H}-1,80-83 \mathrm{~cm}, \times 27.8-10$. Globorotalia tumida flexuosa; Sample 115-709C-5H-1, 60-65 cm, $\times 27$. 11. Globorotalia crassaformis; Sample $115-707$ A-2H-CC, $\times 52$. 12, 18, 23. Globorotalia truncatulinoides; Sample 115-706A-1H-2, 73-76 cm; $(12,18) \times 45 ;(23) \times 38$. 13-15. Globorotalia theyeri; Sample 115-715A-1R-CC; $(13) \times 45 ;(14,15) \times 52$. 16, 17. Globorotalia ungulata; Sample 115-706A-1H-1, 80-83 cm, $\times 40$. 19, 20, 24. Globorotaloides hexagona; Sample 115-707A-5H-CC; (19) $\times 75 ;(20)$ $\times 90 ;(24) \times 373$. 21, 22. Globorotaloides suteri; Sample 115-707A-14H-CC; $(21) \times 75 ;(22) \times 60$. 25-27. Globorotaloides variabilis; Sample 115 $714 \mathrm{~A}-6 \mathrm{H}-\mathrm{CC} ;(25) \times 45 ;(26) \times 49 ;(27) \times 373$. 


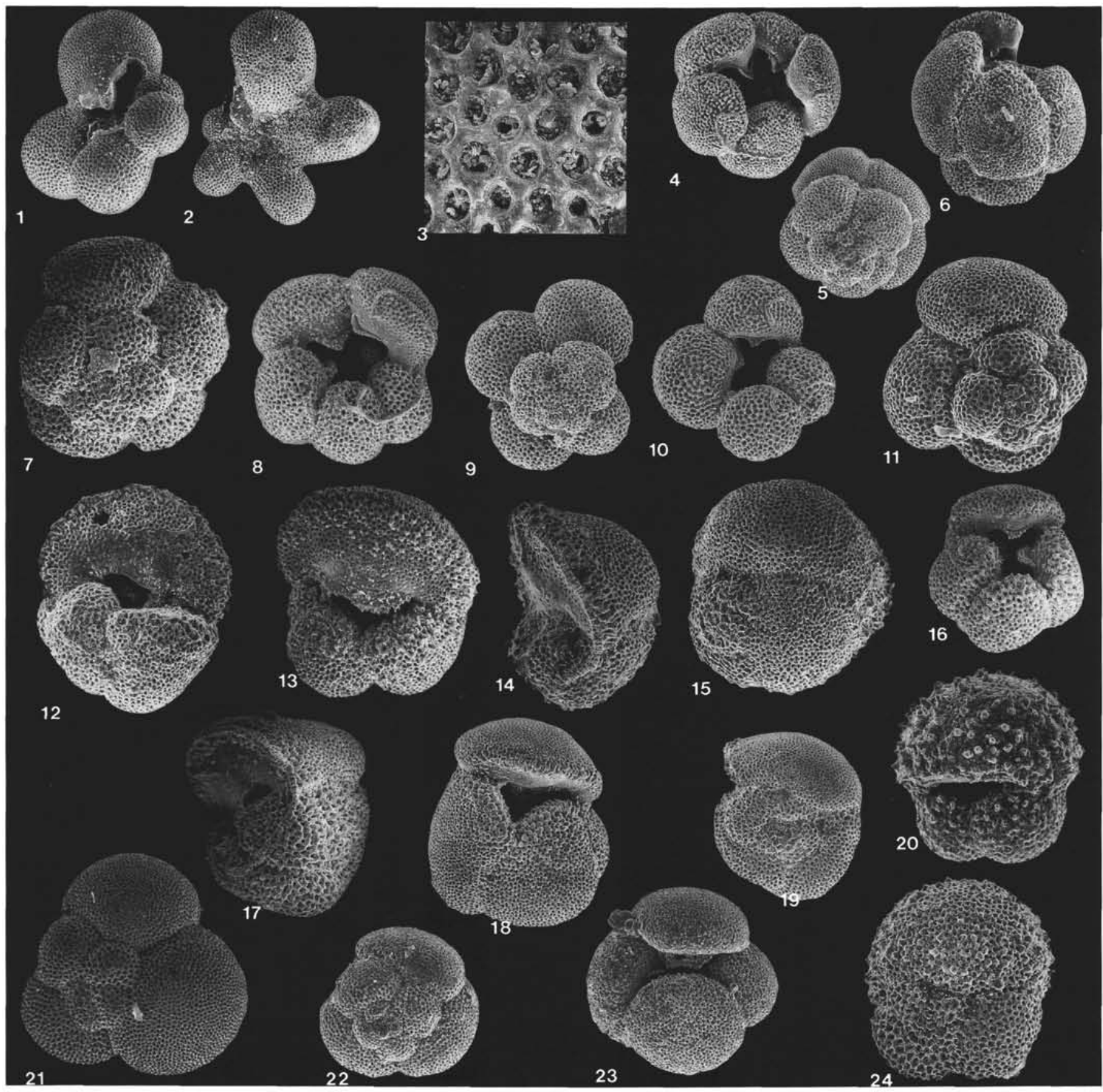

Plate 6. 1-3. Clavatorella bermudezi; $(1,3)$ Sample $115-709 \mathrm{C}-14 \mathrm{H}-5,120-125 \mathrm{~cm} ;(1) \times 45 ;(3) \times 373$; (2) Sample $115-714 \mathrm{~A}-12 \mathrm{R}-6,140-145 \mathrm{~cm}$, $\times$ 49. 4-6. Dentoglobigerina altispira altispira; Sample $115-707 \mathrm{~A}-6 \mathrm{H}-5,107-112 \mathrm{~cm}, \times 45.7,8$. Dentoglobigerina altispira globosa; Sample $115-$ 707A-13H-CC; (7) $\times 56$; (8) $\times 60$. 9, 10. Dentoglobigerina globularis; Sample 115-707A-15H-5, 107-112 cm, $\times 52$. 11, 16. Globoquadrina baroemoenensis; (11) Sample 115-714A-19X-CC, $\times 60$; (16) Sample 115-707A-13H-CC, $\times 60 . \quad 12-15$. Globoquadrina binaiensis; (12, 14, 15) Sample 115714A-19X-CC; (12) $\times 60$; (14) $\times 67$; (15) $\times 60$; (13) Sample 115-707A-13H-CC, $\times 60$. 17. Globoquadrina dehiscens; Sample 115-707A-13H-CC, $\times 67$. 18, 19. Globoquadrina praedehiscens; Sample 115-709C-7H-CC, $\times 52 . \quad 20,24$. Globoquadrina sellii; Sample $115-709 \mathrm{C}-23 \mathrm{X}-5,105-110 \mathrm{~cm}$; (20) $\times 52$; (24) $\times 56$. 21. Globoquadrina conglomerata; Sample $115-705$ A-2H-6, 36-38 cm, $\times 45$. 22, 23. Globoquadrina venezuelana; Sample $115-707 \mathrm{~A}-15 \mathrm{H}-3,107-112 \mathrm{~cm}, \times 45$. 


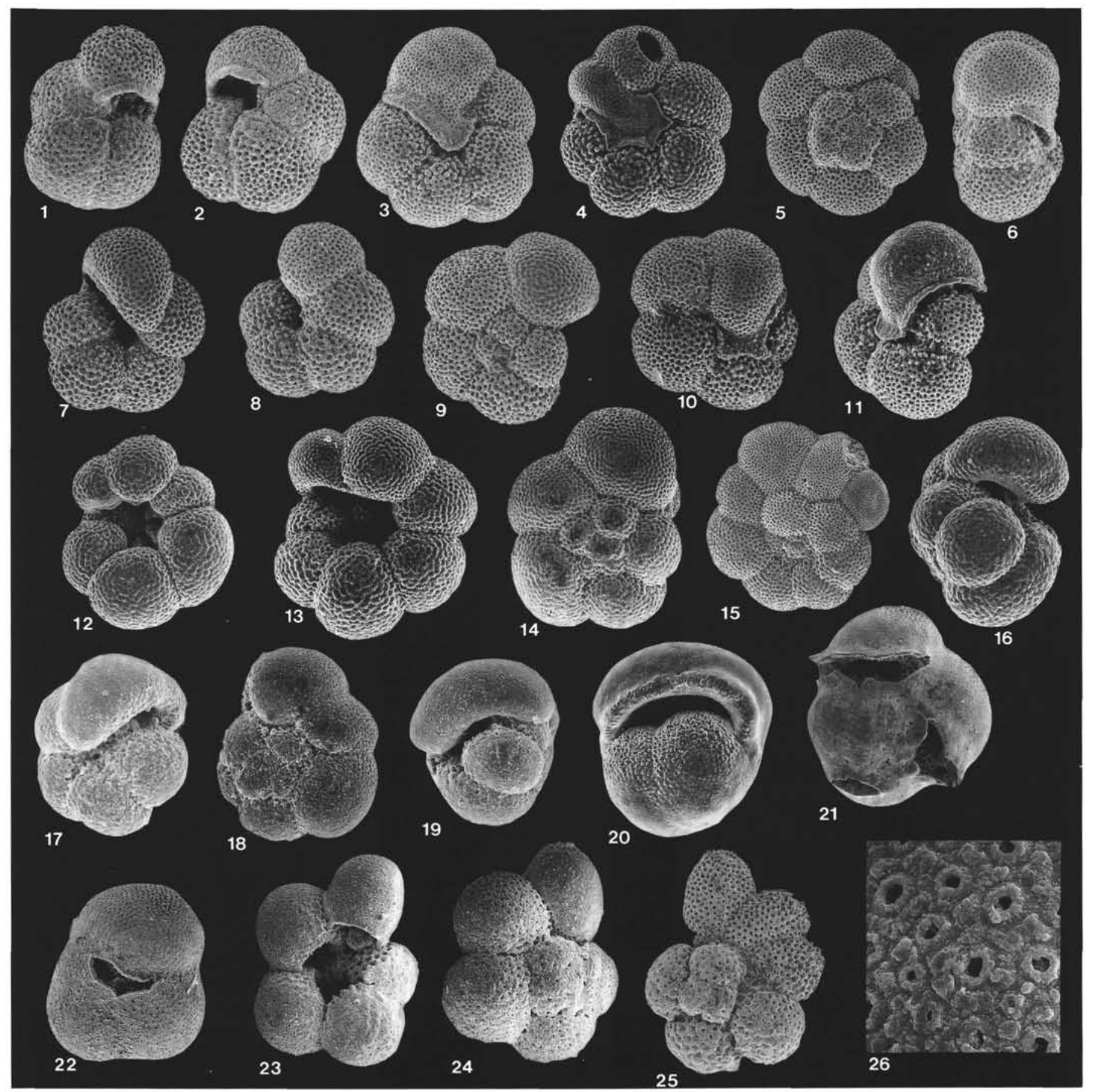

Plate 7. 1, 2. Globoturborotalita nepenthes; Sample 115-707A-5H-CC, $\times 75$. 3-6, 10, 11. Neogloboquadrina acostaensis; (3-5) Sample 115707A-9H-CC, $\times 75$; (6) Sample 115-707A-9H-6, 107-112 cm, $\times 75$; $(10,11)$ Sample $115-714 \mathrm{~A}-4 \mathrm{H}-3,20-25 \mathrm{~cm} ;(10) \times 90$; (11) $\times 97$. 7-9. Neogloboquadrina humerosa; Sample 115-707A-5H-CC, $\times 75$. 12-16. Neogloboquadrina dutertrei; $(12)$ Sample $115-706 \mathrm{~A}-1 \mathrm{H}-\mathrm{CC}, \times 52 ;(13,14)$ Sample 115-707A-2H-5, 107-112 cm; (13) $\times 60$; $(14) \times 56$; $(15,16)$ Sample 115-706A-2H-1, 80-83 cm; $(15) \times 52 ;(16) \times 60$. 17-19. Pulleniatina primalis; Sample 115-707A-8H-2, 107-112 cm, $\times 75$. 20. Pulleniatina obliquiloculata; Sample $115-707 \mathrm{~A}-3 \mathrm{H}-1,107-112 \mathrm{~cm}, \times 52$. 21, 22. Sphaeroidinella dehiscens; (21) Sample 115-706A-1H-2, 73-76 cm, $\times 31$; (22) Sample 115-707A-5H-2, 105-112 cm, $\times 37 . \quad 23-26$. Sphaeroidinellopsis kochi; (23, 24, 26) Sample 115-707A-13H-CC; $(23,24) \times 49$; (26) $\times 373$; (25) Sample 115-707A-11H-CC, $\times 31$. 